\title{
Investigation of Terfenol-D for Magnetostrictive Tagging of Fiber-Reinforced Polymer Composites
}

Robert F. Quattrone, Justin B. Berman, Jonathan C.

December 2000

Trovillion, Carl A. Feickert, Jason M. Kamphaus,

Scott R. White, Victor Giurgiutiu, and Gordon L. Cohen

US Army Corps of Engineers

Engineer Research and Development Center
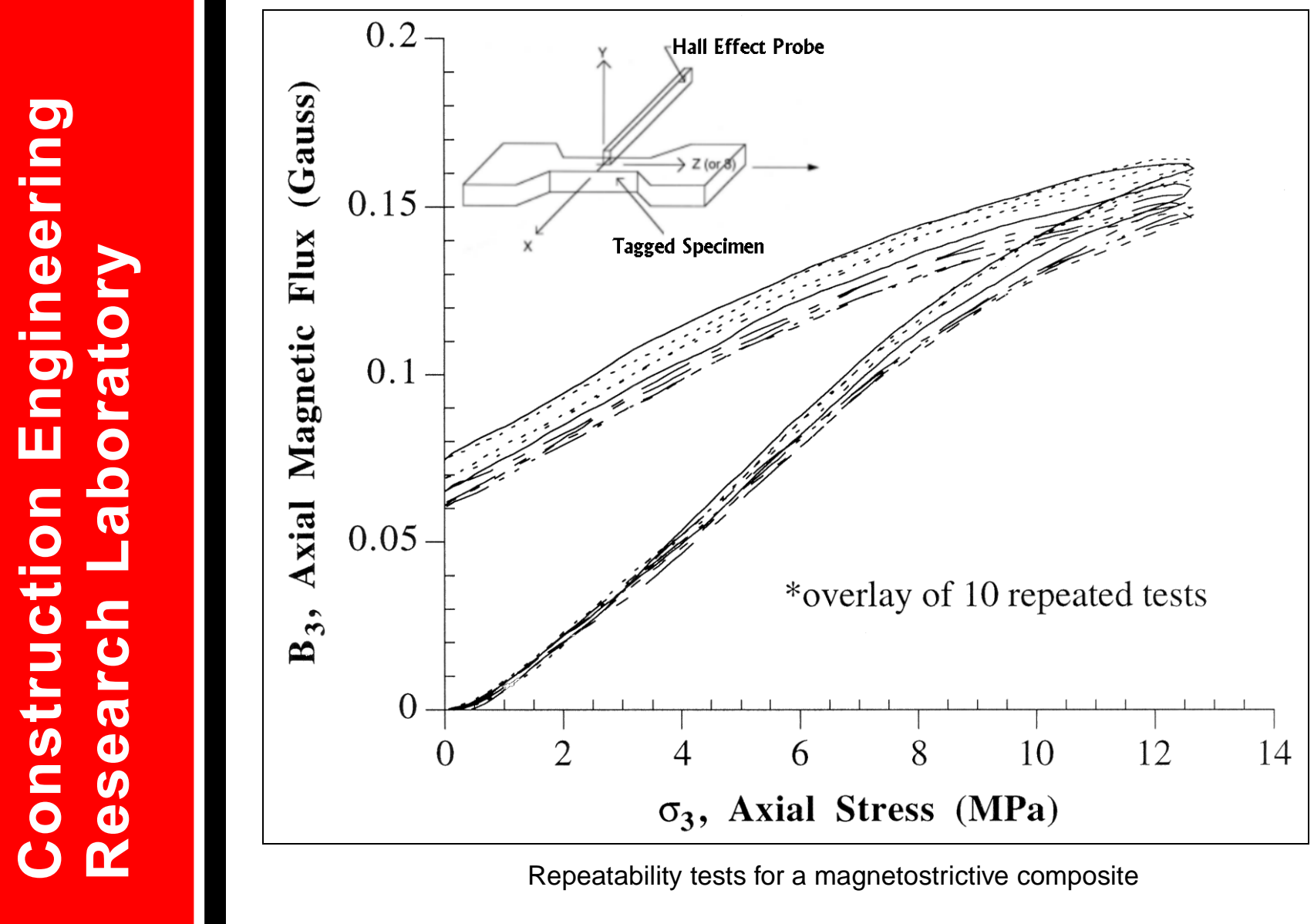

Repeatability tests for a magnetostrictive composite 


\section{Foreword}

This research was conducted for the Directorate of Military Programs, Headquarters, U.S. Army Corps of Engineers, under Project 601102AT23, "Basic Research/Military Construction"; Work Unit FM-E00, "Magnetostrictive Structural Materials." The technical monitor was Charles Gutberlet, J r., CEMP-ED.

The work was performed by the Materials and Structures Branch (CF-M) of the Facilities Division (CF), Construction Engineering Research Laboratory (CERL). The Principal Investigator was Dr. Robert F. Quattrone. Mark W. Slaughter is Acting Chief, CEERD-CF-M, and L. Michael Golish is Chief, CEERD-CF. The Acting Technical Director of the Facility Acquisition and Revitalization Business Area is Dr. Paul A. Howdyshell. The Acting Director of CERL is William D. Goran.

A portion of this work was performed under contract by Dr. Scott White, University of Illinois at Urbana-Champaign, and Dr. Victor Giurgiutiu, University of South Carolina. Matching funds and manufacturing of some composite samples were provided by Reichhold Chemicals, Inc., Research Triangle Park, NC, under a Cooperative Research and Development Agreement (CRDA) with the Market Development Alliance of the Composites Institute.

CERL is an element of the U.S. Army Engineer Research and Development Center (ERDC), U.S. Army Corps of Engineers. The Director of ERDC is Dr. J ames R. Houston and the Commander is COL J ames S. Weller.

\section{DISCLAIMER}

The contents of this report are not to be used for advertising, publication, or promotional purposes. Citation of trade names does not constitute an official endorsement or approval of the use of such commercial products. All product names and trademarks cited are the property of their respective owners.

The findings of this report are not to be construed as an official Department of the Army position unless so designated by other authorized documents.

DESTROY THIS REPORT WHEN IT IS NO LONGER NEEDED. DO NOT RETURN IT TO THE ORIGINATOR. 


\section{Contents}

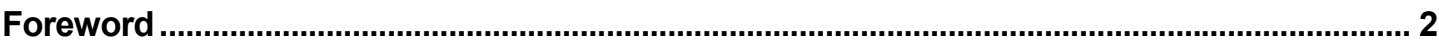

List of Figures and Tables............................................................................................... 5

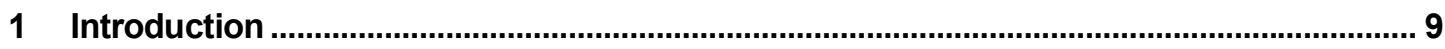

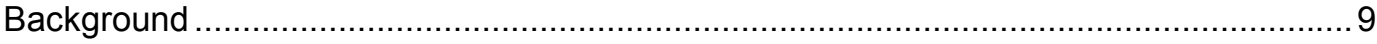

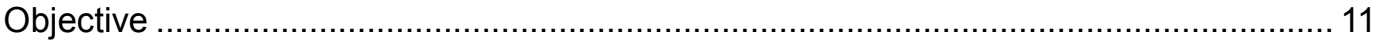

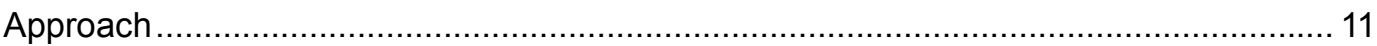

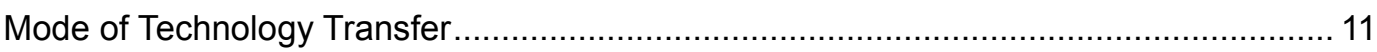

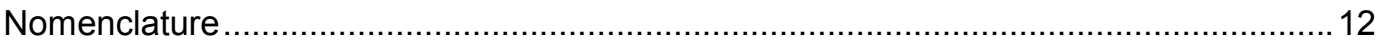

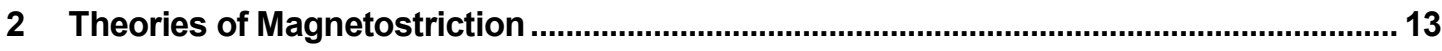

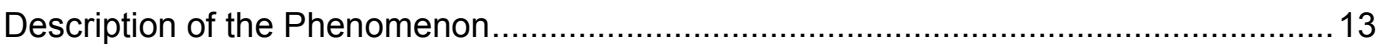

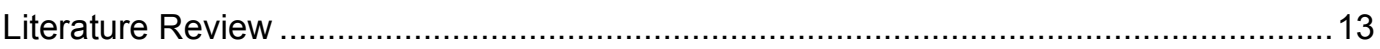

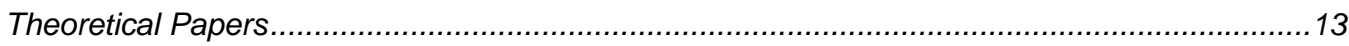

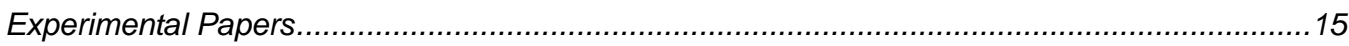

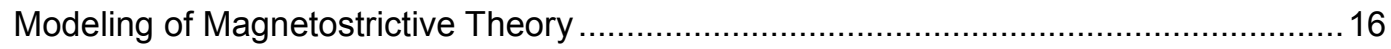

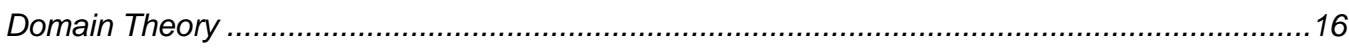

Phenomenological Approach: A Modification of Heisenberg-Weiss Theory ..............................18

Mechanistic Approach: Magnetoelasticity Theory ………...............................................25

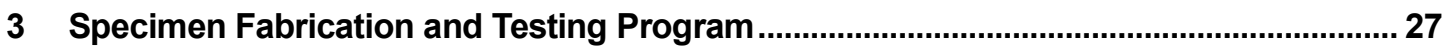

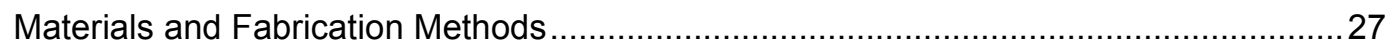

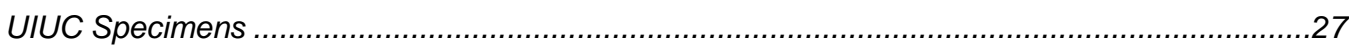

CERL Specimens

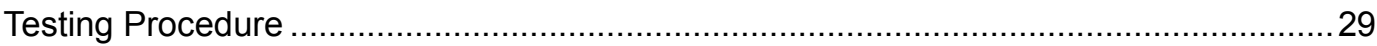

4 Tension Testing of Tagged Neat Resin Specimens........................................................ 31

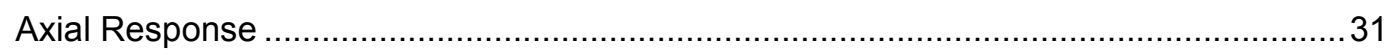

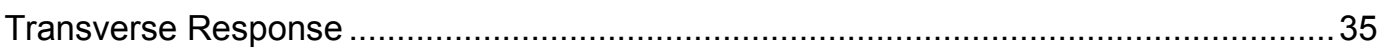

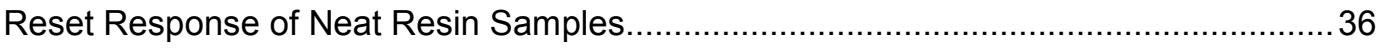

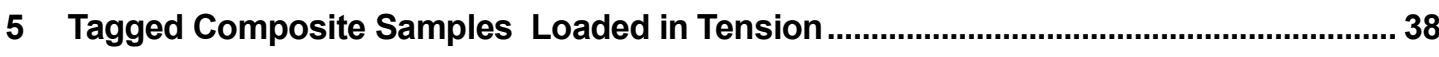

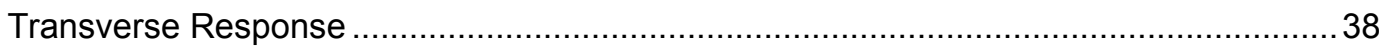

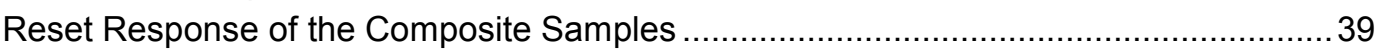

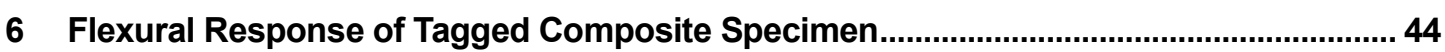

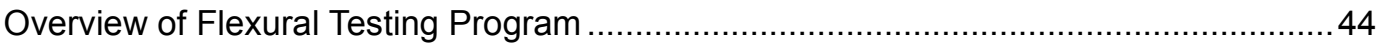

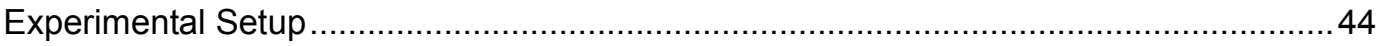

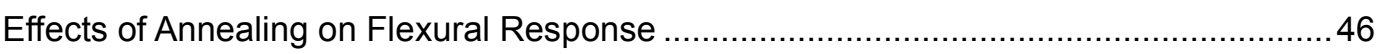

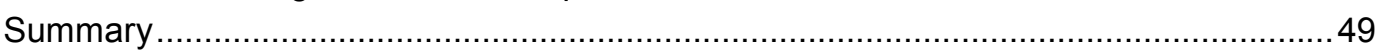


7 Damage Detection in Magnetostrictive-Tagged Composites ........................................... 50

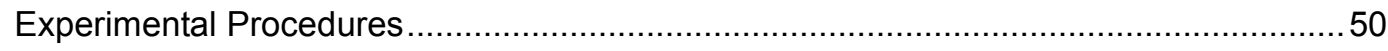

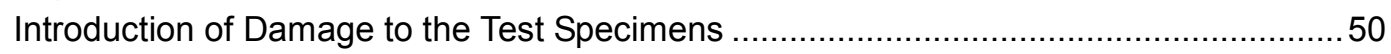

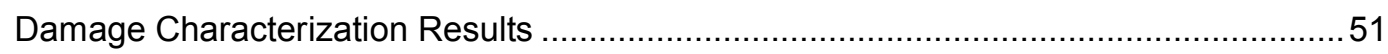

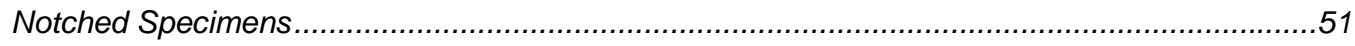

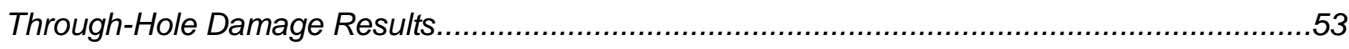

8 Fabrication and Testing of Sputtered Composite Samples................................................64

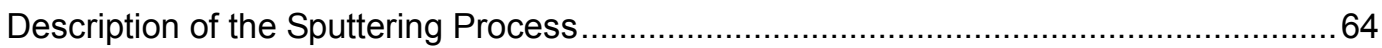

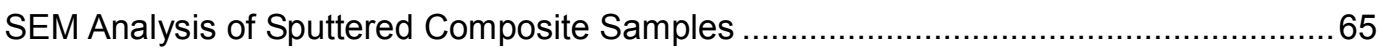

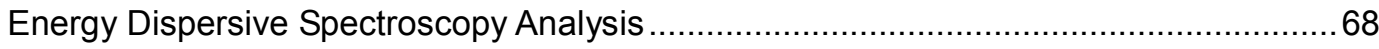

9 Study of Magnetic Domain Structure in Terfenol-D Particles............................................. 70

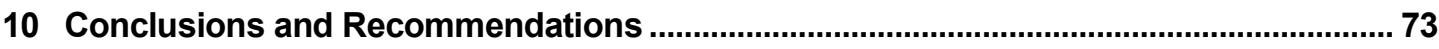

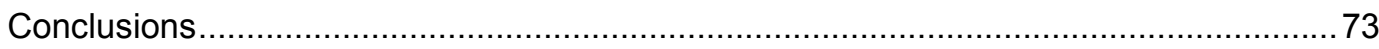

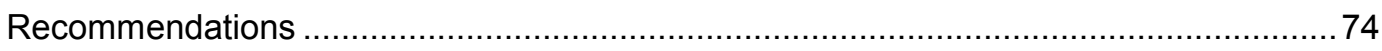

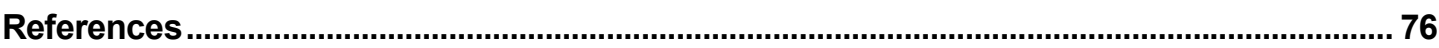

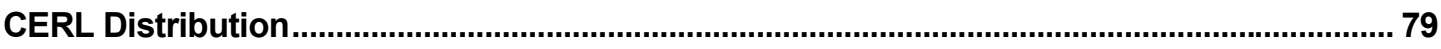

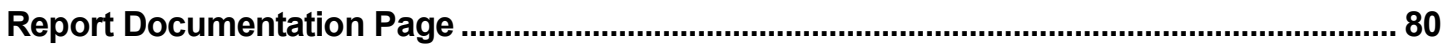




\section{List of Figures and Tables}

\section{Figures}

1 Illustration of domain wall motion and rotation.

2 Comparison of the normalized experimental data of Trovillion et al. (1999) to the three-parameter least squares fit of Equation (2) cast in the form of $B=$ $B_{o} \tanh \left(\alpha B / B_{o}+\chi(F / k N)\right)$, and using the values of $\alpha, \gamma$, and $B_{o}$, found in Table 3. .....24

3 Silicone rubber mold and permanent magnets used during specimen

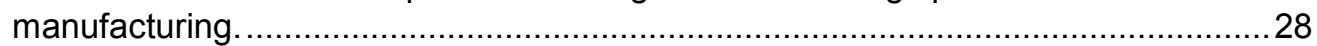

4 Description of coordinate system used for experiments. ......................................29

5 Repeatability tests for a magnetostrictive composite. ......................................... 32

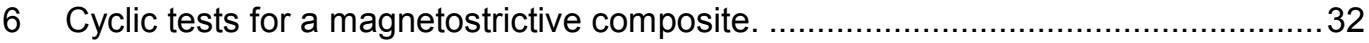

7 Effect of magnetic annealing field strength on sensitivity...................................... 33

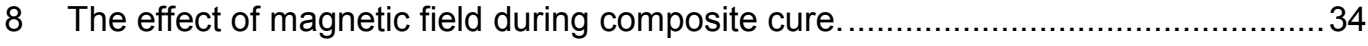

9 Magnetostrictive composite piezomagnetic coefficient $d_{33}$ vs volume fraction. .........34

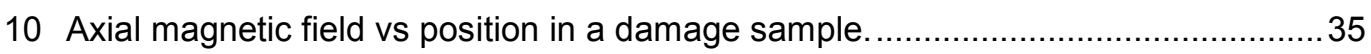

11 Transverse (x-axis) magnetic flux density vs load, neat resin samples. ....................35

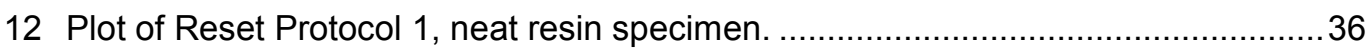

13 Plot of Reset Protocol 2, neat resin specimen. .................................................. 36

14 Summary plot for Reset Protocol 1, neat resin specimen. ....................................... 37

15 Summary plot for Reset Protocol 2, neat resin specimen. ....................................37

16 Typical zorro curve for chained composite specimen with annealing. ......................38

17 Typical zorro curve for unchained composite specimen with annealing. ...................39

18 Plot of Reset Protocol 1, chained composite specimen. .......................................40

19 Plot of Reset Protocol 2, chained composite specimen.........................................40

20 Plot of Reset Protocol 1, unchained composite specimen..................................... 41

21 Plot of Reset Protocol 2, unchained composite specimen.....................................41

22 Summary plot for Reset Protocol 1, chained composite specimen..........................42

23 Summary plot for Reset Protocol 2, chained composite specimen..........................42

24 Summary plot for Reset Protocol 1, unchained composite specimen........................43

25 Summary plot for Reset Protocol 2, unchained composite specimen.......................43

26 Gaussmeter probe, strain gage, and displacement transducer on the magnetostrictive composite beam. 
27 Magnetic field vs applied force for specimen without annealing between cycles: (a) original data for 10 loading cycles; (b) mean value and standard deviation.

28 Magnetic field vs strain for specimen without annealing between cycles: (a) original data for 10 loading cycles; (b) mean value and standard deviation.

29 Magnetic field vs force for specimen with annealing between cycles: (a) original data for 10 loading cycles; (b) mean value and standard deviation.

30 Magnetic field vs strain for specimen with annealing between cycles: (a) original data for 10 loading cycles; (b) mean value and standard deviation.

31 Geometry of damage specimens.

32 Change in magnetic flux in the chain direction at $\sigma_{1}=24 \mathrm{MPa}$ for notched terfenol-D FRP specimens with notch tip positioned at $\mathrm{x}_{2}=-6.4 \mathrm{~mm}$.

33 Difference between damaged (notched) and undamaged $\Delta \mathrm{B}_{2}$ readings for terfenol-D FRP specimens at $\sigma_{1}=24 \mathrm{MPa}$ with notch tip positioned at $\mathrm{x}_{2}=-6.4$ $\mathrm{mm}$.

34 Change in magnetic flux $\Delta \mathrm{B}_{2} \sigma_{1}=24 \mathrm{MPa}$ for notched terfenol-D FRP specimens with notch tip positioned at $\mathrm{x}_{2}=-6.4 \mathrm{~mm}$.

35 Difference between damaged (notched) and undamaged $\Delta \mathrm{B}_{2}$ readings for terfenol-D FRP specimens at $\sigma_{1}=24 \mathrm{MPa}$ with notch tip positioned at $\mathrm{x}_{2}=-6.4$ $\mathrm{mm}$.

36 Difference between damaged (notched) and undamaged $\Delta B_{3}$ readings for terfenol-D FRP specimens at $\sigma_{1}=24 \mathrm{MPa}$ with notch tip positioned at $\mathrm{x}_{2}=-6.4$ $\mathrm{mm}$.

37 Difference between damaged (notched) and undamaged $\Delta \mathrm{B}_{3}$ readings for terfenol-D FRP specimens at $\sigma_{1}=24 \mathrm{MPa}$ with notch tip positioned at $\mathrm{x}_{2}=-6.4$ $\mathrm{mm}$.

38 Difference between damaged (notched) and undamaged $\Delta B_{2}$ readings for terfenol-D FRP specimens at $\sigma_{1}=24 \mathrm{MPa}$ with notch tip positioned at $\mathrm{x}_{2}=-3.2$ $\mathrm{mm}$.

39 Difference between damaged (notched) and undamaged $\Delta \mathrm{B}_{2}$ readings for terfenol-D FRP specimens at $\sigma_{1}=24 \mathrm{MPa}$ with notch tip positioned at $\mathrm{x}_{2}=-3.2$ $\mathrm{mm}$.

40 Difference between damaged (notched) and undamaged $\Delta \mathrm{B}_{3}$ readings for terfenol-D FRP specimens at $\sigma_{1}=24 \mathrm{MPa}$ with notch tip positioned at $\mathrm{x}_{2}=-3.2$ $\mathrm{mm}$.

41 Difference between damaged (notched) and undamaged $\Delta \mathrm{B}_{3}$ readings for terfenol-D FRP specimens at $\sigma_{1}=24 \mathrm{MPa}$ with notch tip positioned at $\mathrm{x}_{2}=-3.2$ $\mathrm{mm}$.

42 Difference between damaged (hole) and undamaged $\Delta \mathrm{B}_{2}$ readings for terfenolD FRP specimens at $\sigma_{1}=24 \mathrm{MPa}$ with hole edges positioned at $\mathrm{x}_{2}=+1.6 \mathrm{~mm}$ and $-1.6 \mathrm{~mm}$.

43 Difference between damaged (hole) and undamaged $\Delta \mathrm{B}_{2}$ readings for terfenolD FRP specimens at $\sigma_{1}=24 \mathrm{MPa}$ with hole edges positioned at $\mathrm{x}_{2}=+1.6 \mathrm{~mm}$ and $-1.6 \mathrm{~mm}$.

44 Difference between damaged (hole) and undamaged $\Delta \mathrm{B}_{3}$ readings for terfenolD FRP specimens at $\sigma_{1}=24 \mathrm{MPa}$ with hole edges positioned at $\mathrm{x}_{2}=+1.6 \mathrm{~mm}$ and $-1.6 \mathrm{~mm}$. 
45 Difference between damaged (hole) and undamaged $\Delta \mathrm{B}_{3}$ readings for terfenolD FRP specimens at $\sigma_{1}=24 \mathrm{MPa}$ with hole edges positioned at $\mathrm{x}_{2}=+1.6 \mathrm{~mm}$ and $-1.6 \mathrm{~mm}$.

46 Difference between damaged (hole) and undamaged $\Delta \mathrm{B}_{3}$ readings for terfenolD FRP specimens at $\sigma_{1}=24 \mathrm{MPa}$ with hole edges positioned at $\mathrm{x}_{2}=+2.4 \mathrm{~mm}$ and $-2.4 \mathrm{~mm}$.

47 Difference between damaged (hole) and undamaged $\Delta \mathrm{B}_{3}$ readings for terfenolD FRP specimens at $\sigma_{1}=24 \mathrm{MPa}$ with hole edges positioned at $\mathrm{x}_{2}=+2.4 \mathrm{~mm}$ and $-2.4 \mathrm{~mm}$.

48 Difference between damaged (hole) and undamaged $\Delta \mathrm{B}_{3}$ readings for terfenolD FRP specimens at $\sigma_{1}=24 \mathrm{MPa}$ with hole edges positioned at $\mathrm{x}_{2}=+3.2 \mathrm{~mm}$ and $-3.2 \mathrm{~mm}$.

49 Difference between damaged (hole) and undamaged $\Delta \mathrm{B}_{2}$ readings for terfenolD FRP specimens at $\sigma_{1}=24 \mathrm{MPa}$ with hole edges positioned at $\mathrm{x}_{2}=+3.2 \mathrm{~mm}$ and $-3.2 \mathrm{~mm}$.

50 SEM image of terfenol-D powder particles, showing coalescence of individual particles and non-uniform distribution (magnification: 256X).

51 SEM image of sputtered terfenol-D on satin fiber weave, showing uniform distribution on the individual glass fibers throughout the thickness (magnification: 378X).

52 Loading and unloading of annealed sputtered unidirectional sample.

53 Loading and unloading of annealed sputtered weave sample.

54 MFM topography and phase images of embedded terfenol-D particles from hammer-milled powder.

55 Close-up MFM topography and phase images of the 7 micron terfenol-D particle boxed in white in Figure 54.

56 MFM topography and phase images of embedded terfenol-D particles from ball-milled powder.

\section{Tables}

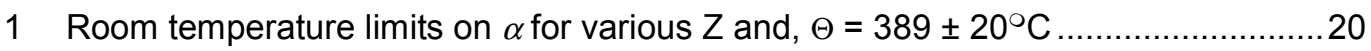

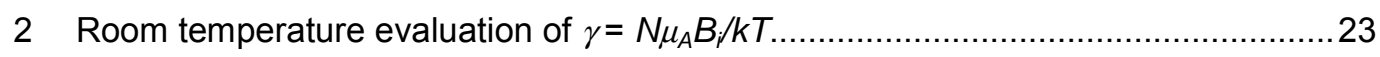

3 Least squares determinations of $\alpha, \gamma$, and $B_{o}$ as defined in Equation (3). All parameters have $\mathrm{a} \pm 4 \%$ error.

4 List of equipment used in flexural testing of tagged composite beam experiment. 



\section{Introduction}

\section{Background}

The U.S. Army maintains an aging inventory of over 143,000 buildings, including more than 2400 barracks and numerous structures of historic significance. These buildings represent $\$ 4.6$ billion in high-priority, unfinanced maintenance and repair $(M \& R)$. Many of these structures fail to meet modern building codes and require structural upgrades. Environmental factors such as seismic motion, high winds, and other natural forces can accelerate structural damage - often at difficult-to-detect locations. Unrepaired structural damage poses the threat of catastrophic failure, interrupt the execution of mission, and create potentially life-threatening conditions for soldiers, their families, and other Army personnel.

Various advanced structural composite materials can provide advantages over conventional structural upgrade systems by offering up to 50 percent first-cost savings and lower life-cycle costs, often along with additional benefits such as easier installation and improved safety. But in spite of the potential benefits of using composites in structural applications, the Army currently avoids using them on a wide scale because there is still a lack of data on the long-term performance of these materials. Fiber-reinforced polymer (FRP) composites typically fail in a brittle and catastrophic manner with little forewarning. Such a stealthy, unforgiving failure process is especially disconcerting to a conservative civil engineering and construction community. Material scientists are working to change the underlying microstructural elements of FRP composites in order to provide a more progressive failure mode. But the most common and costeffective polymer matrices currently available are thermosetting polymers such as polyester, vinyl ester, and epoxy. These polymers cannot be significantly modified in terms of microstructural properties, so composites based on them will al ways be characterized by brittle fracture modes.

Another way to address this failure mode problem is to engineer a composite material with the ability to warn of unusual stresses developing damage, and potential failure. This concept differs from destructive and nondestructive evaluation methods (such as ultrasonic C-scan, x-ray radiography, visual inspection, etc.) in that the material itself - not external manual intervention - provides informa- 
tion on its own condition. A material possessing such a capability would be said to be self-assessing; that is, possessing the ability to assess its own condition. Such a material would fall into a category commonly referred to as smart mate rials, which are materials that are active or reactive based on their environment. A smart composite material with self-assessment capability could address engineering concerns and ultimately enable the wider application of FRP composites to civil works structures. This type of material can be fabricated using the technique called tagging.

Tagging of a composite refers to the use of a third phase in the material, usually an active (smart) material that is incorporated into the matrix. This third phase can comprise particles, wires, ribbons, thin films, or other geometries. Previous research by the Construction Engineering Research Laboratory (CERL) has demonstrated that the tagging material can be piezoelectric (Quattrone et al. 1998; Berman et al. 1999), ferromagnetic (Quattrone et al. 1998), magnetostrictive (Quattrone et al. 1998), or shape memory (Quattrone et al. 1998).

The basic research documented in this report focuses on the tagging of composite materials by incorporating magnetostrictive particles into the polymer matrix during manufacture. Magnetostrictive particles are mixed into the polymer resin before the reinforcement materials are embedded. The particles typically measure 1 - 100 microns in diameter. The most common magnetostrictive material used for this type of application is terfenol-D, an alloy of terbium, dysprosium, and iron having the general chemical formula $\mathrm{Tb}_{x} \mathrm{Dy}_{1-x} \mathrm{Fe}_{2}$ where $\mathrm{x}$ is 0.3. Terfenol-D is a so-called giant magnetostrictive, and it has the largest magnetostrictive constant (at room temperature) of any material. After the terfenol-D particles are mixed into the polymer phase, the composite structure is fabricated and cured while being subjected to an aligning magnetic field. After curing is complete and appropriate annealing treatments have been made, the composite member possesses the ability to produce self-assessment data that can be logged, processed, and interpreted.

Tagging with magnetostrictive particles offers many advantages over other methods and geometries. The particles can be distributed throughout the matrix phase, allowing for the collection of stress data from all portions of a structural element. The magnetostrictive particles also work well with wireless datagathering technologies; data can be acquired using a Hall-effect sensor that does not have to be hard-wired to the composite. 


\section{Objective}

The objective of this research was to develop a cost-effective magnetostrictive FRP composite material that can be fabricated into structural el ements capable of generating data about their own structural condition, and which can be monitored and assessed by measuring its electromagnetic flux over time.

\section{Approach}

The magnetostrictive material selected for these tagging experiments was terfenol-D, which exhibits both direct and indirect magnetostriction phenomena. The current studies focused on composites with a low volume fraction (less than 10 percent) of terfenol-D powder embedded in a polyester (or epoxy) resin or a glass/polyester (or epoxy) composite.

Experimental and theoretical work was conducted to test the validity of magnetostrictive particles as tags. The major tasks, documented in detail in the body of this report, comprised the following:

1. Magnetostrictive particles were embedded in polyester or epoxy samples with and without reinforcing fibers.

2. A test procedure was developed and the specimens were tested according to this procedure.

3. Microscopic observations were made to investigate the correlation between the microscopic and macroscopic features of the material.

4. A semi-quantitative phenomenological model was developed to explain the experimental data.

\section{Mode of Technology Transfer}

A Small Business Innovative Research (SBIR) project has been initiated to create a more responsive sensor that would enable the use of less magnetostrictive tagging material, thereby lowering the cost of this technology. Also, a commercial manufacturer has begun producing composites utilizing this magnetostrictive tagging technology. Both of these private-sector activities will feed into a demonstration opportunity under Project 601102AT41, "Military Facilities Engineering Technology;" Work Unit CF-50, "Seismic Rehabilitation of Unreinforced Masonry Walls." 


\section{Nomenclature}

$B_{i} \quad$ magnetic flux vector

$B_{j}(z)$ Brillouin function

B o maximum magnetic flux

$B_{x}$ external magnetic field

$d_{i j} \quad$ piezomagnetic tensor

$\mathrm{H}_{\mathrm{j}} \quad$ magnetic field

$J_{e} \quad H$ eisenberg exchange integral

$M$ magnetization in a magnetic field

$M_{i j k}$ magnetostrictive tensor

$M_{\circ}$ magnetization in total saturation

$\mathrm{N}$ number of spins

$\mathrm{S}_{\mathrm{ij}}{ }^{\mathrm{H}} \quad$ compliance tensor at constant field

$T_{\text {。 }}$ room temperature

$Y_{\text {mag }}$ magnetostrictive equivalent Young's modulus

$Y_{\text {mech }}$ Young's modulus

Z coordination number

$\Theta \quad$ Curie temperature

$\alpha \quad$ fitting constant derived from Heisenberg-Weiss theory

$\beta \quad$ fitting constant that is a function of various atomic parameters

$\gamma \quad$ fitting constant derived from Heisenberg-Weiss theory

$\varepsilon_{\mathrm{i}} \quad$ reduced strain tensor

$\sigma_{\mathrm{j}}$ reduced stress tensor

$\mu_{\mathrm{A}} \quad$ collective spin magnetic moment

$\mu_{\mathrm{B}} \quad$ Bohr magneton

$\mu_{i j}{ }^{\sigma} \quad$ magnetic permeability tensor at constant stress

$\rho_{\text {Mag }}$ collective magnetic energy density

$\rho_{\text {Mech }}$ mechanical energy density 


\section{Theories of Magnetostriction}

\section{Description of the Phenomenon}

Magnetostriction is defined as the change in any dimension of a magnetic material caused by a change in its magnetic state. The magnetostriction constant $\lambda$ is given as the change in length over a gage length. Dimensional change happens via the realignment of magnetic domains. These domains are small regions in the material in which the atomic dipole moments are all aligned in one direction due to strong interaction fields arising from the neighboring dipoles. In the absence of an external magnetic field, for a single crystal the magnetizations in various domains are randomly oriented. The net magnetization on a macroscopic level, therefore, is zero. When a weak magnetic field is applied, the volumes of the domains in which the original magnetizations are parallel to the applied field grow at the expense of other domains. This phenomenon is called domain wall motion. In the presence of a strong magnetic field, domain rotation the realignment of the magnetizations of the individual domains - can occur. Irreversibility of domain wall movement leads to magnetic hysteresis, a residual electromagnetic charge that remains after the magnetic field is removed.

Broadly speaking, magnetostrictive materials may be divided into two forms based on microstructure: crystalline and amorphous. Crystalline magnetostrictives, consisting mainly of metallics and rare-earths, display relatively large saturation strains, especially the so-called giant magnetostrictive materials. Therefore, the crystalline magnetostrictives are ideally suited for actuating purposes.

\section{Literature Review}

\section{Theoretical Papers}

There is a limited volume of literature devoted to the constitutive modeling and theoretical aspects of magnetostriction compared to the large number of papers dealing with experimental results. Constitutive modeling literature will be discussed first, then experimental literature. 
Bergqvist and Engdahl (1991) extended the classical Preisach model to include the stress dependence of the magnetic field. This paper essentially tries to model the field and stress hysteresis curves for magnetostrictive materials. Comparisons with experimental results for terfenol-D give excellent agreement.

Engdahl and Svensson (1988) and Engdahl and Kvarsnjö (1990) developed a dynamic simulation model for the behavior of terfenol-D rods under magnetic and mechanical excitations. Maxwell's equations, the equations of motion, and the nonlinear stress-strain and field relations comprise the constitutive equations of the model. A one-dimensional analysis was used and it did not account for hysteresis. A commercial software package was used to solve the differential equations. This type of analysis was extended to twodimensions by Kvarnsjö and Engdahl (1991) and combined with a Preisach hysteresis model by Bergqvist and Engdahl (1991). Adly, Mayergoyz, and Bergqvist (1991) presented a generalized Preisach model for terfenol-D.

Benbouzid, Reyne, and Meunier (1993) proposed a nonlinear finite element method for modeling terfenol-D. The equations are the same as those used by Kvarnsjö and Engdahl (1991) but a variational formulation is introduced. Commercial packages were used to solve the finite element problem.

Jiles and Thoelke (1991) extended the rotational model presented earlier by Clark et al. (1986) to include a full three-dimensional case. The extension is based on calculating the free energy minima under different stress and field conditions. The change in the minima with loading in the [112] direction, which is typically the axial direction in terfenol-D rods, is presented in the paper and the critical field strengths were found and compared to experimental data. Clark's paper addresses the special case in which the loading is only in the [111] directions and rotations of the magnetic moments are permitted only in the [110] plane.

Bagdasaryan and Danoyan (1992) derived a solution for a thin, infinite plate with different constraints on one edge and made up of two layers of magnetostrictive materials subjected to a dynamic magnetic field. The mathematical formulation is complicated and only the simplest cases (homogeneity, elastic isotropy, etc.) can be solved. The equations of magnetoelasticity are combined with the Kirchhoff thin plate theory. 


\section{Experimental Papers}

One of the first papers to report on embedding magnetostrictive particles is by Sandlund et al. (1994). Their composite material incorporated terfenol-D powder with a nonmetallic binder in a volume fraction of $70-80$ percent powder. The use of a binder created an insulating layer around particles, greatly reducing eddy current losses at high frequency. The researchers also found that the tensile strength of their composite was about four times greater than that of pure terfenol-D alloy.

Hudson, Busbridge, and Piercy (1998) did a systematic study of coupling factors and elastic modulus of terfenol-D/epoxy composites in five different particle size ranges and three different volume fractions. It was found that particle size (100 - 700 microns) had no effect on coupling factor or modulus. These properties scaled with volume fraction in a predictable manner.

Roberts and others (1995) also fabricated terfenol-D/epoxy composites of high volume fraction (approximately 70 percent). They measured the strain-applied field loops and were able to model the initial (linear) portions fairly well.

Most experimental papers on magnetostrictive actuation deal with using monolithic magnetostrictive rods or discs to produce the actuation. However, Mitrovic, Robert, and Carman (1995) reported the results of some analytical and experimental work involving magnetostrictive composite materials. A nonlinear constitutive relation for magnetostrictive materials was developed that includes coupling between temperature/preload and magnetic field strengths. Mitrovic and colleagues manufactured directional magnetostrictive specimens using epoxy and terfenol-D powder. Chains were induced using permanent magnets with a magnetic field $\mathrm{H}$ of about $100 \mathrm{kA} / \mathrm{m}$. The exact particulate volume fraction was unknown, but it was less than 30 percent. The epoxy was cured for 60 minutes at approximately $149^{\circ} \mathrm{C}$ with the magnetic field perpendicular to the direction of gravity. Specimens were $25 \times 6.35 \mathrm{~mm}$. Some of these specimens were then strain gaged and placed in a magnetic field. Results show a longitudinal strain of nearly 100 microstrain for a field of $\mathrm{H}=0.72 \mathrm{kOe}$. The derived constitutive equations and experimental data showed reasonable correlation.

The concept of tagging with magnetostrictive materials was first introduced by Rogers and associated at the Center for Intelligent Materials, Systems, and Structures (CIMSS) at Virginia Polytechnic Institute and State University (1994). These researchers demonstrated elevated signal levels at locations that corresponded to a delamination site in a terfenol-tagged epoxy composite. They 
have also presented fundamental analysis of signal strength for terfenol tagging in an infinite polymer matrix (1995).

J olly and others (1996) developed an elastomer system with embedded ferrous particles at 30 percent volume fraction. The result is an elastomer material that mimics an electrorheological fluid; that is, the modulus of the elastomer can be changed by the application of a magnetic field.

Pinkerton et al. (1997) investigated a novel magnetostrictive of SmF e/metal composite. These researchers made composites with both $\mathrm{Al}$ and $\mathrm{Fe}$ matrices and 50 percent volume fraction $\mathrm{SmFe}_{2}$. Their primary motivation for developing metal matrix composites with magnetostriction was to increase the fracture toughness compared to monolithic terfenol-D so that machining of parts is much easier to accomplish. Similarly, Giurgiutiu and others (1999) used ferromagnetic particles embedded in a polymer composite to provide increased material capabilities. In their work the particles were detected either passively (eddy current probe) or actively (AC* magnetic excitation). In active mode, the force on the surface of the material was tracked with a force gage and the applied magnetic field was measured with a Hall probe. The real and imaginary components of the transfer function were analyzed to assess the effects of damage intentionally introduced in the form of saw cuts or delaminations in the vicinity of the measurements.

\section{Modeling of Magnetostrictive Theory}

\section{Domain Theory}

At the microscopic level magnetostriction (and magnetic materials in general) is characterized by magnetic regions known as domains. A magnetic domain is a region of the material where there is uniform magnetization. In a magnetic material (e.g., Fe, Ni, Dy, Tb, etc.) that has zero remnant magnetization, these magnetic domains are randomly oriented so that the sum of all their magnetization vectors is zero.

* AC: alternating current. 


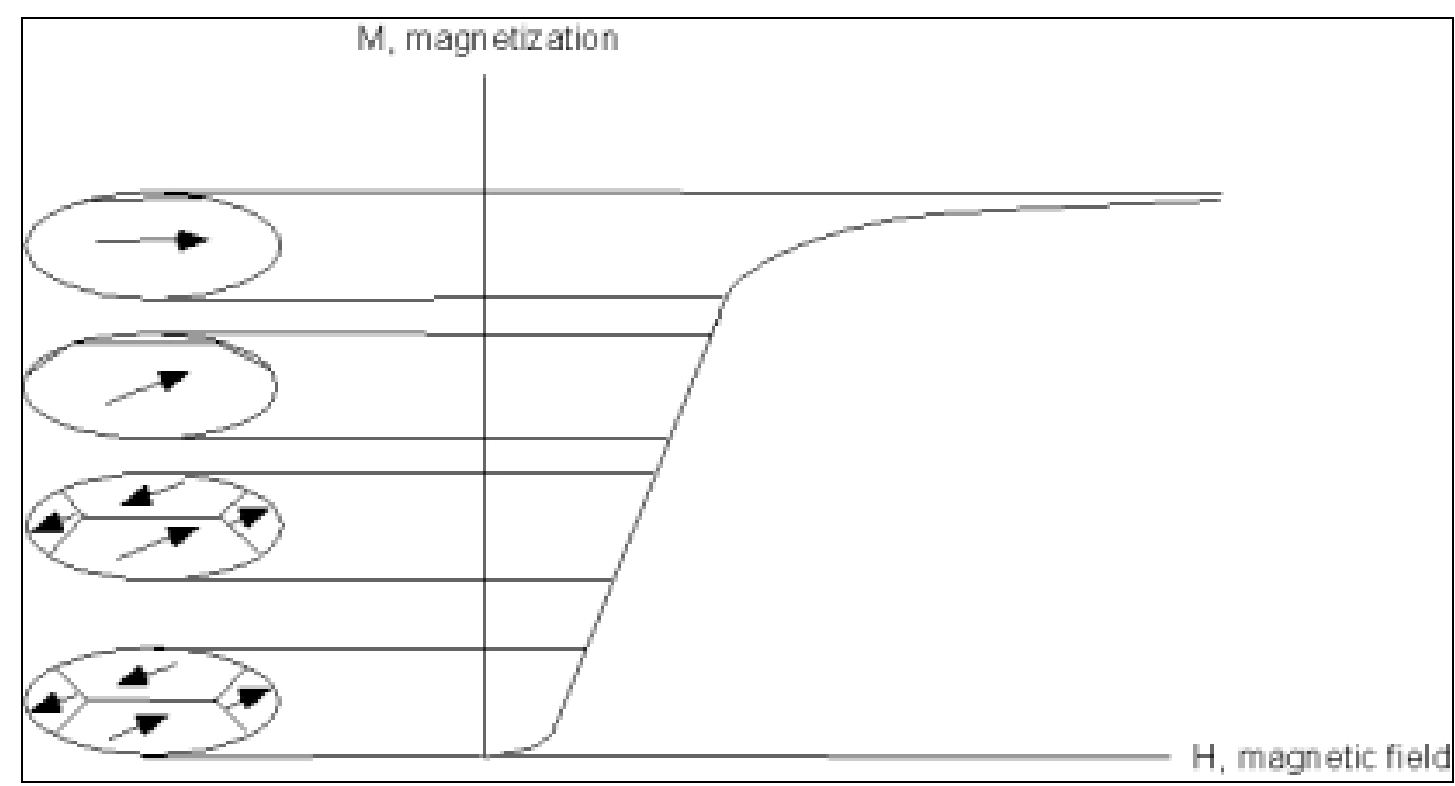

Figure 1. Illustration of domain wall motion and rotation.

The size and orientation of these domains is governed by the energetics of the system. When a magnetic or mechanical stress field is applied to magnetic materials these domains change their size, shape, and orientation of their magnetization in order to minimize the energy. An illustration of these effects when a magnetic field is applied is shown in Figure 1. As the magnetic field is strengthened, some domains grow at the expense of others (first three ovals from bottom). This is known as domain wall motion. When a material with a positive magnetostriction reaches its saturation magnetization, all of the domains form a single domain with an orientation in the direction of the applied field (the top oval). The effect of this phenomenon is that the material physically elongates (du Tremolet de Lacheisserie 1993).

There has been a gap between the theoretical explanation of magnetic domain behavior and the modeling of a composite system that consists of magnetostrictive particles and resin. In order to extend theoretical explanations from the microscopic scale toward a macroscopic model, both a phenomenological (semiquantitative) approach and a mechanistic approach were considered. The current work is based on a semi-quantitative, mesoscale approach; discussion of the mechanistic approach is presented for perspective. 


\section{Phenomenological Approach: A Modification of Heisenberg-Weiss Theory}

The extension of magnetic domain theory from the microscopic to the macroscopic scale can be made through a semi-quantitative phenomenological approach based on the Heisenberg-Weiss Theory (F eickert et al. 2000).

The demonstrated magnetic nature of the terfenol-D resin system strongly suggests an analogy with models for the ferromagnetic system, in particular a model based on the work of Weiss and Heisenberg (H-W). Drawing upon Weiss's initial success (Weiss 1928), Heisenberg was able to use quantum mechanics to refine and quantify the model as it applies to the ferromagnetic system. In doing so, Heisenberg was able to demonstrate that the "molecular field" of Weiss theory was not actually a magnetic field, but expressed the exchange energy stabilizing the parallel spin orientation. An analogous interaction applies to the terfenol system, where the spin moment can be envisioned as rigidly attached, by a strong spin-orbit coupling, to the anisotropically shaped electron charge cloud characteristic of the rare earth elements. A consequence of this "crystal field energy" is the tendency of a magnetic moment to be stabilized in a particular crystalline direction due to the electrical attraction or repulsion between its attached electronic charge distribution and the neighboring charged ions (Engdahl 1999).

Heisenberg's principal result is contained in the well known simultaneous equations:

$$
\begin{aligned}
\mathrm{y} & =\tanh (\mathrm{x}) \text { for } \mathrm{J}<1 / 2 \\
x & =\left[\beta-\frac{\beta^{2}}{Z}\right]\left(\frac{y}{2}\right)+\left[\frac{y^{3} \beta^{2}}{4 Z}\right]+\frac{\mu_{\beta} \mathrm{B}_{x}}{k T} \\
y=B_{J}(z) & =\left[\frac{2 j+1}{2 j}\right] \operatorname{coth}\left[\frac{(2 j+1) z}{2 j}\right]-\frac{1}{2 j} \operatorname{coth} \frac{z}{2 j} \text { for } \mathrm{J} \geq 1 / 2
\end{aligned}
$$

$\beta=$ is the number of nearest atomic neighbors in the crystal multiplied by the Heisenberg exchange integral (Chen 1986) divided by kT. M is the magnetiza- 
tion in a magnetic field, B (MKS* units), strong enough to orient the large crystal spin and to overcome the small interactions of the electrons. Such interactions are largely responsible for direction effects, remanence, hysteresis, etc., which are unexpected of the unmodified Heisenberg model. $M_{0}$ is the magnetization in total saturation. The $\mu_{\mathrm{B}} \mathrm{B} / \mathrm{kT}$ term is the Bohr magneton multiplied by an applied external magnetic field divided by $\mathrm{kT}$. A consequence of the simultaneous solution of (1) in the limit of $y \rightarrow 0$ and zero external field, is a defining expression for the Curie temperature, $\Theta$, in terms of $\mathrm{J}$ e and $Z$.

Collectively, these equations represent the traditional Heisenberg-Weiss formulation for a spin, $S=1 / 2$, system. For systems with total angular momentum, J > $1 / 2$, the $\tanh (\mathrm{x})$ function of (1) above must be replaced with the more generalized Brillouin function, $\mathrm{B}_{j}(\mathrm{x})$, characteristic of the Weiss formulation. Terfenol-D has $\mathrm{J}>1 / 2$, but analyses of experimental data (Trovillion et al. 1999) indicates a better quality fit when $\mathrm{J}=\mathrm{S}=1 / 2$, and this result is consistent with similar earlier measurements of Curie temperature versus magnetization using $\mathrm{H}-\mathrm{W}$ formulations for the J $>1$ systems of $\mathrm{Fe}, \mathrm{Co}$, and $\mathrm{Ni}$. This departure forms the basis of a beginning in which the external magnetic field, $B_{x^{\prime}}$ of $(1)$ is replaced by an internal field, $B_{i}$, that is stress-generated through magnetostriction within a typical $45 \mu \mathrm{m}$ sized tagging particle. These particles are arranged in linear stringlike "chains," and the collective influence of many such magnetic dipoles upon each other tends to impart a distinct ferromagnetic character to the composite specimen.

The absence of an appreciable external field permits a simplification in that the experimentally measured magnetic field, $\mathrm{B}$, is simply related to the magnetization, so that $\mu_{0} \mathrm{M}=\mathrm{B}$. Thus $\mathrm{y}=\mathrm{M} / \mathrm{M}_{0}=\mathrm{B} / \mathrm{B}_{0}$ in equation (1) and preserves the established $\mathrm{H}-\mathrm{W}$ formalism. An important consequence of this internal magnetic field is to replace the Bohr magneton term, $\mu_{\mathrm{B}}$, in equation (1) with the collective spin magnetic moment, $\mu_{\mathrm{A}^{\prime}}$ characteristic of the C15-type Laves crystal structure of terfenol. The actual number of these spins, $\mathrm{N}$, is defined by the controlling energetics of magnetic domain wall motion. Such motion is a direct consequence of the local value of $\mathrm{B}_{i}$, magnetostrictively generated as a result of the applied material stress acting upon the individual terfenol particles. Thus $\mu_{\mathrm{B}} \mathrm{B} / \mathrm{kT}$ is replaced by $\mathrm{N} \mu_{\mathrm{A}} \mathrm{B}_{\mathrm{i}} / \mathrm{kT}$ in equation (1).

* MKS: meters, kilograms, seconds. 
Following the above format, the magnetic signature of a terfenol-impregnated resin polymer can be cast in the following analytic form:

$$
y=\tanh (\alpha y+\gamma)
$$

Here the $\mathrm{y}^{3}$ term of equation (1) is ignored, and $\alpha, \gamma$, and $\mathrm{B}_{\circ}$ are determined through experiment and quantified by means of the relevant physical parameters associated with the Curie temperature and domain wall physics. $\mathrm{F}$ is the applied force, in kilonewtons, giving rise to the magnetostrictive internal field, $\mathrm{B}_{\mathrm{i}}$. Comparing y coefficients between equations (1) and (2) defines $\alpha$ in terms of $\beta$, and utilizing $\Theta$ as derived from equation (1) permits the elimination of $\mathrm{J}_{e}$ yielding:

$$
\Theta=\frac{T_{o}(1-\sqrt{1-(8 \alpha / Z)})}{1-\sqrt{1-(8 / Z)}}
$$

Equation (3) then defines $\alpha$ in terms of the Curie temperature, $\Theta$, the temperature during experiment (room temperature), $\mathrm{T}_{\mathrm{o}^{\prime}}$ and number of nearest neighbors or coordination number, $\mathrm{Z}$.

Now the actual Curie temperature varies somewhat depending on metallurgical history and alloy stoichiometry. Etrema Products, Inc. (Ames, IA) gives $\Theta=389$ $\pm 20^{\circ} \mathrm{C}$ for their crystalline preparations, but the samples of Trovillion et al. (1999) were prepared from granulated, ball-milled polycrystalline terfenol. Within this range of temperature a corresponding range of $Z$ would be expected for a given $\alpha$. Typical values of $Z$ range from 8 for $\mathrm{Fe}$ to about 12 for $\mathrm{Ni}$ and $\mathrm{Co}$. Actual values of the $\mathrm{C} 15$ cubic lattice constant are reported as $0.73 \mathrm{~nm}$, which is about 10 percent smaller than the $0.81 \mathrm{~nm}$ value derived from this stoichiometry and the inclusion of shared atoms from adjacent unit cells. For terfenol, this tighter packing supports an estimate that a $Z \approx 9$ might be reasonable.

Continuing with equation (3), one can numerically evaluate $\alpha$ for some representative Zs; these computed values appear in Table 1.

Table 1. Room temperature limits on $\alpha$ for various $Z$ and, $\Theta=\mathbf{3 8 9} \pm \mathbf{2 0}{ }^{\circ} \mathrm{C}$.

\begin{tabular}{||ll||}
\hline$Z \approx 8.6$ & $-->0.545<\alpha<0.686$ \\
\hline$Z \approx 8.8$ & $-->0.683<\alpha<0.802$ \\
\hline$Z \approx 9.0$ & $-->0.794<\alpha<0.896$ \\
\hline$Z \approx 9.2$ & $-->0.888<\alpha<0.974$ \\
\hline$Z \approx 9.4$ & $-->0.967<\alpha<1.042$ \\
\hline
\end{tabular}


These results are compared below (see Table 3, page 23) with the values of $\alpha$ deduced from a least squares fit to equation (2), using the data of Trovillion et al. (1999), shown in Figure 2 (see page 24).

The evaluation of $\gamma$ is not so straightforward. However in light of equation (2) and the introductory remarks, it seems reasonable to make the association $\gamma=$ $\mathrm{N} \mu_{\mathrm{A}} \mathrm{B}_{\mathrm{i}} / \mathrm{kT}$ and then quantify the details of just how $\gamma$ depends on the applied force. Unfortunately this requires detailed knowledge of the tagging particle's metallurgical history, dislocation structure, averaged magnetic moment, and the modified elastic and anisotropic constants - none of which is known for the data of Trovillion et al. (1999). The best one can hope for is to make order-ofmagnitude evaluations based on the large-scale physics that is thought to be governing the relevant physical processes.

One begins the description of the various physical parameters found in $\gamma$ with the basic physical argument: the process of magnetostriction converts some of the work of material deformation, resulting from applied stress, into various forms of magnetic energy. The actual details of how this magnetic energy is partitioned within the crystal structure, magnetic domains, and tagging particles can be quite complicated. However the magnetic energy is partitioned, the collective magnetic energy density, $\rho_{\text {Mag }}$ must be of order $\rho_{\text {mench }}$, the mechanical energy density responsible for the magnetostrictive phenomena.

For terfenol-D, this mechanical energy density may be considered to consist of two portions: (1) The traditional Young's modulus-type of energy density and (2) a magnetostrictive equivalent Young's modulus, $Y_{\text {Mag. }}$ Teter, Hathaway, and Clark (1996) demonstrated that for polycrystalline terfenol, $Y_{\text {мag }}$ is the dominant absorber of elastic mechanical energy for applied stress $\leq 15 \mathrm{MPa}$; beyond this stress the traditional $Y_{\text {mech }}$ component is seen to assume asymptotic control. In this asymptotic limit, their data yield a $\mathrm{Y}_{\text {mech }} \approx 50 \mathrm{GPa}$, which is in close agreement with standard values from the literature. Examination of the data from Teter et al. (1996) yields an equivalent $Y_{\text {Mag }}$ function for this system. Prior to onset of "saturation," which is total at $\sim 20 \mathrm{MPa}$, the roughly linear portion of this curve has a slope of $1,100 \mu$ strain at $10 \mathrm{MPa}$, and therefore yields a value of 9.09 GPa for $Y_{\text {Mag. }}$ This value defines the fundamental metric of $\rho_{\text {menh }}$, so for the ge ometry of Trovillion et al. (1999):

$$
\rho_{\text {mech }} \approx 3,760 \mathrm{~Pa}
$$

The above $\rho_{\text {men }}$ defines the magnitude of the equivalent magnetic energy density, from which a measure of $B_{i}$ may be obtained. This $B_{i}$ is envisioned to reside col- 
lectively within the individual tagging particle which is taken to be (roughly) spherical in shape and having a net magnetic moment $\mathrm{M}$, derived from magnetostriction. Equating the magnitude of $\rho_{\text {маg }}$ to that of $\rho_{\text {mech }}$ defines $\mathrm{B}_{i}$ yielding:

$$
B_{i} \approx 0.1375 \text { tesla }
$$

Comparison with the analytic form of equation (2), then implies that the $B_{i}$ portion of $\gamma$ has the magnitude of $B_{i} \approx 0.1375$ tesla.

A field of this order cannot freely exist within a typical $45 \mu \mathrm{m}$ particle but instead is contained within multiple connected magnetic domains. The total magnetic energy of such a system is thereby reduced, and the number of likeoriented spins typically contained within a domain is constrained by the crystal's anisotropy constant, $\mathrm{K}$, and any preferential magnetization axes. It is this characteristic number that defines the $\mathrm{N}$ in the definition of $\gamma$ above. However with increasing magnetic energy density $\rho_{\text {Mag }}$ becomes comparable to $\mathrm{K}$ and the domain walls continue to readjust, minimizing the total crystal energy. Standard treatments (Kittel 1976) of these concepts show that for a (180 degree spin rotation) wall energy (per unit area), $\sigma_{\text {wall, }}$ is related to $\mathrm{K}$ and $\mathrm{N}$ as:

$$
\sigma_{\text {wall }}=2 a N K \text {, for the lattice constant, } a \text {. }
$$

The results of equation (6) provide some guidance as to the sensitivity of the constituent physical parameters. However the value of the anisotropy constant, K, is very sensitive to temperature variations near room temperature, and in fact changes sign [8] near $305^{\circ} \mathrm{K}$; further, it also depends strongly on composition (Koon and Williams 1978) and presumably metallurgical history. With this in mind Clark, Tanner, and Savage (1982) attempted to calculate some typical terfenol-D values for $\sigma_{\text {wall }}$ and the wall thickness $\mathrm{L}=\mathrm{Na}$ using qualitatively similar formulations to equation (6) above. Their results depended somewhat on the relative wall angles found in the terfenol crystal structure and choice of $K$. This term includes both elastic and magnetoelastic energy terms and is listed as about $2.4 \times 10^{4} \mathrm{~J} / \mathrm{m}^{3}$ at $300^{\circ} \mathrm{K}$. These calculations employed standard mean field theory to approximate the exchange constant term, $\mathrm{J} \mathrm{e}^{2}$, from which they obtained typical values for $\sigma_{\text {wall }} \approx 2 \mathrm{ergs} / \mathrm{cm}^{2}$. Combining this result with those of equation (6), it is found that a typical number of spins per domain to be of order $\mathrm{N} \approx 57$.

To complete the calculation of $\gamma$ a determination of the collective spin magnetic moment, $\mu_{\mathrm{A}^{\prime}}$ for the terfenol unit cell must be made. The rare earth (Gd to Yb) atoms have the interesting feature of retaining essentially the same magnetic 
moments and physical shape independent of their local environments as determined by elements, alloys, compounds, and oxides (Engdahl 1999). The magnetic properties and atomic shapes of the transition metals (Sc to Zn), by contrast, very much depend on local crystal structure and chemistry, with the resultant magnetic moment primarily due to spin. Reported values for $\mathrm{Fe}$ vary from $1.6 \mu_{\mathrm{B}}$ to $2.2 \mu_{\mathrm{B}}$ depending upon local environment (Engdahl 1999; Clark, Tanner, and Savage 1982). Thus Fe, Tb, and Dy's contribution to the magnetic moment of terfenol may be taken as a weighted average of their experimental atomic magnetic moment values. For Tb this value equals $9.3 \mu_{\mathrm{B}}$, and for Dy it equals $10.2 \mu_{\mathrm{B}}$, while the value for Fe may be taken of order $1.9 \mu_{\mathrm{B}}$ (Engdahl 1999; Clark, Tanner, and Savage 1982). The net result is:

$$
\mu_{A}=4.67 \mu_{B}
$$

Finally completing the evaluation of $\gamma=\mathrm{N} \mu_{\mathrm{A}} \mathrm{B}_{\mathrm{i}} / \mathrm{kT}$, using the results of equations (5), (6), and (7) at a room temperature of $294^{\circ} \mathrm{K}$ yields a $\gamma \approx 1 /(4.98)$. Given some of the uncertainties that accompany the evaluation of $\gamma$ one can estimate that this numerical value is probably too large or too small by a factor of three or four. Results of the above computation are summarized in Table 2 as follows:

Table 2. Room temperature evaluation of $\gamma=N \mu_{A} B_{i} / k T$.

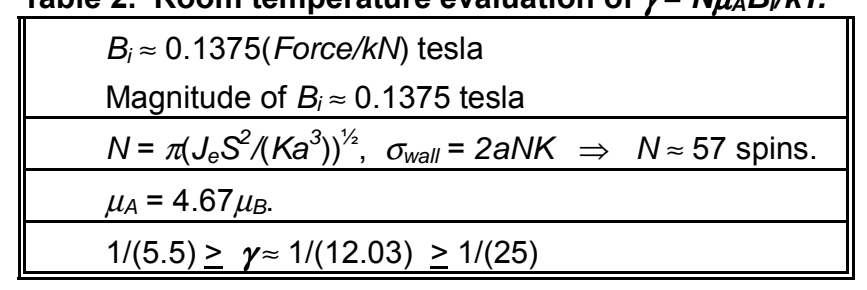

The general analytic form of equation (2) permits a least squares fit to the experimental data of Trovillion et al. (1999). The deduced fitting parameters are presented in Table 3, and are to be compared to the estimated values of $\alpha$, and $\gamma$ appearing in Tables 1 and 2, respectively. Using Table 3, one can numerically evaluate (2) for a series of applied forces; the results are shown in Figure 2, plotted against the normalized experimental data.

Table 3. Least squares determinations of $\alpha, \gamma$, and $B_{o}$ as defined in Equation (3). All parameters have $a \pm 4 \%$ error.

$\alpha=0.82255, \gamma=1 /(12.334), B_{o}=4.4546 \times 10^{-4}$ tesla 


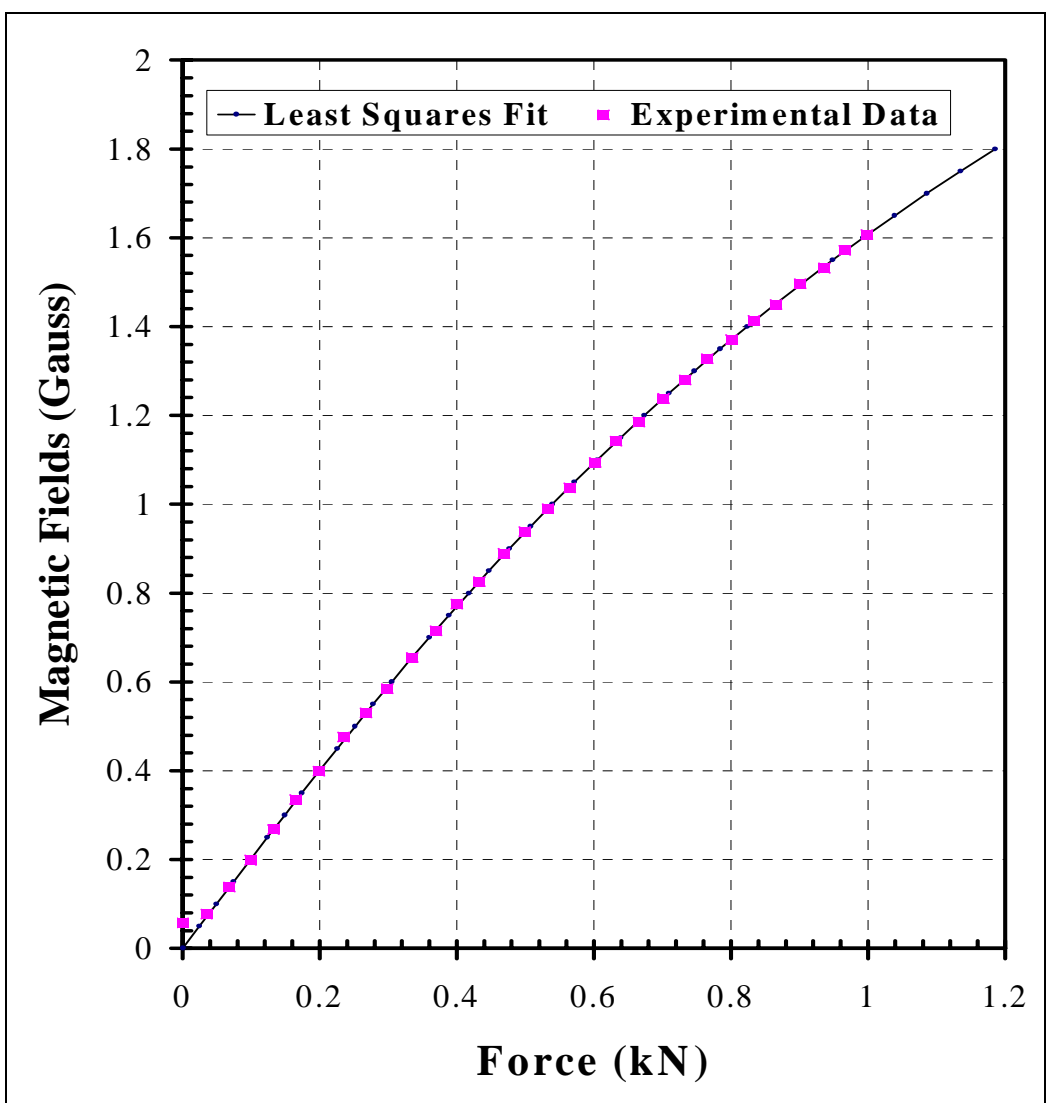

Figure 2. Comparison of the normalized experimental data of Trovillion et al. (1999) to the three-parameter least squares fit of Equation (2) cast in the form of $B=B_{o} \tanh \left(\alpha B / B_{o}+\chi(F / k M)\right)$, and using the values of $\alpha, \gamma$, and $B_{o}$, found in Table 3.

Using the values shown in Table 3, the general fit of equation (2) to these data is seen to be quite good. These normalized data are created by subtracting any constant background component, defined at zero applied force, from the raw experimental data.

This background component originates from a number of sources: stray laboratory fields, residual chemical and material stress occurring during sample preparations (Hommema 1999), and the net residual magnetization following sample polarization but prior to stress loading (Brouwers 1998). These residual stresses can result in a significant component to the background magnetic field so that the actual magnetic response from the loaded sample may not rise above this background until a tensile stress $\approx 0.3 \mathrm{MPa}$ has been achieved. This residual magnetic component (of order $6.3 \mu \mathrm{T}$ ) is clearly shown in Figure 2 as an offset in the data of Trovillion and colleagues (1999) for small initial applied forces. As observed previously, these residual magnetic effects are not expected to be resolved using standard $\mathrm{H}-\mathrm{W}$ theory. Thus these initial (two) points were removed from the data set prior to the fitting procedure. 
One particular consequence of the analytic form of equation (2) is that it predicts a maximum magnetic signature of $\mathrm{B}_{\mathrm{o}}=4.45 \times 10^{-4}$ tesla for very large stresses, which is a testable prediction using the samples of Trovillion et al. (1999). No attempt has been made here to analytically quantify $\mathrm{B}_{\mathrm{o}^{\prime}}$ since it depends upon the details of sample geometry, tagging particle distribution, the abovementioned residual stresses, and metallurgical history. Ongoing research will attempt to quantify some of the residual magnetic field component and its sensitivity to applied stress.

\section{Summary of the Modified H-W Model}

A simple, semi-quantitative phenomenological model has been developed that provides an effective parameterization of the physical parameters that govern the magnetic signatures of a magnetostrictive composite specimen in unidirectional tension. This model can further enhance the utility of magnetostrictive materials employed as tagging agents to augment advanced materials performance. The model makes a testable prediction as to the magnetic field parameter, $\mathrm{B}_{\mathrm{o}^{\prime}}$ found in the data of Trovillion and others (1999). The model further develops estimates, to within a factor of 2 (or better), of the remaining parameters found in equation (2).

\section{Mechanistic Approach: Magnetoelasticity Theory}

Magnetoelasticity theory provides another approach to modify the microscopic behavior of composites. Magnetostrictive materials exhibit a coupled response between their elastic and magnetic behaviors. This coupling is expressed mathematically as a coupling between the elasticity and Maxwell equations through a piezomagnetic tensor, $\mathrm{d}_{\mathrm{ij}}$. When all terms in $\mathrm{d}_{\mathrm{ij}}$ are zero, the equations are decoupled as in a non-magnetostrictive material. The two constitutive equations describing magnetostrictive behavior are

$$
\varepsilon_{i}=S_{i j}^{H} \sigma_{j}+d_{i j} H_{j}
$$

and

$$
B_{i}=d_{i j} \sigma_{j}+\mu_{i j}^{\sigma} H_{j}
$$

where $\varepsilon_{i}$ is strain, $\sigma_{\mathrm{j}}$ is stress, $\mathrm{H}_{\mathrm{j}}$ is magnetic field, $\mathrm{S}_{\mathrm{ij}}{ }_{\mathrm{ij}}$ is the compliance tensor at constant field $\mu_{i j}^{\sigma}$ is the magnetic permeability tensor at constant stress, and $B_{i}$ is the magnetic flux vector. 
In the current analysis, special emphasis is placed on equation (9). To account for the nonlinear behavior in the magnetic field equation as magnetic saturation is approached, a nonlinear formulation analogous to electrostrictives is adopted (Damjanovic and Newnham 1992), expressed as follows:

$$
B_{i}=d_{i j} \sigma_{j}+\frac{1}{2} M_{i j k} \sigma_{j} \sigma_{k}+\mu_{i j}^{\sigma} H_{j}
$$

where $\mathrm{M}_{\mathrm{ijk}}$ is the magnetostrictive tensor.

Equation 10 is simplified here because the current experiments focus on uniaxial loading in the z-axis. In addition, because the tests are taken at a single point in space, extraneous $H_{j}$ effects (e.g., Earth's magnetic field) can be removed from the relationship. Consequently, equation (10) can be reduced to:

$$
B=B_{3}=d_{33} \sigma+\frac{1}{2} M_{333} \sigma^{2}
$$

where $d_{33}$ and $M_{333}$ are the piezomagnetic and magnetostrictive coefficients, respectively, for the magnetostrictive composite. 


\section{Specimen Fabrication and Testing Program}

\section{Materials and Fabrication Methods}

Amorphous ingots of terfenol-D purchased from Etrema Products, Inc. (Ames, IA) were ground into a powder and classified into different size ranges. Grinding was carried out under a nitrogen atmosphere to prevent oxidation. In this study particles $<45 \mu \mathrm{m}$ in size were used. The specimens tested in this study were made from terfenol-D particles embedded in one of two different resin matrices. Some specimens were reinforced with $10 \mathrm{oz} / \mathrm{sq}$ yd plain weave glass woven fabric (\#245 supplied by Fibre Glast, Brookville, OH). Other specimens were resin-only (neat) samples. Specimens were manufactured both at the University of Illinois at Urbana-Champaign (UIUC) and CERL using similar processes and materials (except where noted).

\section{UIUC Specimens}

For the UIUC specimens, a polyester resin (Polylite 31610-05,supplied by Reichhold Chemicals, Research Triangle Park, NC) was first mixed together with the correct amount of two catalysts, dimethylaniline (DMA) and cobalt naphthenate, to provide the desired gel time. A 10-minute gel time was used for the neat resin specimens while a 30-minute gel time was used in the glass-reinforced specimens to allow time for fiber placement. For a 10-minute gel time, 0.1\% DMA and $0.1 \%$ cobalt naphthenate by weight of resin were needed. For a 30-minute gel time, $0.06 \%$ DMA and $0.06 \%$ cobalt naphthenate were used. Then the desired volume fraction of terfenol-D powder was mixed with the resin. Finally, methyl ethyl ketone peroxide (MEKP) was added to initiate the polymerization reaction. For the neat resin (unreinforced) specimens, 1.0\% MEKP by weight of resin was used while $0.75 \%$ was used for glass-fiber-reinforced specimens. To reduce the amount of voids in the specimens an aerator, BYK-A 501, was also added to the polyester resin at a concentration of $0.3 \%$ resin weight.

The resin mixture was degassed in a vacuum oven after each ingredient was added. Once all ingredients were thoroughly mixed and degassed, the mixture 
was poured into the cavities of a silicone rubber mold. The dimensions of all UIUC specimens tested in the current program were $0.25 \times 0.25 \times 7 \mathrm{in}$.

During cure, all of the neat resin specimens and most of the fiber-reinforced samples were placed between two permanent magnets in a process called chaining. In the chaining process the samples are exposed to a strong magnetic field in order to align the terfenol-D particles along an axis perpendicular to the specimen surface. In terms of material design, the purpose of chaining is to maximize the specimen's magnetostrictive response when it is loaded. In the case of the neat resin specimens, however, all samples were chained for a separate practical reason: without exposure to the magnetic field the terfenol-D particles would settle to the bottom of the mold before the resin hardened; the chaining process kept the magnetostrictive particles suspended (i.e., more evenly distributed) through the resin until the specimen was fully cured.

In all cases, whether chained or unchained, the specimens were allowed to cure at room temperature for 24 hours. The mold, the specimens, and the permanent magnets used in the chaining process are diagrammed in Figure 3.

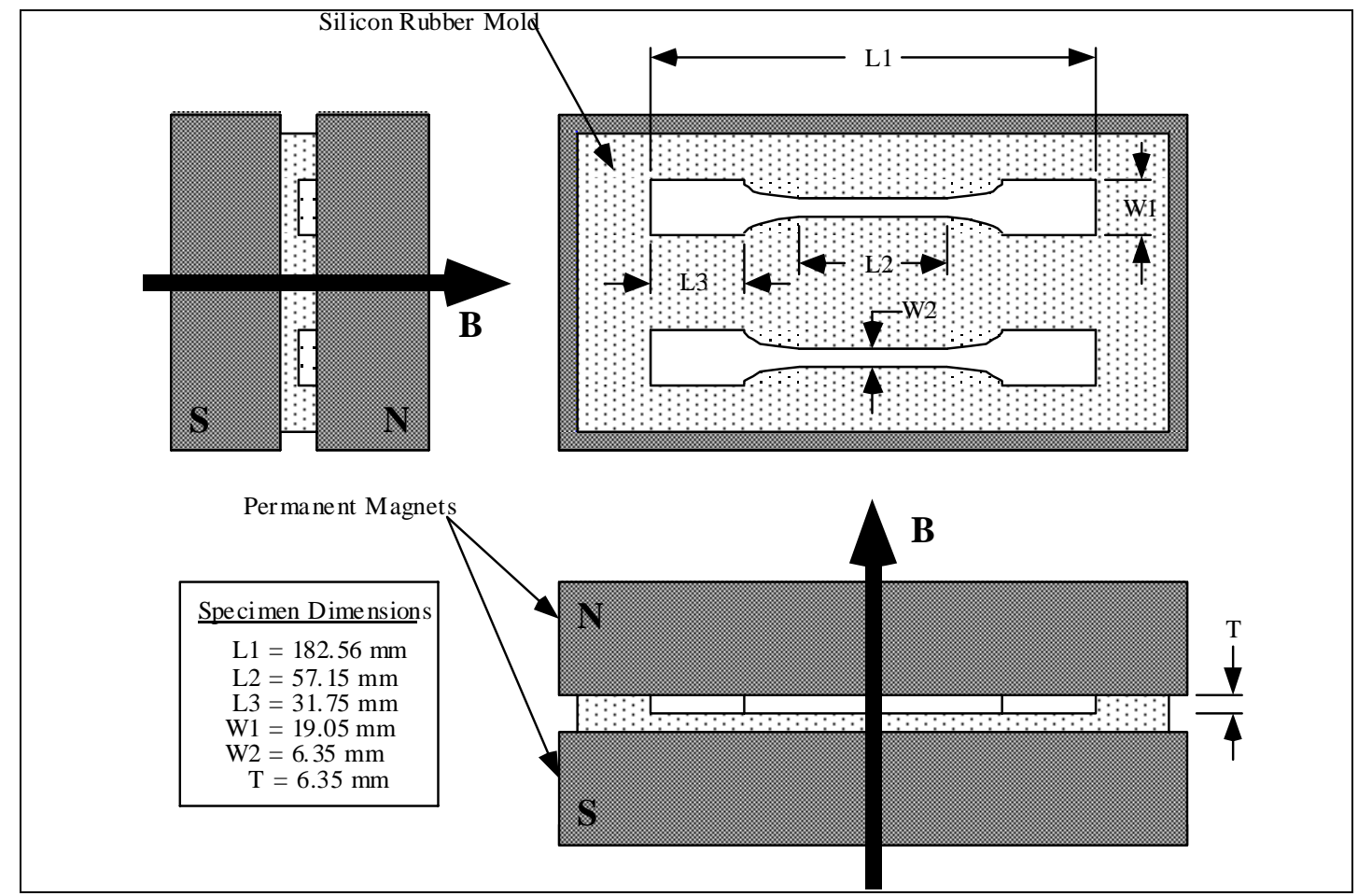

Figure 3. Silicone rubber mold and permanent magnets used during specimen manufacturing. 


\section{CERL Specimens}

The specimens manufactured at CERL were fabricated in a similar fashion to the method used at UIUC. One notable difference was the material selected as the resin matrix: the CERL specimens were made with the epoxy system Epon 828 (Shell) instead of a polyester system. The E pon 828 was mixed in a 1:1 ratio with a curing agent (Epicure 3140) and allowed to cure for 24 hours before re moval from the mold. Another difference was the specimen dimensions: the CERL specimens were molded to a constant width of 0.75 in. as opposed to the narrower 0.25 in. width of the UIUC specimens (see W2 in Figure 3). In other words, the CERL specimens measured $0.75 \times 0.25 \times 7 \mathrm{in}$.

\section{Testing Procedure}

A common coordinate system was implemented for the testing of the magnetostrictive specimens. Figure 4 illustrates this coordinate system. The axial direction is the direction of loading and the transverse direction is perpendicular to the face of the specimen. In the case of specimens that were magnetically chained, the transverse direction is also parallel to the direction of chaining.

All testing, except for the neat resin axial response, was conducted using CERL'S 50 kip MTS (Eden Prairie, MN) servo-hydraulic load frame. Specially designed nonmagnetic grips were employed. Load and actuator displacement data were recorded from the MTS control system. Magnetic field measurements were re corded using a Lakeshore gaussmeter (Westerville, $\mathrm{OH}$ ).

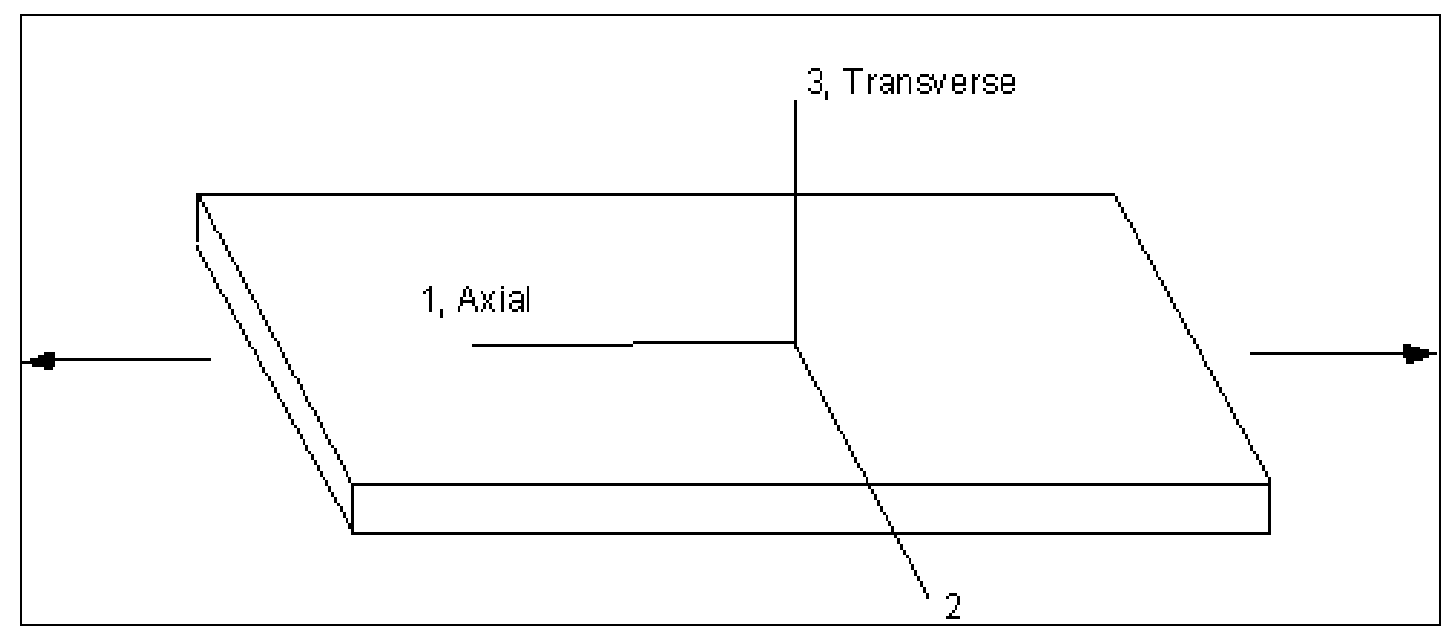

Figure 4. Description of coordinate system used for experiments. 
A process of magnetic annealing was developed and applied during the various testing sequences. In this study the term annealing refers to the application of a magnetic field to align the magnetic dipoles in a magnetostrictive specimen (as opposed to the chaining procedure, which aligns the actual terfenol-D particles in the uncured resin). A specimen is annealed in order to reverse the unrecoverable part of the stress vs magnetic field loop created by domain wall motion - in other words, to eliminate a sample's magnetic history after a specified stress cycle. The parameters and effects of annealing have implications for signal strength in a magnetostrictive member subjected to dynamic loading over time.

Three test protocols were used in this study. Under all protocols the magnetic field measurements were taken in the transverse* direction. The three protocols are described as follows:

- Control Protocol (Zorro Curves). A set of zorro† curves was generated for each specimen tested. This was a simple load control test in which the specimen was placed in the load frame, annealed, then ramped up to a set load and ramped down to zero at the same rate.

- Reset Protocol 1. This test protocol was designed to look at the effect of annealing under progressively increasing stress. Each specimen was annealed, loaded to step 1, annealed, loaded to step 2, annealed, loaded to step 3, annealed, loaded to step 4, and so on until all load steps were applied. The same sequence was applied during progressive unloading.

- Reset Protocol 2. This protocol was designed to look at the effect of annealing under alternate loading and unloading. Each applied load was followed by annealing, complete unloading, annealing, and then the next specified loading step. Specifically, each sample was annealed, then loaded to step 1, annealed again, unloaded to zero, annealed again, then loaded to step 2, annealed once more, unloaded to zero, annealed again, then loaded to step 3, annealed, unloaded to zero, annealed, and so on through the entire loading and unloading sequence.

\footnotetext{
* In initial testing, magnetic field measurements were taken in both the axial and transverse directions. The results of these tests demonstrated that the strongest signals were obtainable from the transverse measurement, so all further measurements made under the three test protocols were taken in the transverse direction.

$\dagger$ This descriptor was chosen because the data plots produced by the control protocol typically resemble the signature mark of the swashbuckling fictional swordsman of the same name, created by Johnston McCulley.
} 


\section{Tension Testing of Tagged Neat Resin Specimens}

The magnetic field produced by the stressing of magnetostrictive particles is a vector quantity. During this research the vector components were measured to identify which component changed the most with respect to the stress. The axial (along the specimen axis) and transverse (perpendicular to the specimen surface) results are presented here.

\section{Axial Response}

After manufacture of the neat resin samples at the University of Illinois, the particle domains were randomly oriented in the composite if no external field was applied. A magnetic field of a particular strength and duration is required to align the magnetic domains of the particles to maximize magnetostriction sensitivity. The annealing procedure employs an Ogallala Model P/N 41C1375 electromagnet (Arnold Engineering, Tullahoma, TN) powered by a Sorenson Model DCS 150-7 programmable direct current (DC) power source and placed $38 \mathrm{~mm}$ from the samples in an orientation perpendicular to the applied load.

Figure 5 shows several curves of axial magnetic flux versus stress for a specimen that was both chained during cure and annealed prior to testing. The same specimen was elastically loaded and unloaded 10 times. Between each run the samples were magnetically annealed perpendicular to the loading direction through the thickness at 2500 gauss for 5 minutes. Good repeatability in the data is observed and an average flux of $0.153 \pm 0.008$ gauss is observed at 12.6 $\mathrm{MPa}$. 


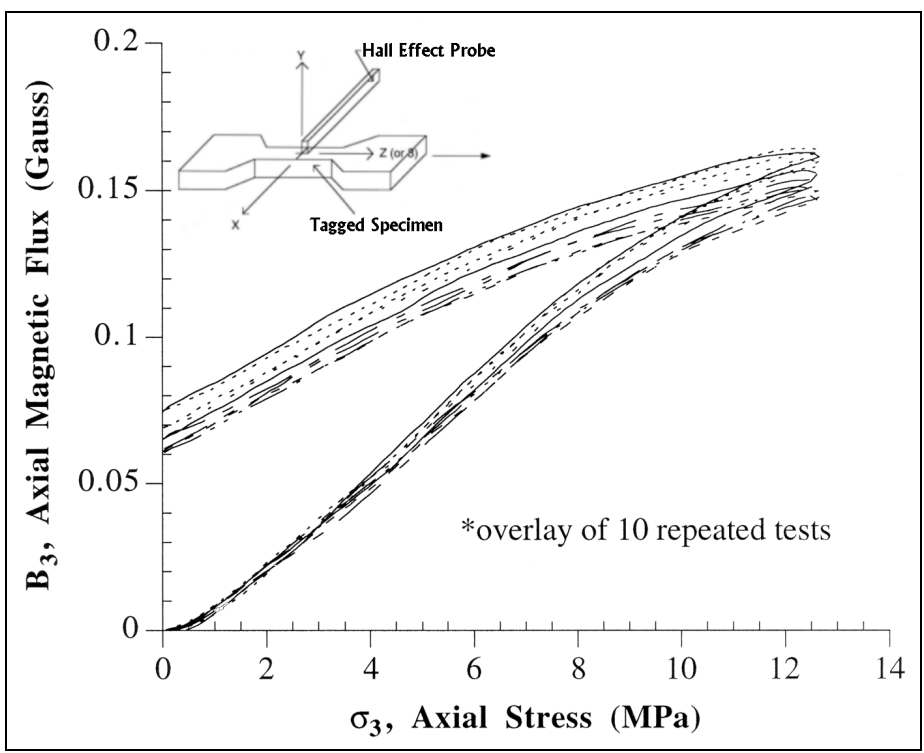

Figure 5. Repeatability tests for a magnetostrictive composite.

The next series of tests (Figure 6) focused on cyclic effects without annealing between the cycles. It can be seen that there is a reduction in total signal change (signal at peak stress minus signal at no load). The signal begins to reach its steady-state response after about 32 cycles with a total change in magnetic flux of 0.050 gauss. The 31 percent signal reduction is due to irreversible domain wall motion. Reannealing of the sample eliminates this magnetic history, however, and returns the signal to the cycle 1 response.

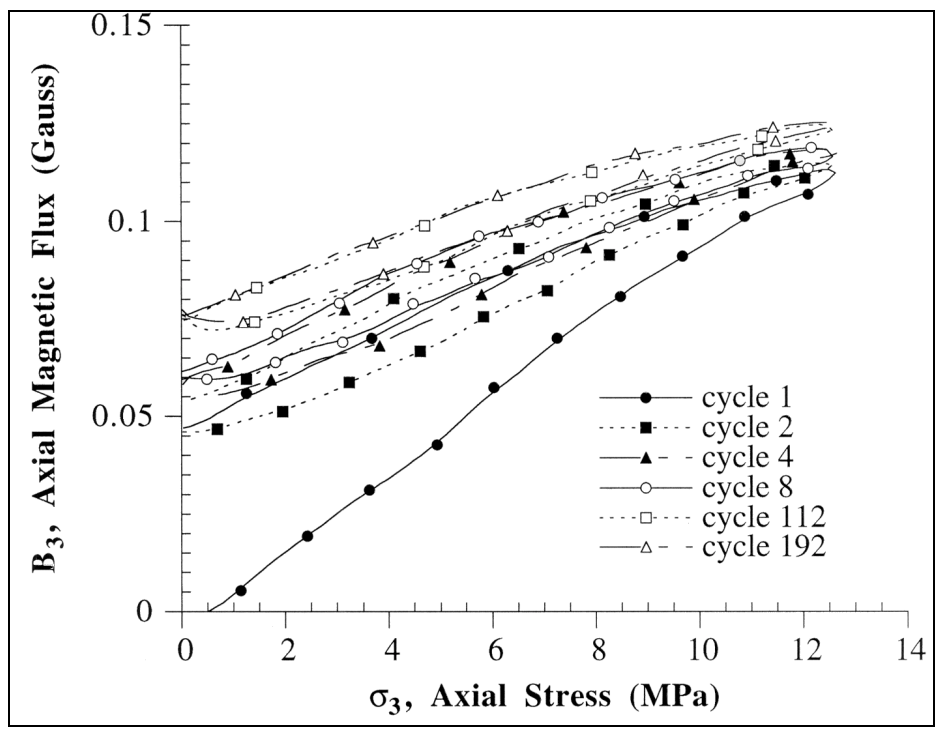

Figure 6. Cyclic tests for a magnetostrictive composite.

The effect of magnetic annealing strength is shown in Figure 7. Using a magnetic field above 400 gauss is sufficient for achieving maximum sensitivity during the test. This maximum sensitivity allows for the use of less sensitive 
gaussmeter equipment for in-field load detection. In various tests conducted during this research it was found that the application of a magnetic field of this strength for times as short as 1 second is sufficient for magnetic annealing.

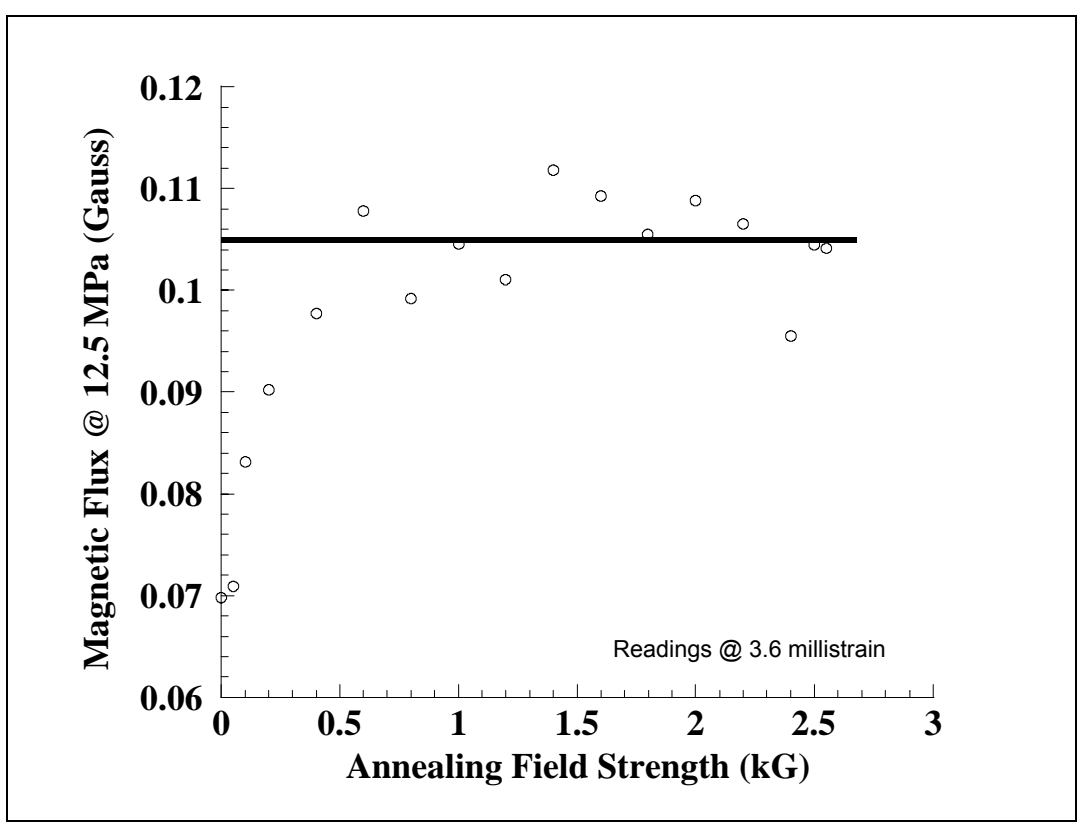

Figure 7. Effect of magnetic annealing field strength on sensitivity.

While annealing is important to magnetic domain alignment it is equally important to study the effects of a magnetic field during cure. Terfenol-D is a highly dense material - almost nine times more dense than the matrix resin - and it settles easily before the polymer matrix material can gel. A low-strength magnetic field applied through the thickness can aid in the suspension of terfenol-D particles in the medium. In this manner, the terfenol-D particles form chains in the direction of the applied field. Specimens manufactured in this fashion are more sensitive compared to a specimen with randomly distributed unchained particles. Figure 8 shows the effect of a magnetic field application during cure (loading curves only). The chained specimens that had been annealed in the thickness direction had a signal 18 times stronger than that of an equivalent unchained specimen (both loaded to $12.6 \mathrm{MPa}$ ).

A curve-fitting process was applied to the loading-only axial flux vs stress experimental data. The piezomagnetic parameters for the magnetostrictive composite, $\mathrm{d}_{33}$ and $\mathrm{M}_{333}$, were extracted for a range of volume fractions. These data are important since an increase in $\mathrm{d}_{33}$ (and $\mathrm{M}_{333}$ ) for the magnetostrictive composite will result in a greater magnetic flux signature. Therefore, maximizing $d_{33}$ is desired for the optimization of load-detection equipment requirements. It can be seen from Figure 9 that for volume fractions below $5 \%$, the piezomagnetic constants change linearly with volume fraction. Similar trends are observed in $\mathrm{M}_{333^{*}}$. 


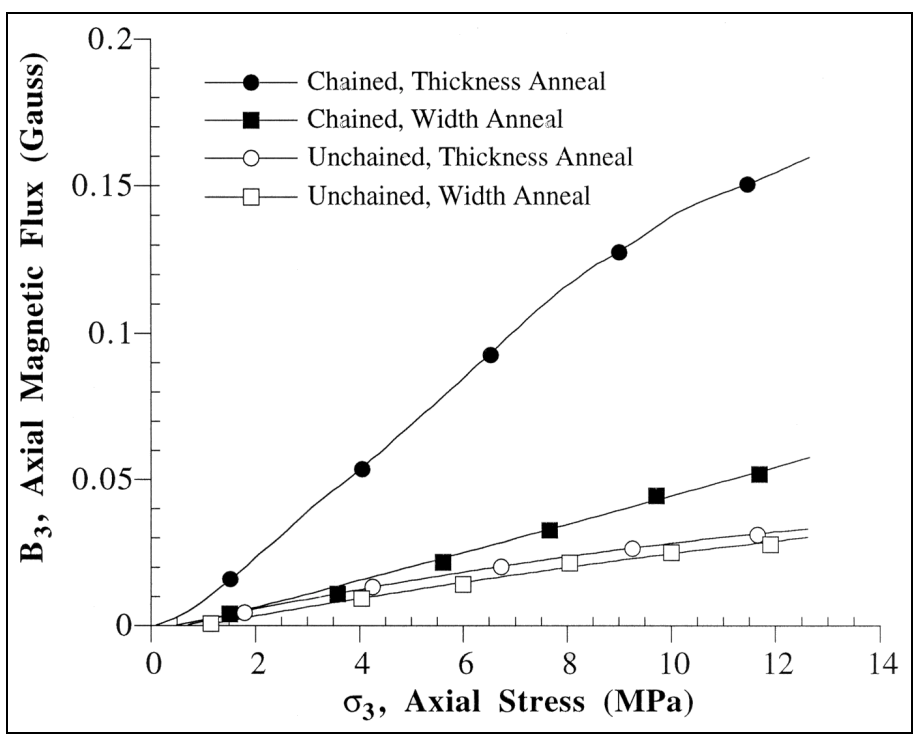

Figure 8. The effect of magnetic field during composite cure.

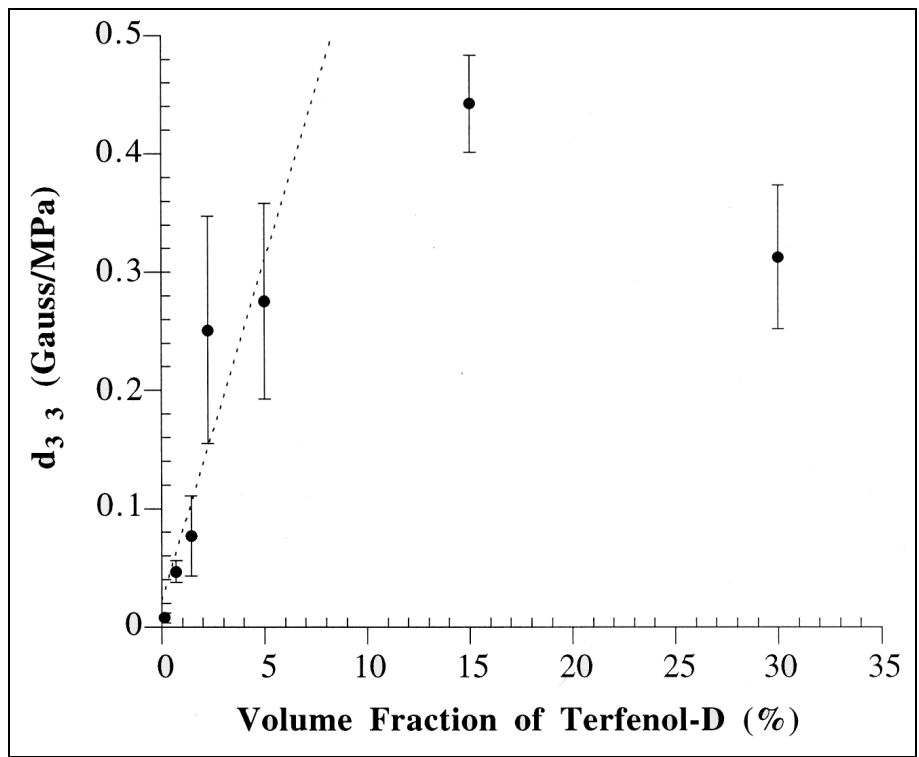

Figure 9. Magnetostrictive composite piezomagnetic coefficient $d_{33}$ vs volume fraction.

The effect of damage on signal response was evaluated. An undamaged sample was strained to 0.0036 and axial field readings were taken at various widths al ong the magnetostrictive composite. A small hole (approximately $3.8 \mathrm{~mm}$ ) was drilled into the specimen and the same axial readings were taken at the same strain. The difference of the two (undamaged and damaged) is plotted in Figure 10. A significant increase in axial field strength is detected close to the damaged region. This is due to the local stress concentration created by the hole and the increased magnetic response observed locally in the terfenol-D particles. 


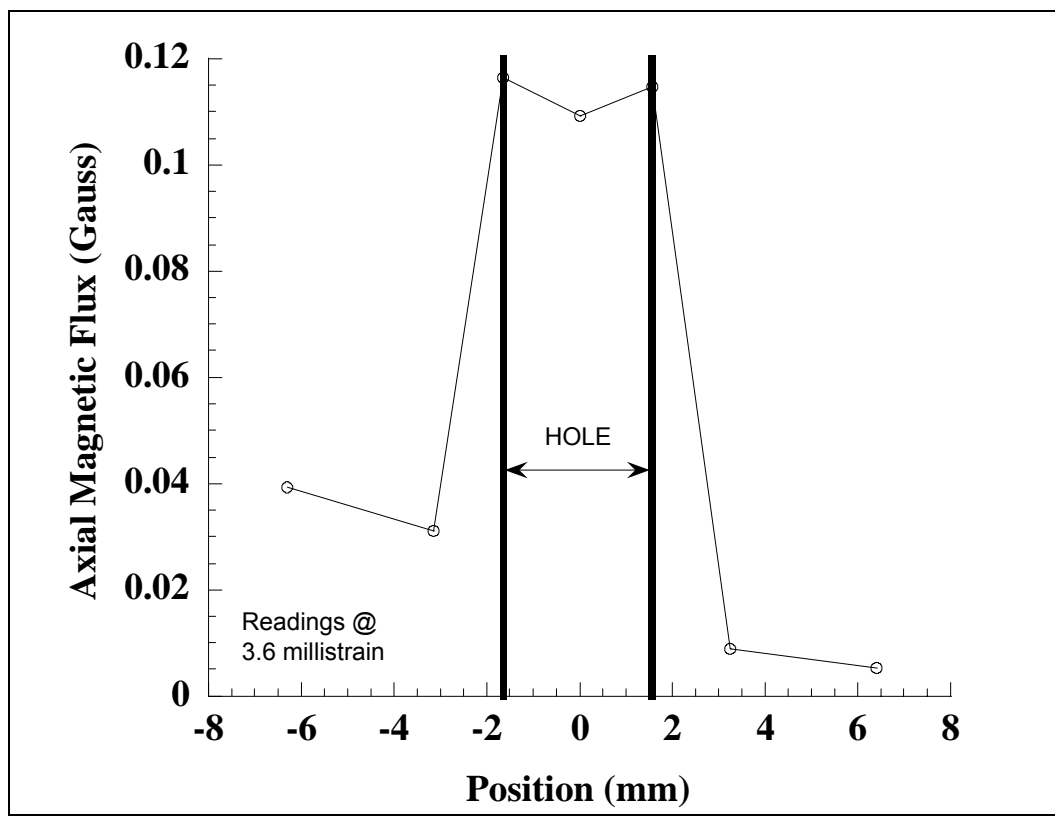

Figure 10. Axial magnetic field vs position in a damage sample.

\section{Transverse Response}

The transverse flux readings turned out to be much stronger than the axial fields, as shown in Figure 11, with a maximum change in the magnetic field of about 0.5 gauss at $1000 \mathrm{psi}$. It is unclear why the transverse flux readings were so much stronger than the axial signals, and this question will be the focus of future research. For current purposes the discussion will be limited to the transverse magnetic fields due to this large difference in response.

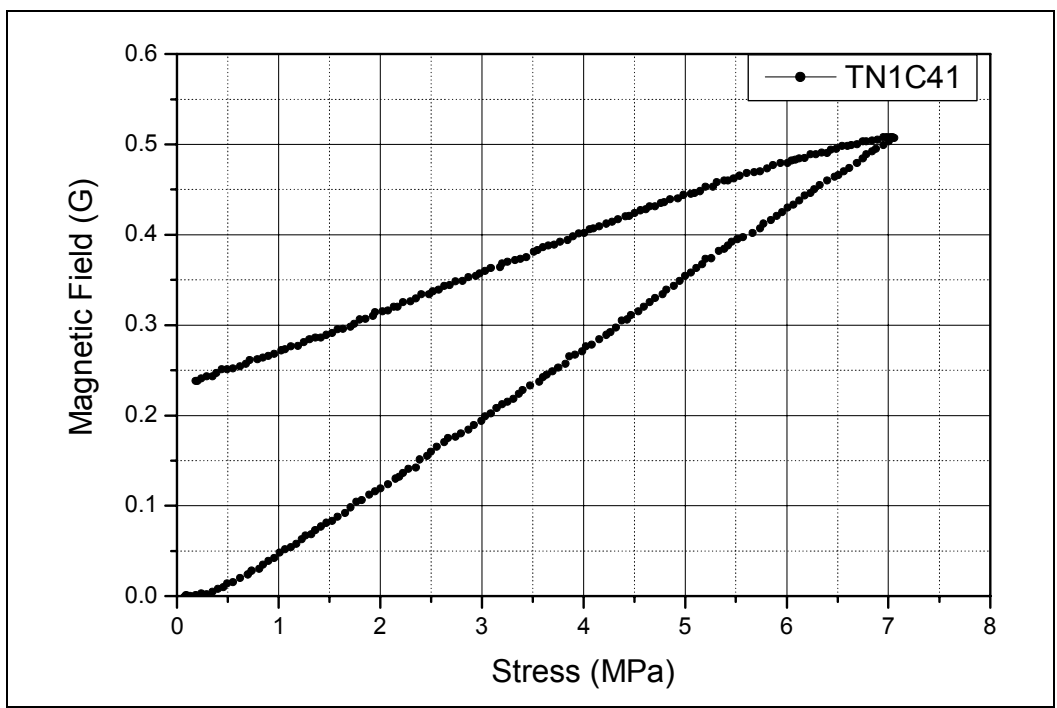

Figure 11. Transverse (x-axis) magnetic flux density vs load, neat resin samples. 


\section{Reset Response of Neat Resin Samples}

The tagged neat epoxy resin samples were tested according to Reset Protocols 1 and 2. The specimens were loaded to maximum load of $250 \mathrm{lb}$ in $50 \mathrm{lb}$ incre ments at $2.5 \mathrm{lb} / \mathrm{s}$ for Reset Protocol 1. They were then loaded to $500 \mathrm{lb}$ in $100 \mathrm{lb}$ increments at $2.5 \mathrm{lb} / \mathrm{s}$ for Reset Protocol 2. Figures 12 and 13 show the forms of typical curves plotted for Reset Protocols 1 and 2, respectively.

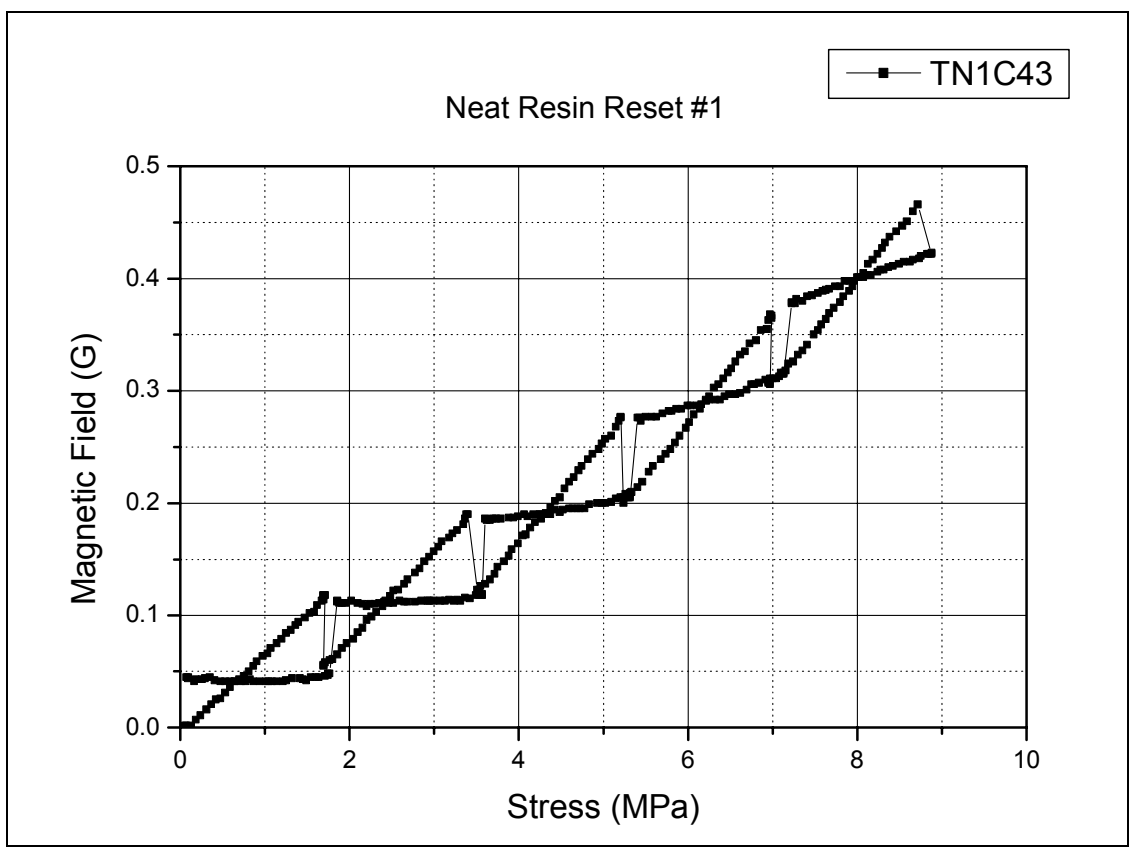

Figure 12. Plot of Reset Protocol 1, neat resin specimen.

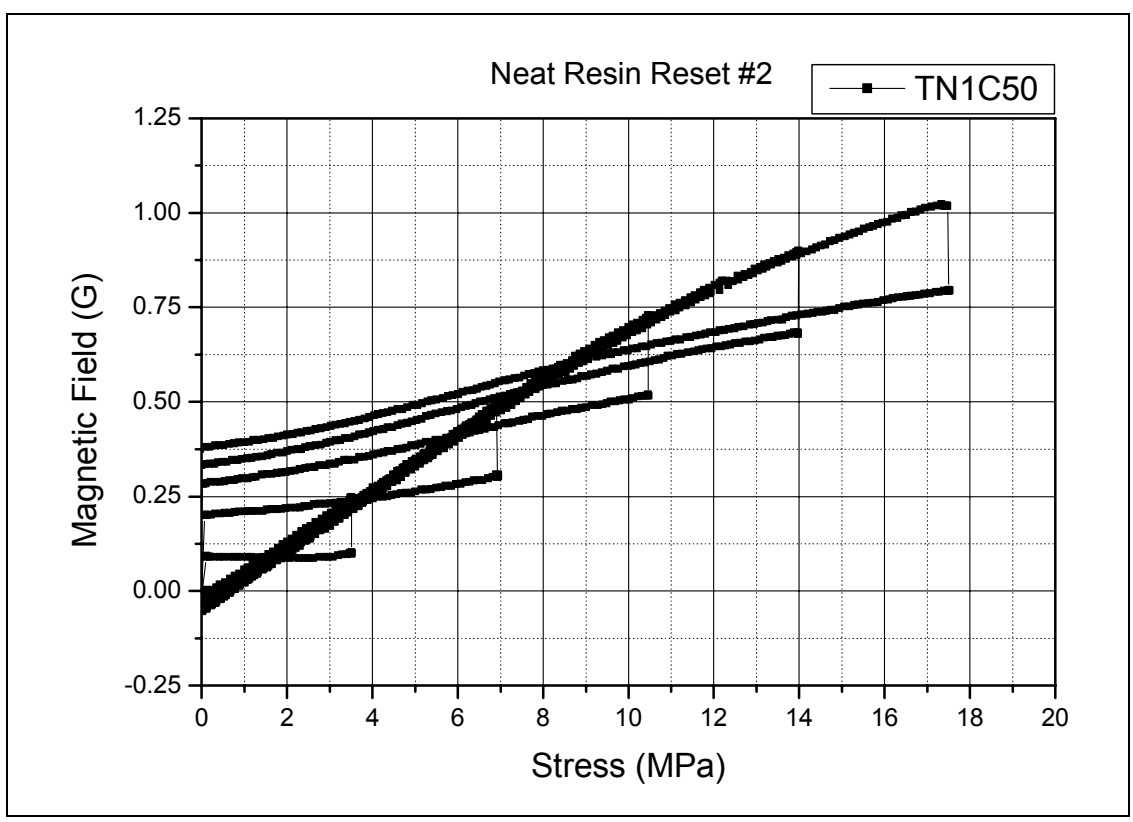

Figure 13. Plot of Reset Protocol 2, neat resin specimen. 
A summary of Reset Protocols 1 and 2 is given in Figures 14 and 15, respectively. These figures show the relationship between the magnetic field (before and after anneal) and the stress at which the specimen was annealed.

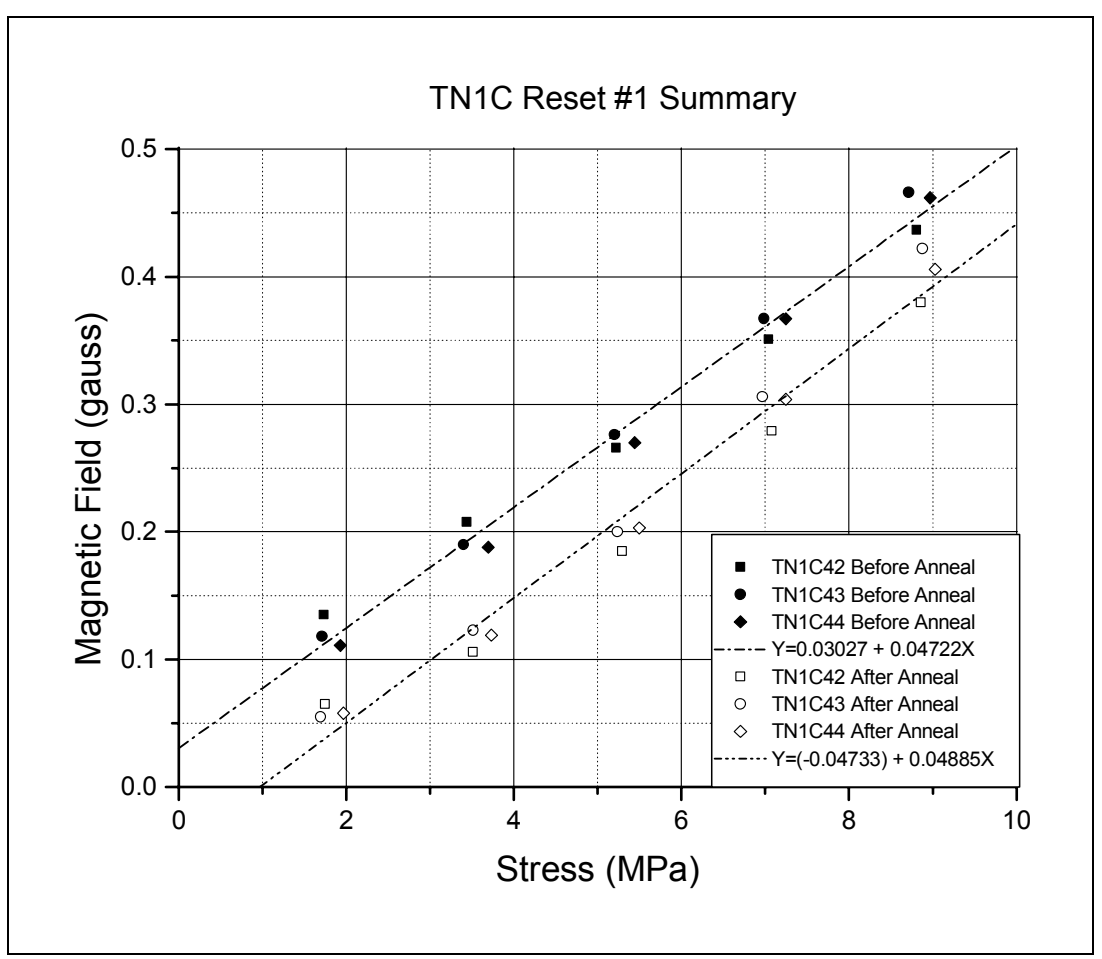

Figure 14. Summary plot for Reset Protocol 1, neat resin specimen.

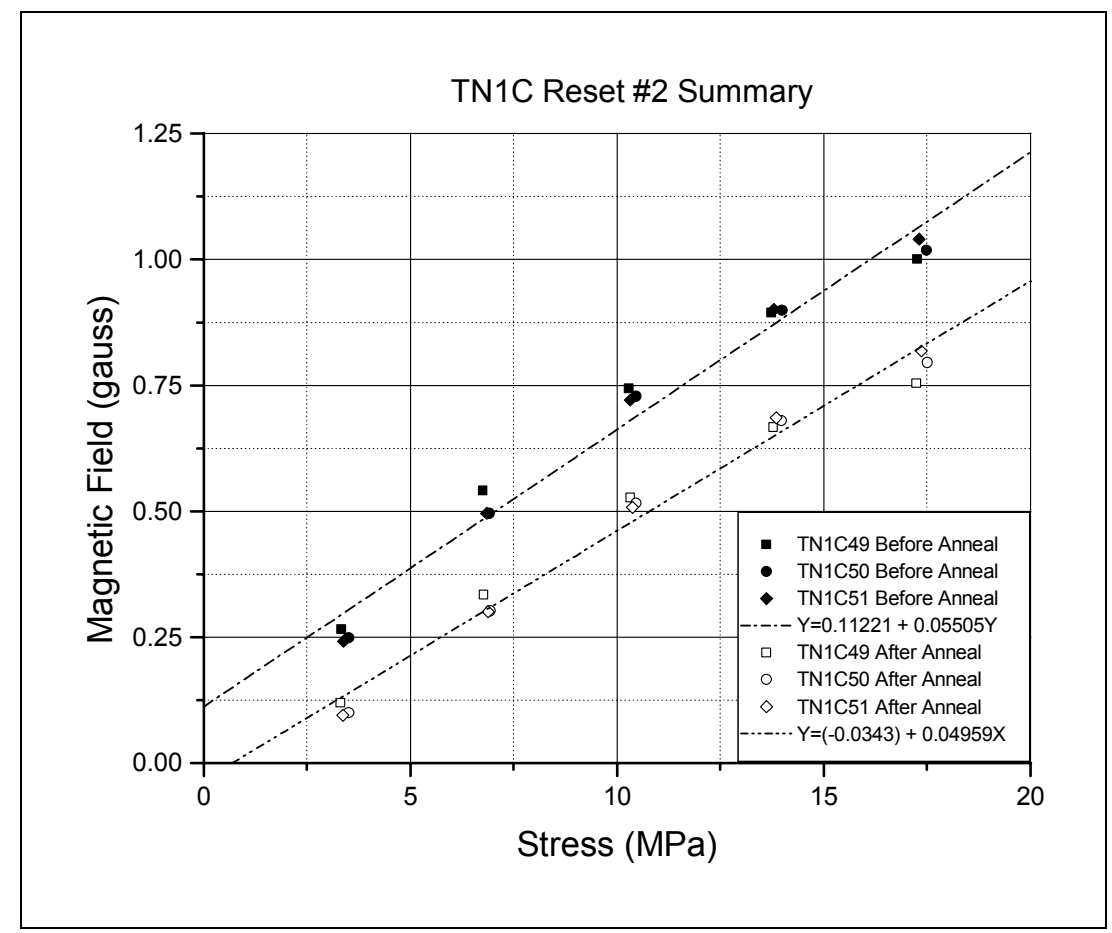

Figure 15. Summary plot for Reset Protocol 2, neat resin specimen. 


\section{Tagged Composite Samples Loaded in Tension}

The tagged fiber-reinforced composite samples were manufactured at CERL with unidirectional glass fibers according to the procedure described on page 29. Both chained and unchained specimens were manufactured. These samples were tested using the three protocols outlined in Chapter 3. Only the transverse response was tested as a result of the findings for the neat resin sample tests reported in Chapter 4.

\section{Transverse Response}

The transverse response of the fiber-reinforced samples under the Control Protocol was similar to that of the neat resin samples. The difference lies mainly in the magnitudes of the magnetic field and the amount of hysterisis observed. Figure 16 shows the transverse response for a chained and anneal ed specimen, and Figure 17 shows the transverse response for an unchained and annealed specimen.

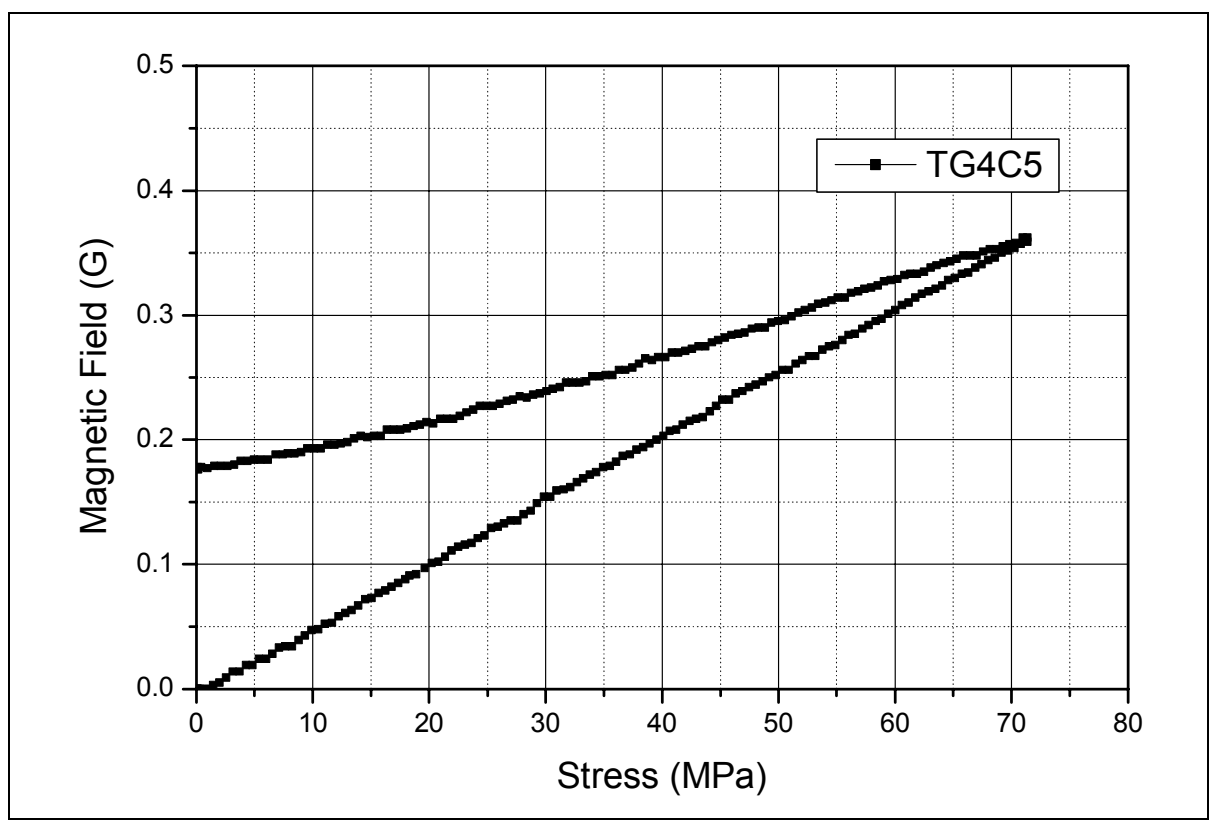

Figure 16. Typical zorro curve for chained composite specimen with annealing. 


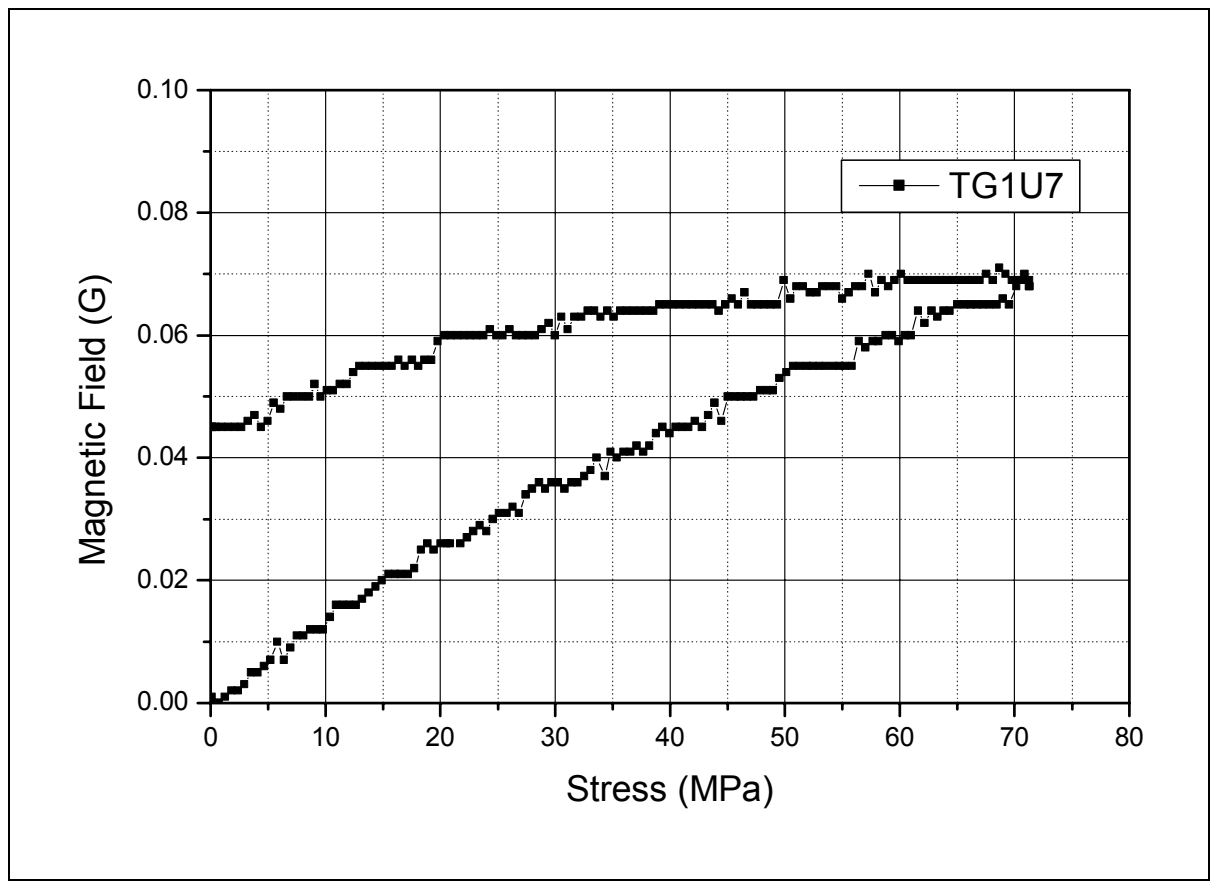

Figure 17. Typical zorro curve for unchained composite specimen with annealing.

\section{Reset Response of the Composite Samples}

The specimens were then tested using Reset Protocols 1 and 2. The specimens were stressed to maximum load of $2000 \mathrm{lb}$ in $500 \mathrm{lb}$ increments at $25 \mathrm{lb} / \mathrm{s}$ for Reset Protocol 1. Subsequently, they were loaded to $2000 \mathrm{lb}$ in $500 \mathrm{lb}$ increments at $25 \mathrm{lb} / \mathrm{s}$ for Reset Protocol 2. Figures 18 and 19 are typical curves for Reset Protocols 1 and 2, respectively, for the specimen with a curing field applied. Figures 20 and 21 are typical curves for Reset Protocols 1 and 2, respectively, for the specimen without a curing field applied.

A summary of data for Reset Protocols 1 and 2 is given for the chained specimens in Figures 22 and 23, respectively. A summary of data for Reset Protocols 1 and 2 for the unchained specimen is given in Figures 24 and 25 . These figures show the relationship between the magnetic field before and after anneal to the stress at which the specimen was annealed. 


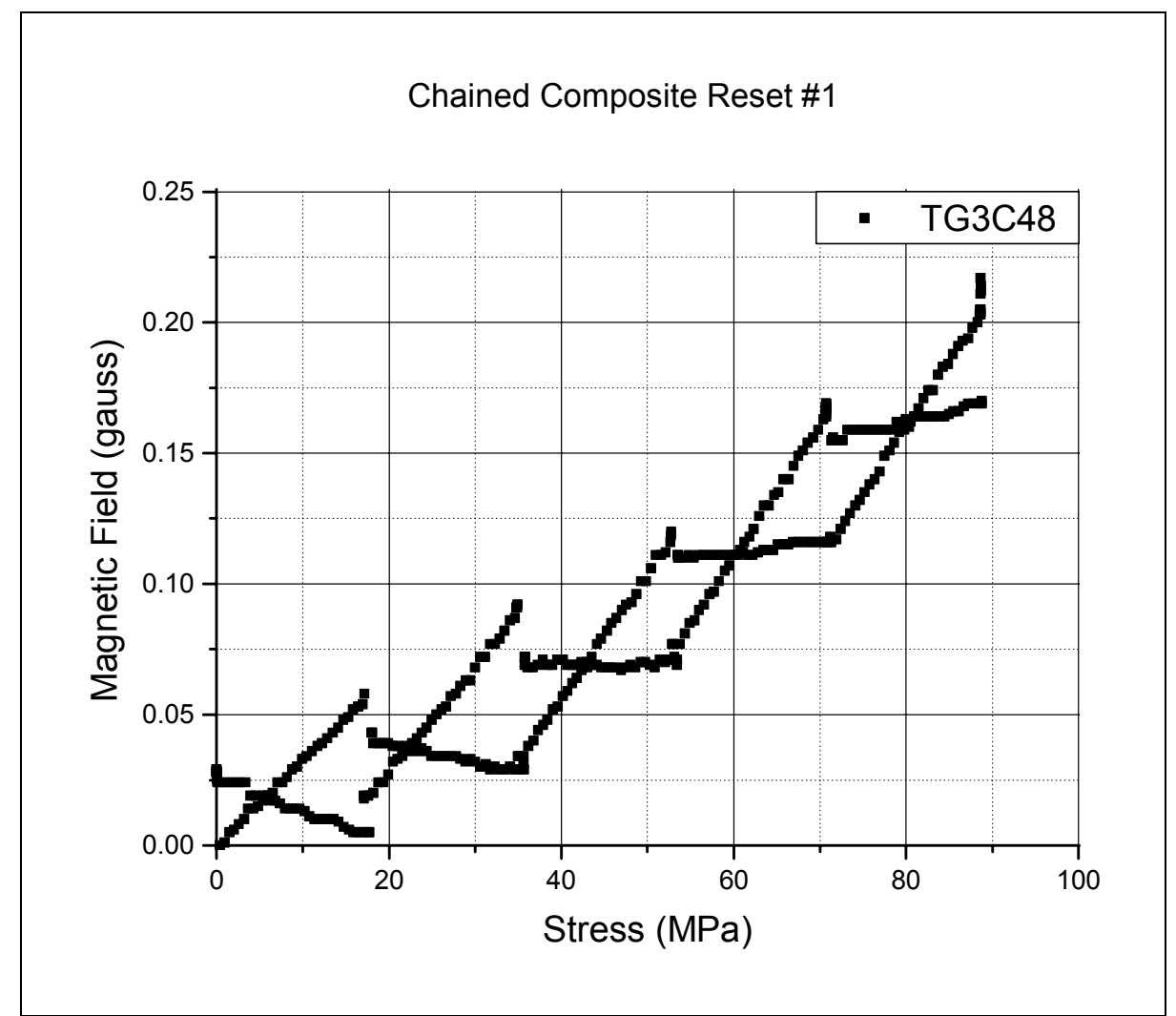

Figure 18. Plot of Reset Protocol 1, chained composite specimen.

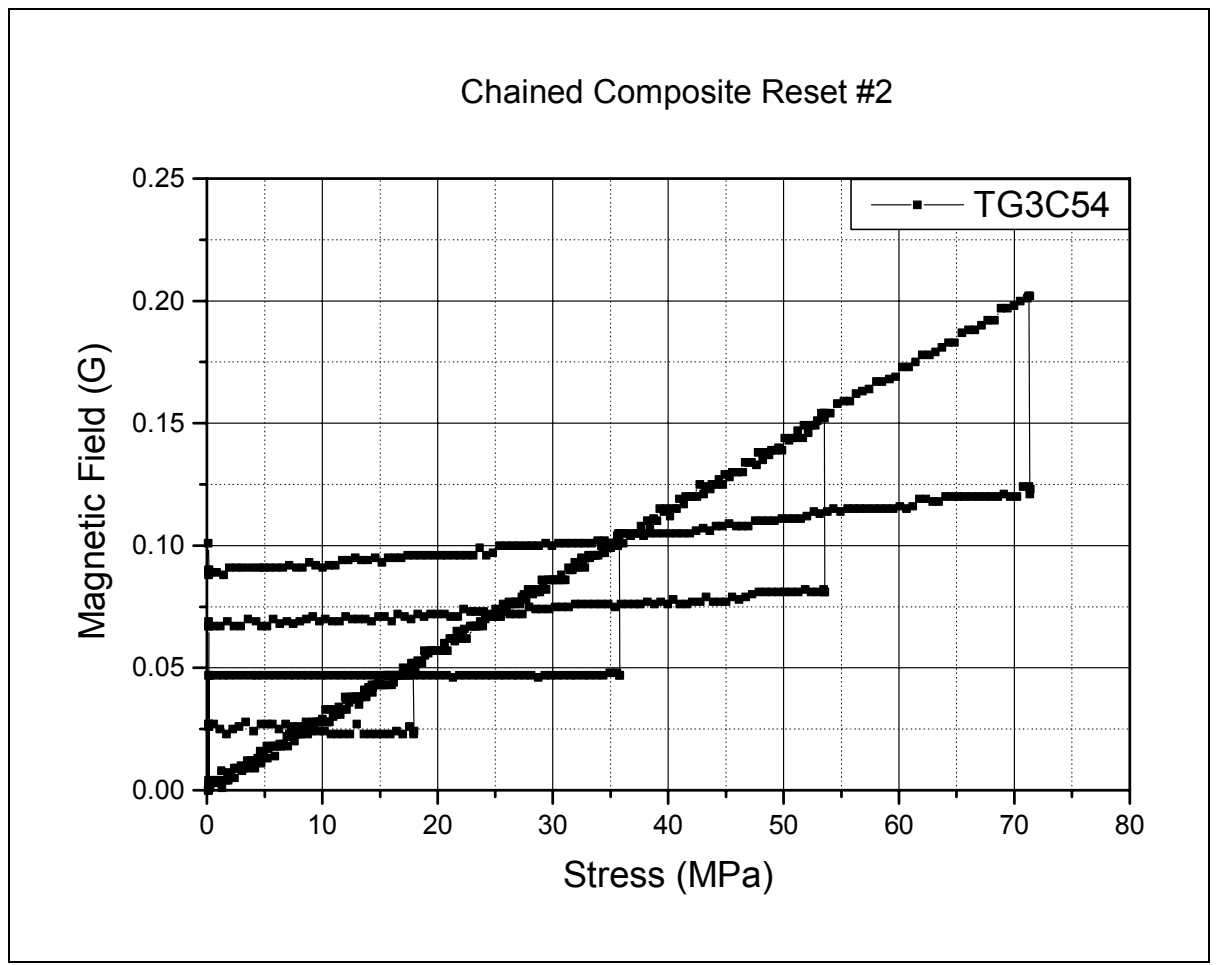

Figure 19. Plot of Reset Protocol 2, chained composite specimen. 


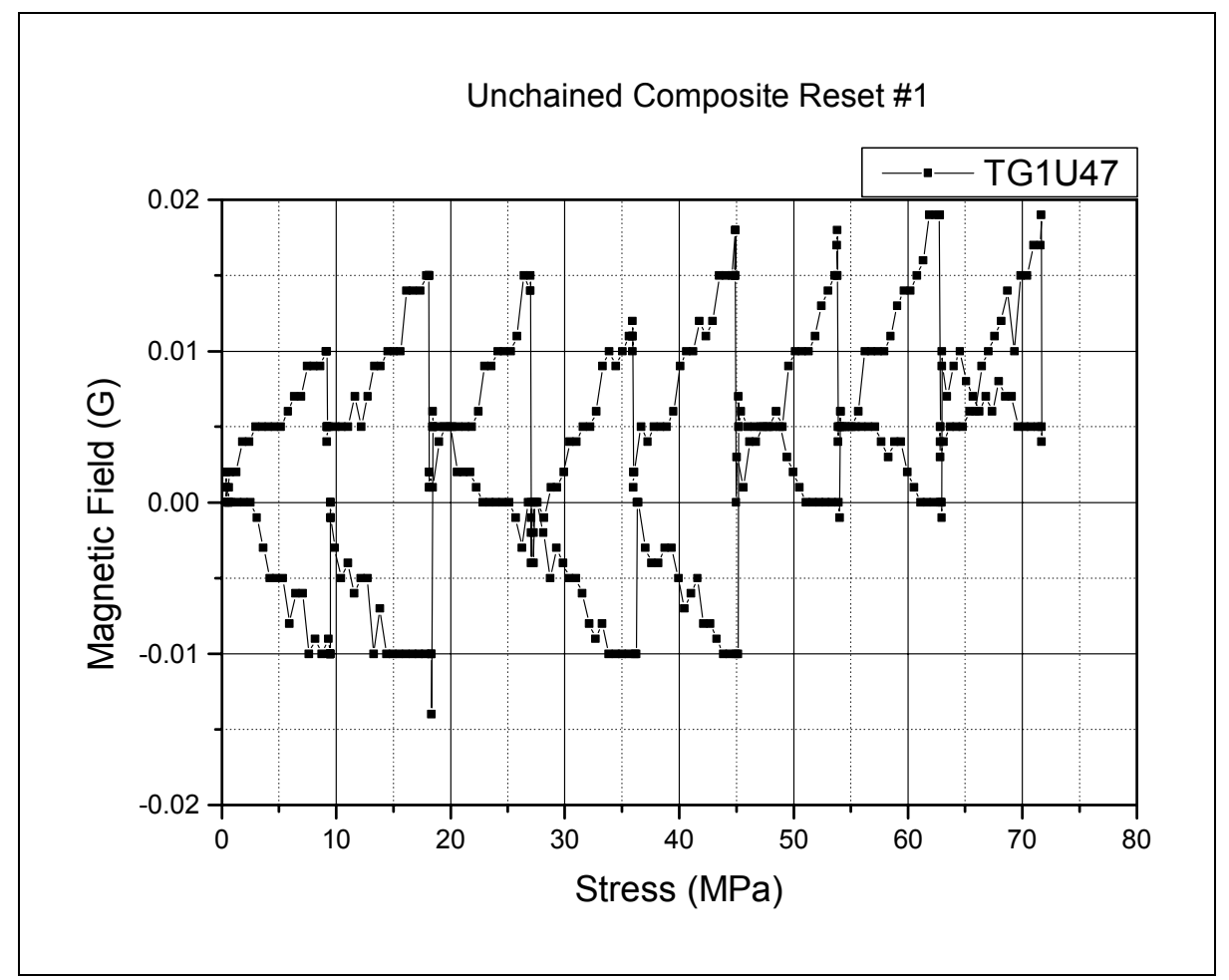

Figure 20. Plot of Reset Protocol 1, unchained composite specimen.

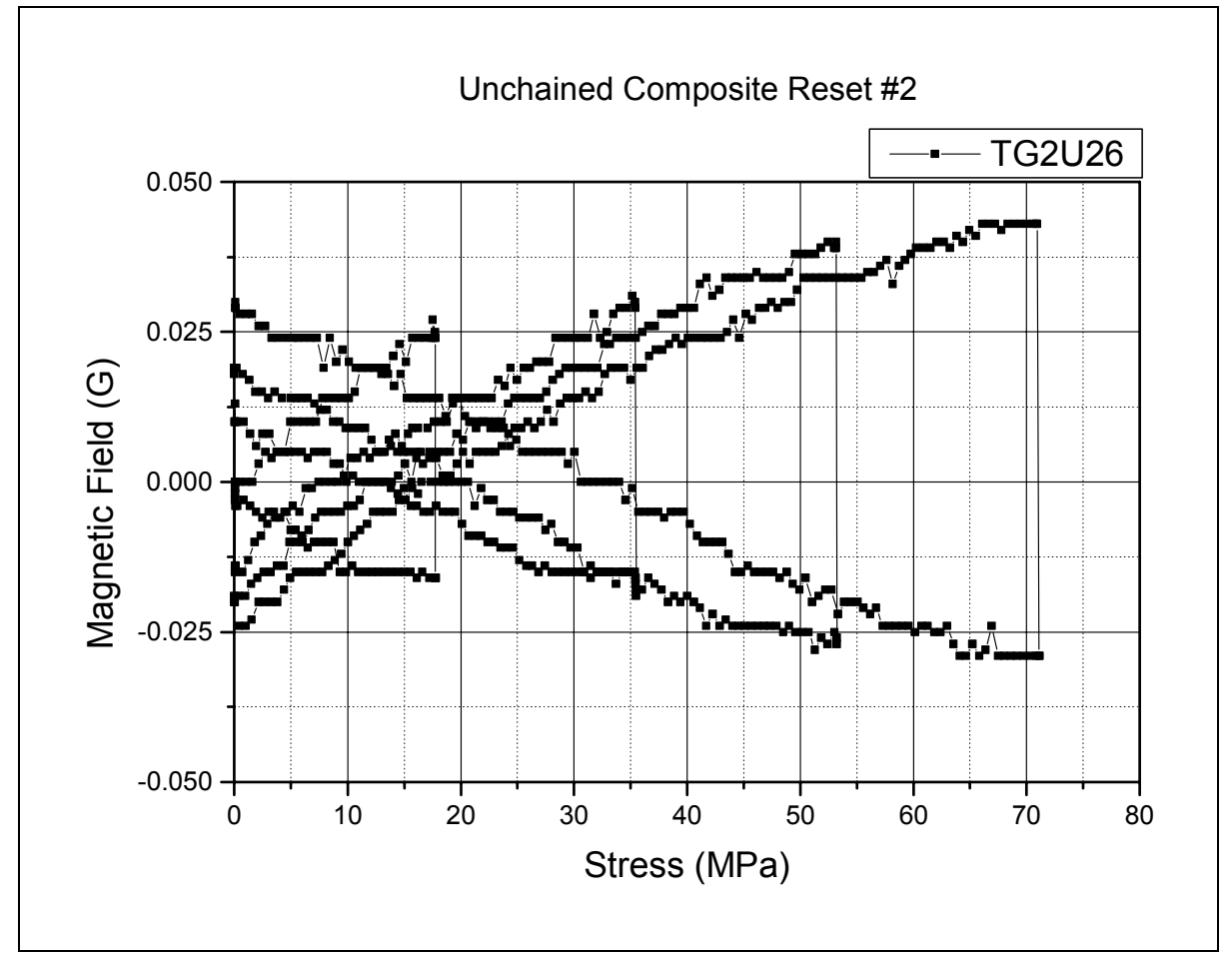

Figure 21. Plot of Reset Protocol 2, unchained composite specimen. 


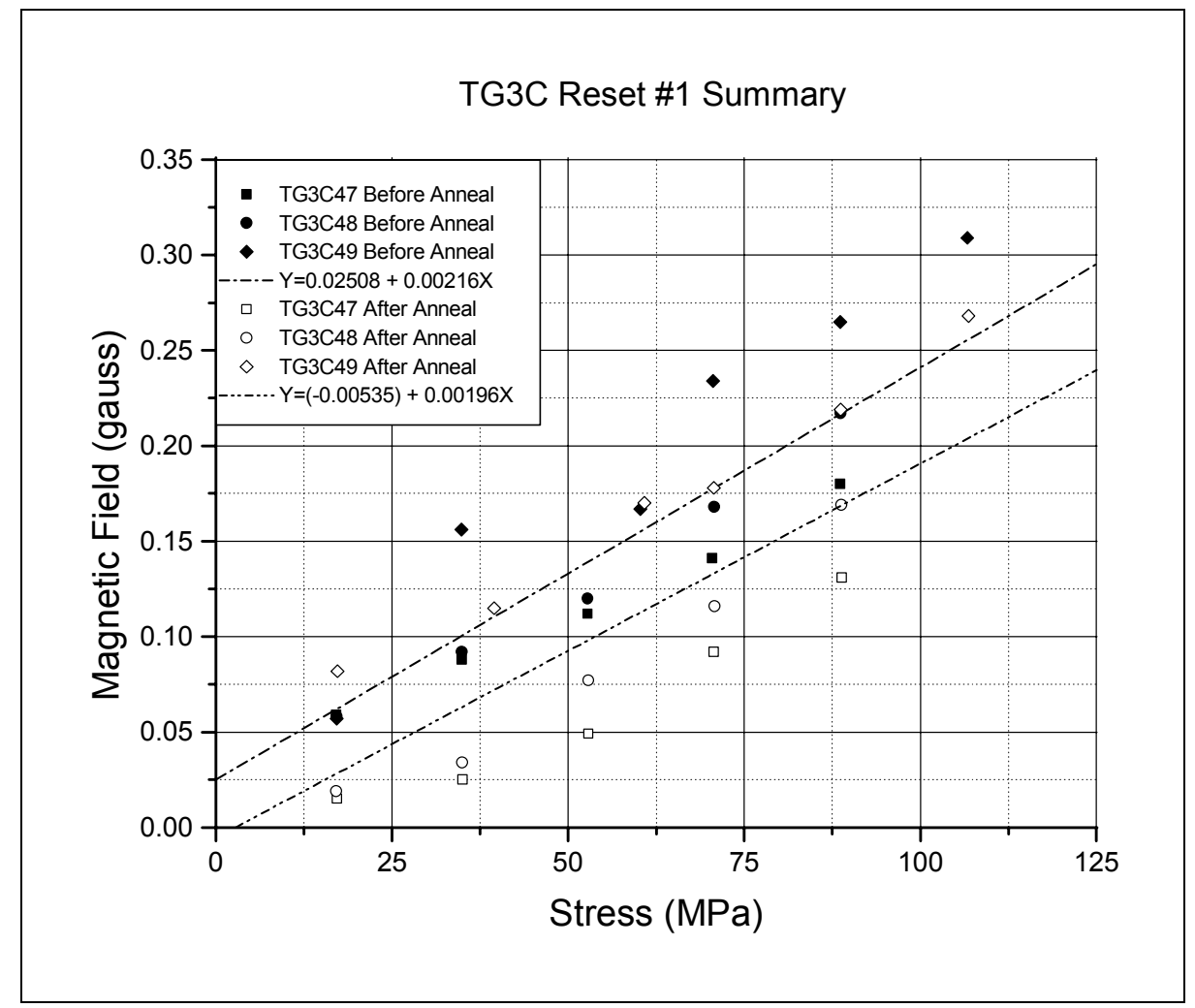

Figure 22. Summary plot for Reset Protocol 1, chained composite specimen.

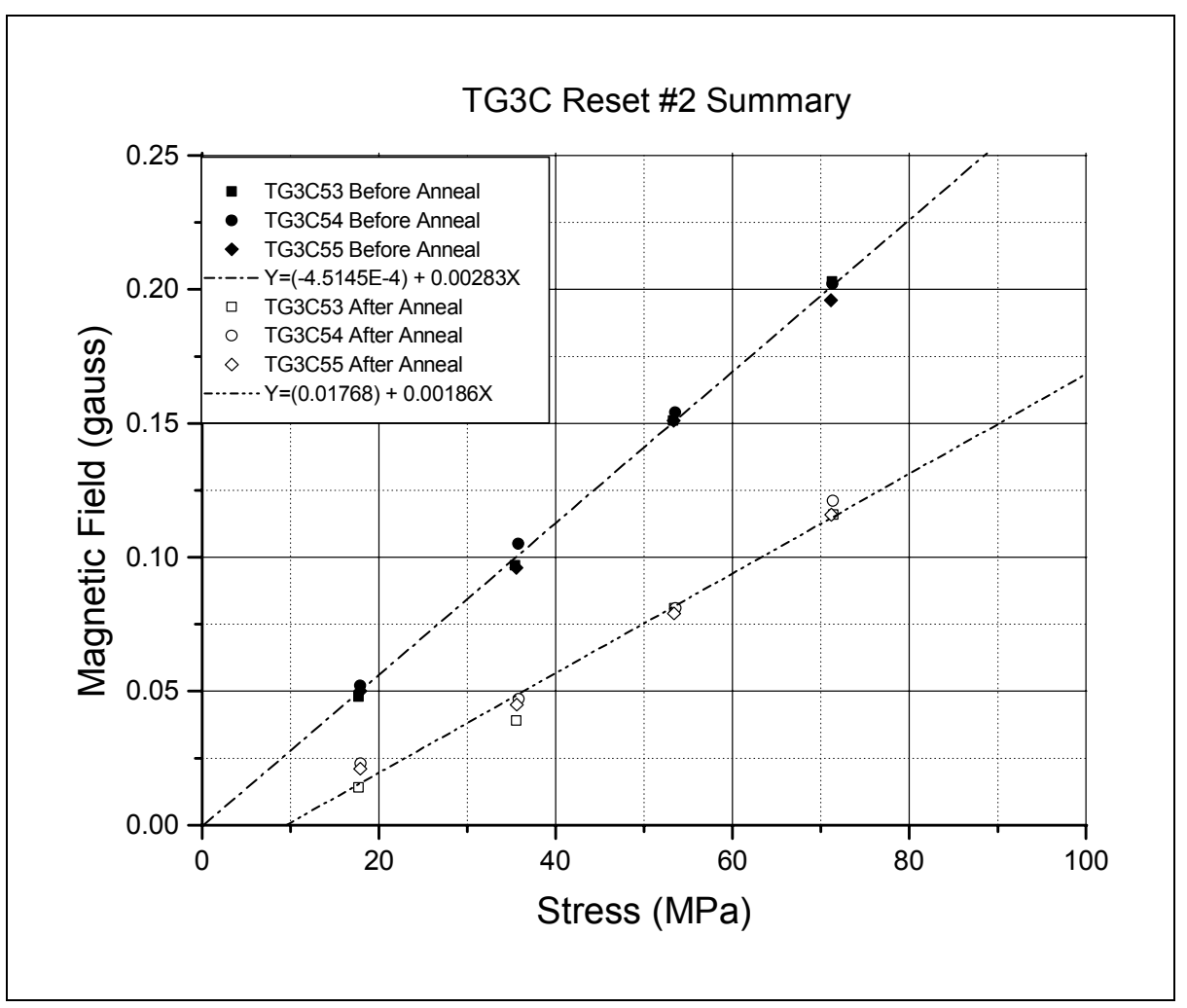

Figure 23. Summary plot for Reset Protocol 2, chained composite specimen. 


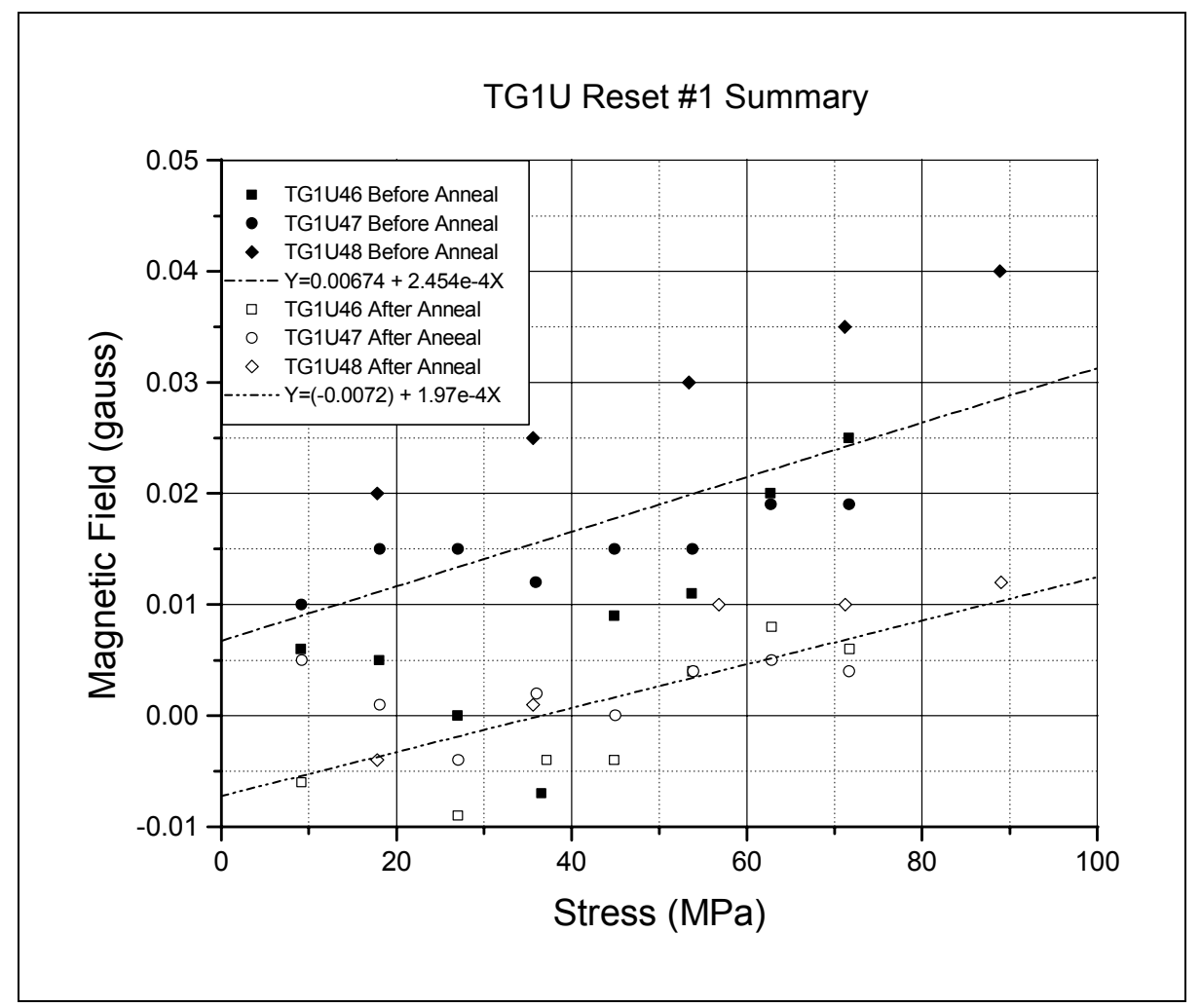

Figure 24. Summary plot for Reset Protocol 1, unchained composite specimen.

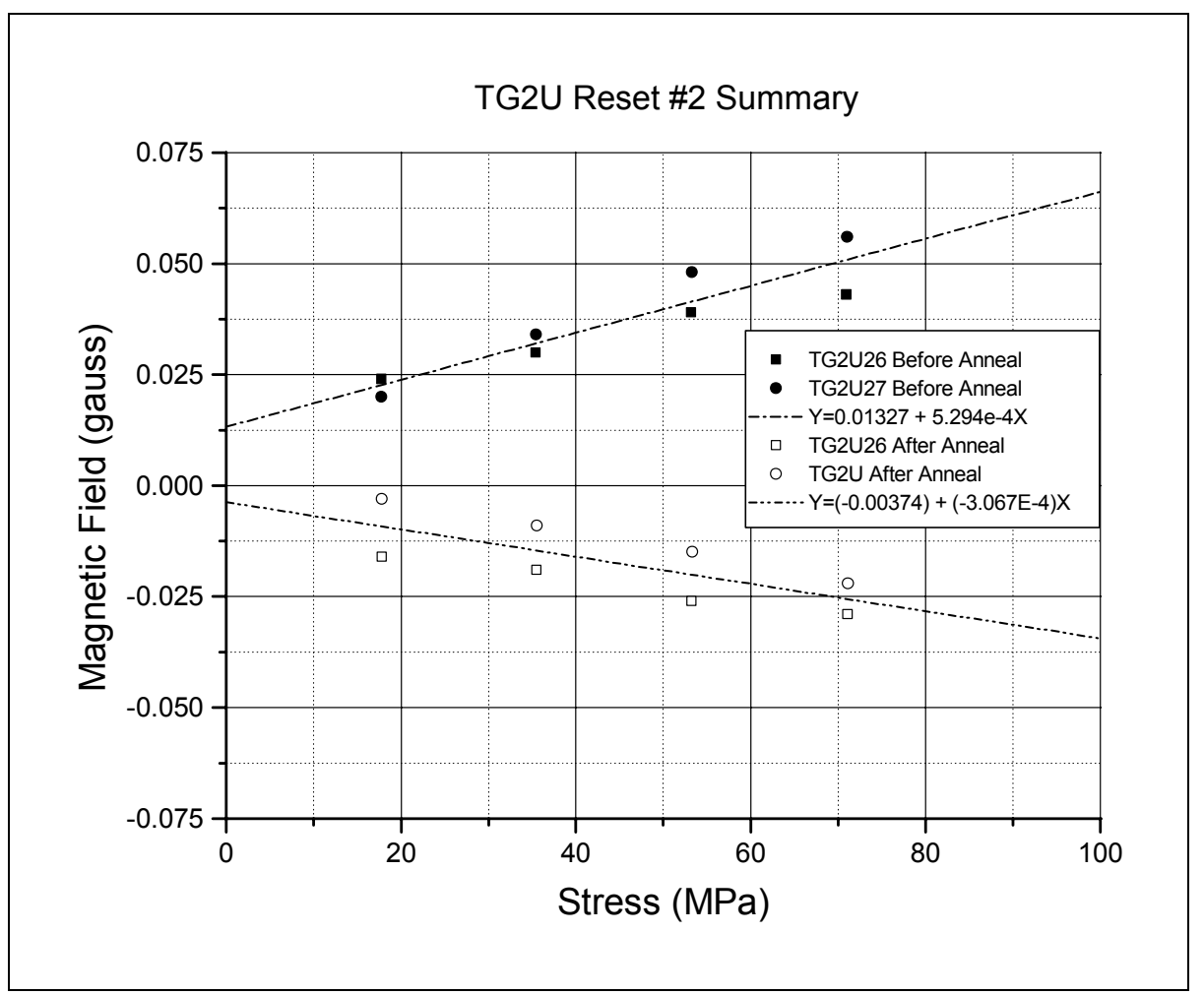

Figure 25. Summary plot for Reset Protocol 2, unchained composite specimen. 


\section{Flexural Response of Tagged Composite Specimen}

\section{Overview of Flexural Testing Program}

Previous investigations (White et al. 1996, 1998, 1999; Quattrone et al. 1998) have used axially loaded magnetostrictive-tagged composite specimens. The present investigation set forth in a previously unexplored direction: the investigation of the bending (flexural) response of magnetostrictive-tagged composites.

A bending beam specimen was designed and fabricated at the University of South Carolina to be representative of the type of F RP composite materials used in construction engineering applications. The specimen was intended for portability and hands-on experimental demonstration of magnetostrictive-tagged composite properties. The design process used the strain vs magnetic field coefficients to estimate the specimen dimensions that would produce optimal response under certain loading conditions (Giurgiutiu et al. 1999). For assumed Young's modulus $\mathrm{E}_{11}=8 \mathrm{GPa}$ and flexural strength $\mathrm{S}_{\mathrm{F}}=150 \mathrm{MPa}$, a specimen design of $1000 \mathrm{~mm}$ long, $100 \mathrm{~mm}$ wide, and $6.5 \mathrm{~mm}$ thick was used. The specimen was fabricated by Reichhold Chemicals (Research Triangle Park, NC) from a glass-fiber combination of 5x4 fiber and fill woven roving 36 oz/sq yd, 8 layers, with Atlac 580-05 urethane-modified vinyl ester resin. The resin-to-glass ratio was 1:1. An MEKP initiator (1\%) produced a room-temperature cure in $90 \mathrm{~min}$ utes. Magnetostrictive powder was used only in the two outside layers and only in the middle $500 \mathrm{~mm}$ of the $1000 \mathrm{~mm}$ span, with a $25 \%$ weight fraction. After fabrication, the specimen was trimmed with a band saw. Selvage was kept for further evaluation of terfenol-D particle distribution in the bending beam material.

\section{Experimental Setup}

A list of equipment used in the experiment is given in Table 4. This equipment permitted the simultaneous measurement of beam deflection, mechanical strains, and magnetic response of the tagged beam. The tagged composite beam 
was supported on concrete blocks (500 mm equivalent span) and loaded gradually with an incremental number of clay bricks ( $2 \mathrm{kgf}=19.6 \mathrm{~N}$ each). Strain gages were placed on both the upper and lower surfaces of the specimen at midspan and were connected in a half-bridge configuration to the strain indicator. The magnetic field produced by the magnetostrictive particles was collected via the gaussmeter. A special fixture was constructed to ensure proper and repetitive alignment of the gaussmeter probe with respect to the composite surface.

Table 4. List of equipment used in flexural testing of tagged composite beam experiment.

\begin{tabular}{lll}
\hline Name & Model & Manufacturer \\
\hline Strain Gages & CEA-06-125UT-120 & Measurement Group, Inc. \\
Strain Indicator & P-3500 & Measurement Group, Inc. \\
Gaussmeter & Model 450 & Lakeshore Cryotonics, Inc. \\
LVDT displacement transducer & B-50 & Solartron Co. \\
SCXI amplifier unit & SCXI-1000 & National Instruments Co. \\
LabView & NI Professional Measurement Suite & National Instruments Co. \\
PCMCIA card & DAQcard-Al-16E-4 16 channel & National Instruments Co. \\
Composite material & & Reichhold Chemicals \\
Permanent Magnets & 6"X4"x1" Ceramic 8 Magnet & Adams Magnetic Products Co. \\
\hline
\end{tabular}

For correlation purposes, midspan displacement was also measured. A linear variable displacement transducer (LVDT) and a nonmagnetic (aluminum and brass) clamping fixture were used. Details of the midspan instrumentation are shown in Figure 26. Initial trials showed that the strain gauge and LVDT electromagnetic fields do not influence the gaussmeter reading of the magnetostrictively induced magnetic field.

The experimental data were collected with a National Instruments SCXI unit and data acquisition card in a standard personal computer. A custom data acquisition program was written for this application using LabView, a software package developed by maker of the data acquisition hardware. After proper calibration of all system components, magnetic field, strain, and deflection data were acquired through this software and then processed in Microsoft Excel ${ }^{\circledast}$. 


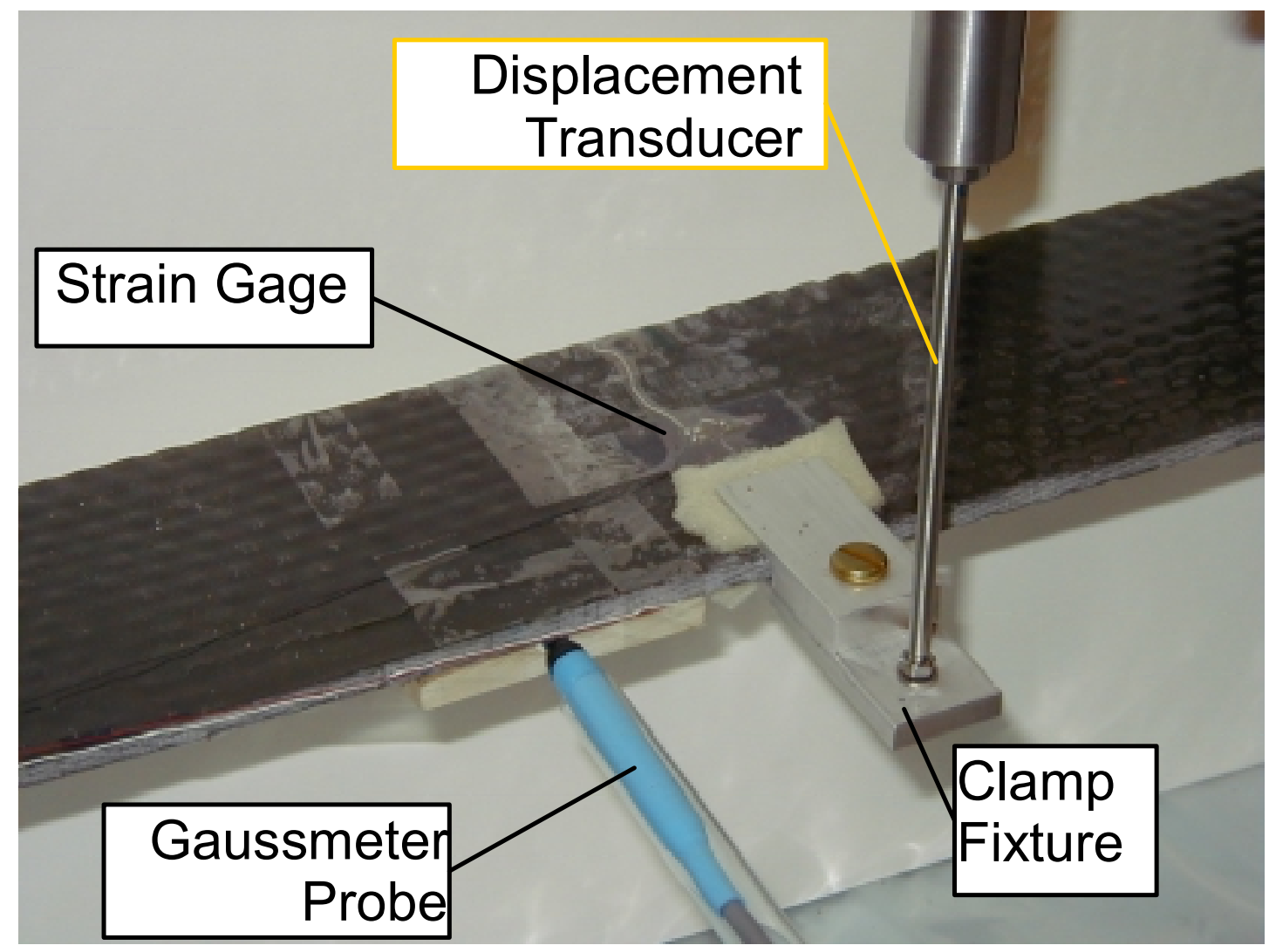

Figure 26. Gaussmeter probe, strain gage, and displacement transducer on the magnetostrictive composite beam.

\section{Effects of Annealing on Flexural Response}

The results presented here refer to flexural response experiments both with and without magnetic annealing between loading cycles. The magnetic response of the specimen without annealing between cycles is lower in amplitude but has very small hysteresis. The response of the specimen with annealing between loading cycles is of higher amplitude, but has very pronounced hysteresis. Both experiments (i.e., with and without annealing between cycles) were conducted in a similar fashion. The tagged composite beam was simply supported on two concrete blocks and was gradually loaded with 3 bricks. The loading sequence, quantified in terms of number of applied bricks, was: $0,1,2,3,2,1,0$. Displacement, strain, and magnetic field data were taken for each constant load level. Ten loading/unloading cycles were performed. In the first experiment, no magnetic annealing was applied between cycles. In the second experiment, magnetic annealing (740 gauss for 1.5 minutes, on average) was applied between each loading cycle using a pair of strong permanent magnets. 
The magnetic field response of the un-annealed specimen during loading is given in Figure 27a. At the time of performing the experiment and acquiring data, the specimen had not been anneal ed for more than 2 days. Thus, the results are indicative of the response of a field-deployed magnetostrictive-tagged composite material. The small difference between readings from different cycles illustrates the deviation expected from the use of general-purpose magnetic measurement equipment. Mean value plots and standard deviation error bars of the magnetic field response are presented in Figure 27b. It can be seen that the magnetic field response is virtually linear. Of interest is also the small value of hysteresis displayed by these measurements.

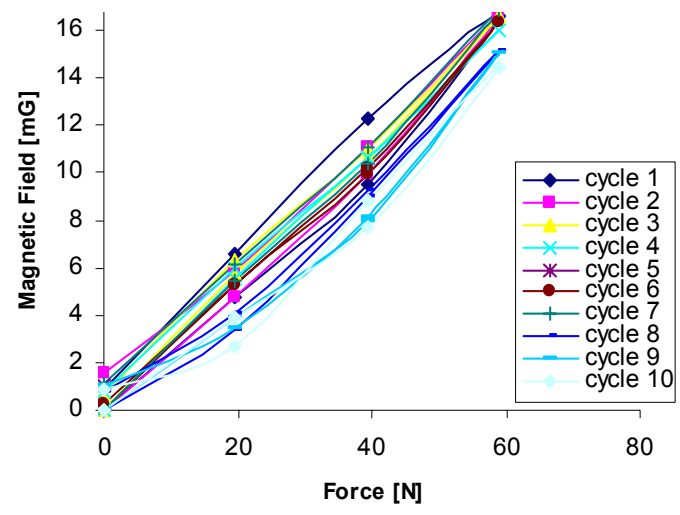

(a)

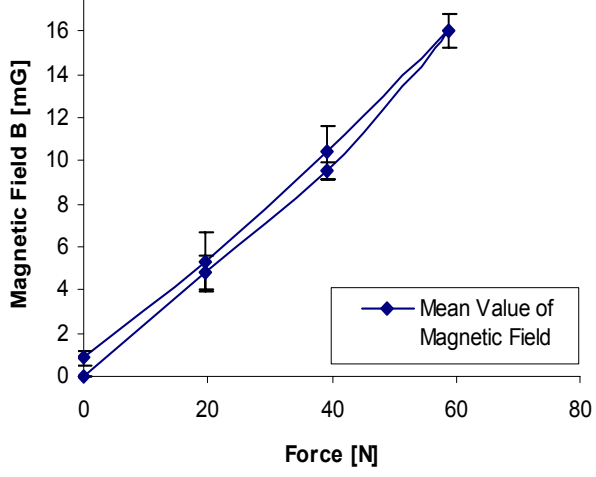

(b)

Figure 27. Magnetic field vs applied force for specimen without annealing between cycles: (a) original data for 10 loading cycles; (b) mean value and standard deviation.

These observations indicate that non-annealed magnetostrictive-tagged composites in bending present satisfactory repeatability and small hysteresis. Similar observations were made by White and Brouwers (1998) for axially loaded specimens and axially measured magnetic fields.

Statistical processing of data in Figure 28a yielded the mean and standard deviation data presented in Figure 28b. From these data, a value of the strainmagnetic field coefficient for un-annealed tagged FRP composites in bending could be determined. The obtained value, $C_{\varepsilon}=12.03$ gauss $/(\mathrm{m} / \mathrm{m})$, is about onethird the $35.74 \mathrm{gauss} /(\mathrm{m} / \mathrm{m})$ for the composite samples tested in tension by Trovillion et al. (1999). 


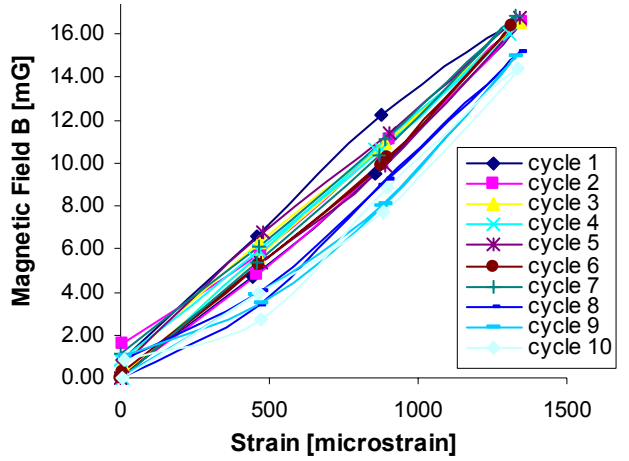

(a)

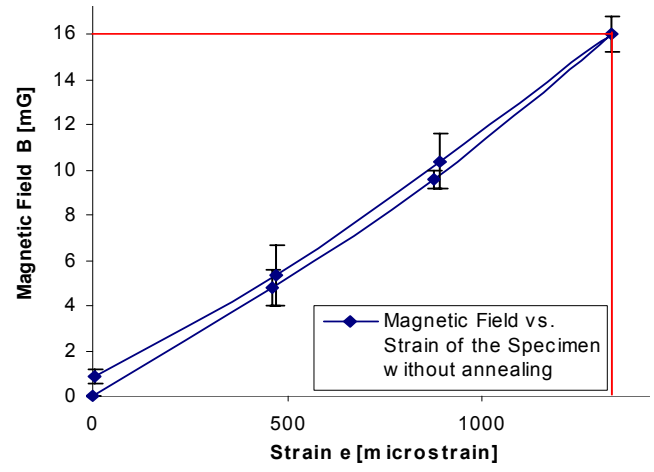

(b)

Figure 28. Magnetic field vs strain for specimen without annealing between cycles:

(a) original data for 10 loading cycles; (b) mean value and standard deviation.

The procedure for the experiment with annealing is similar to that for the experiment without annealing, and is presented in full detail in Giurgiutiu et al. 1999. The magnetic field response with annealing between cycles is presented in Figure 29a. As in the previous experiment, the small difference between readings from different cycles illustrates the deviation to be expected from the use of general-purpose magnetic measurement equipment.

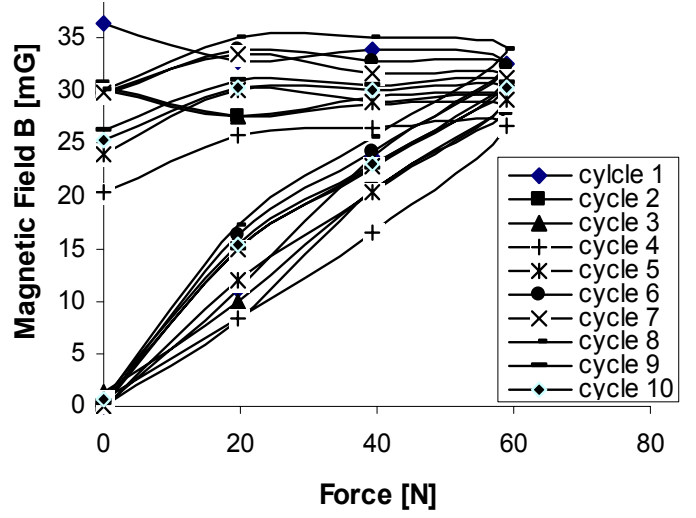

(a)

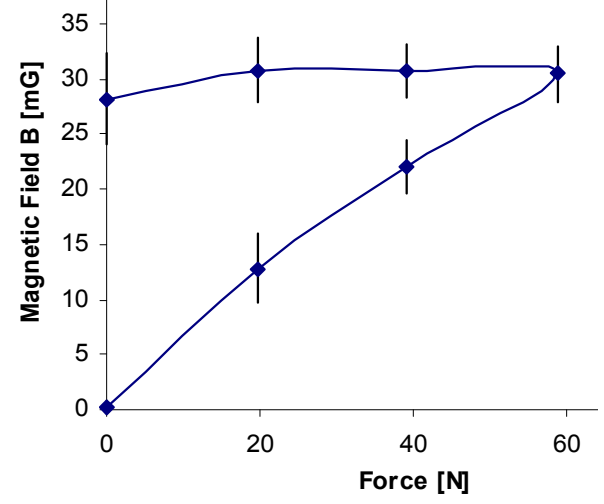

(b)

Figure 29. Magnetic field vs force for specimen with annealing between cycles: (a) original data for $\mathbf{1 0}$ loading cycles; (b) mean value and standard deviation.

Mean value plots and standard deviation error bars of the magnetic field re sponse are presented in Figure 29b. It can be seen that, on the increasing branch of the curve, the magnetic field response is virtually linear. On the decreasing branch of the curve the marginal response is very small and significant hysteresis is present. These observations indicate that annealed magnetostrictive-tagged composites possess a higher initial response but also a very pronounced hysteresis. Similar observations were made by White and Brouwers (1998) for axially loaded magnetostrictive-tagged composite specimens with an axially measured magnetic field. 
Using the recorded strain-applied force data and the magnetic field-applied force data (Figure 29a), the magnetic field-strain relationship was developed (Figure 30a). Then, statistical processing of the data in Figure 30a yielded the mean and standard deviation plot presented in Figure 30b. From this plot, a value for the strain-magnetic field coefficient for annealed magnetostrictive-tagged FRP composites in bending could be determined. The obtained value of $C_{\varepsilon}=16.8$ gauss $/(\mathrm{m} / \mathrm{m})$ is 28.4 percent higher than the 12.03 gauss $/(\mathrm{m} / \mathrm{m})$ value obtained from the nonannealed tests on the same bending specimen.

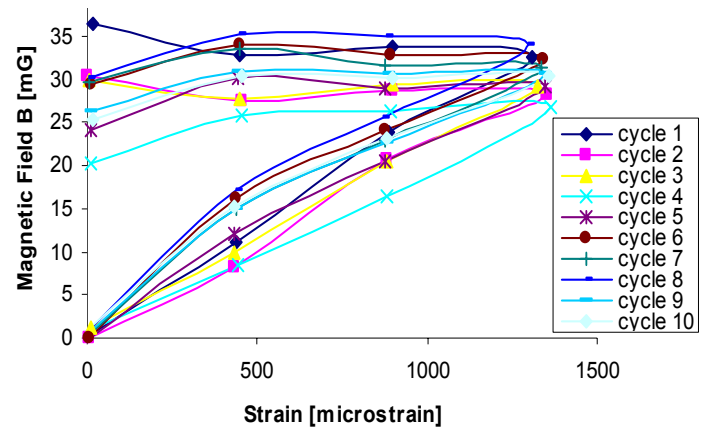

(a)

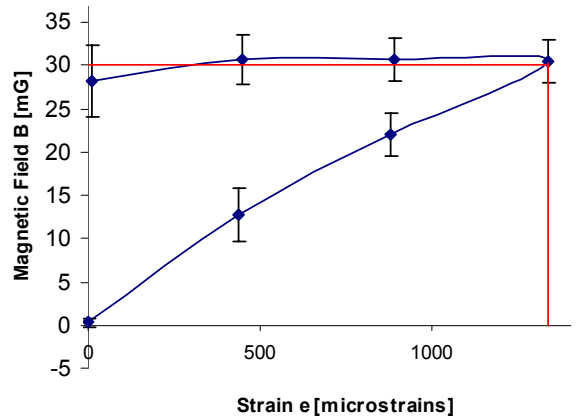

(b)

Figure 30. Magnetic field vs strain for specimen with annealing between cycles: (a) original data for $\mathbf{1 0}$ loading cycles; (b) mean value and standard deviation.

\section{Summary}

Comparison of present bending results with previously published results for axial loading indicate that, for the bending specimen, the coefficient for the annealed test was 28.4 percent higher than the coefficient for the unannealed test [16.8 gauss $/(\mathrm{m} / \mathrm{m})$ for bending vs 12.03 gauss $/(\mathrm{m} / \mathrm{m}$ ) for axial load]. This is what was expected on the basis of previous work (Quattrone, Berman, and White 1998). A comparison of the annealed data for the composite in bending and that for the composite in tension showed that the bending specimen had a coefficient about one-third that of the tension specimen $[12.03$ gauss $/(\mathrm{m} / \mathrm{m})$ for the bending specimen vs 35.74 gauss $/(\mathrm{m} / \mathrm{m})$ for the tension specimen]. This difference could be due to the non-uniform stress distribution through the thickness of the bending specimen or due to the difference in fiber geometry and architecture between the two specimens. 


\section{Damage Detection in Magnetostrictive- Tagged Composites}

\section{Experimental Procedures}

This chapter presents the results of damage detection experiments performed on six glass-reinforced polyester composite specimens tagged with terfenol-D. The specimens were loaded in uniaxial tension with the test setup discussed in section 2.2 of Li 2000. Maximum load and loading rate were consistent with the procedures used for the undamaged composite specimens as discussed in the procedures section (Li 2000).

\section{Introduction of Damage to the Test Specimens}

After the magnetomechanical response of each composite specimen was characterized at specified points along the axes of the specimen, damage was introduced by drilling holes and cutting square notches through the specimens.

First, a $3.18 \mathrm{~mm}$ diameter hole was drilled through the center of the gage section of three of the specimens. After magnetomechanical characterization the holes were enlarged to $4.76 \mathrm{~mm}$. Again, the magnetic fluxes at each grid position were measured. The holes were then enlarged to $6.35 \mathrm{~mm}$ and the process was repeated.

Next, square notches were cut into the side of the other three specimens at the center of the gage section. The notches were $1 \mathrm{~mm}$ in width, and were cut from the left edge to the desired position. For the first test in this series, the notches were cut to one-sixth of the specimen width (a notch length of $3.2 \mathrm{~mm}$ ). After magnetic fluxes were measured under load the notches were extended to onethird of the specimen width (or a notch length of $6.0 \mathrm{~mm}$ ) and the process repeated. Figure 31 shows both types of damage specimens and their geometry. 


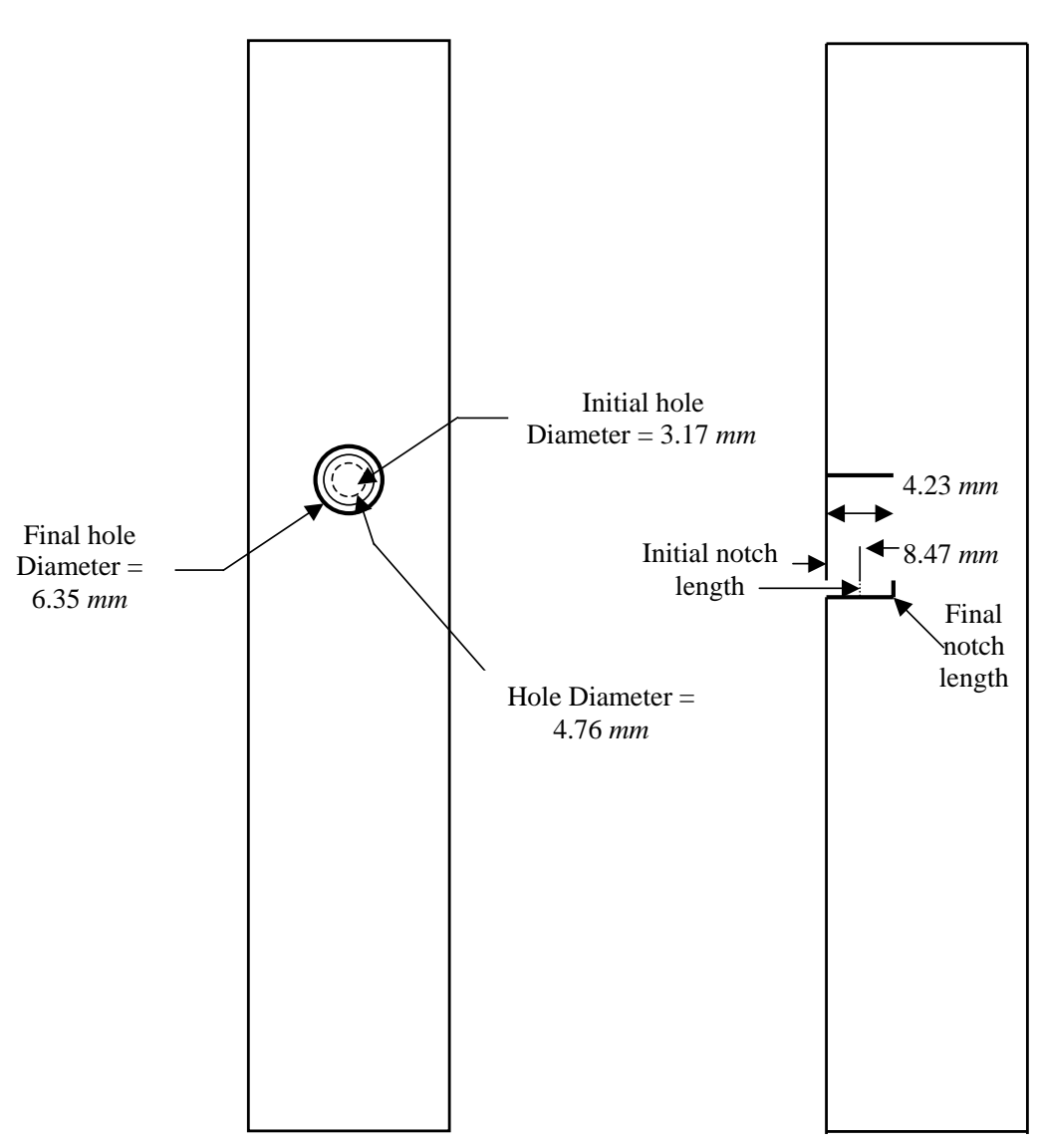

(a)

(b)

Figure 31. Geometry of damage specimens.

\section{Damage Characterization Results}

\section{Notched Specimens}

Figure $32^{*}$ shows the magnetic flux $\Delta B_{2}$ along the gage length at three crosssection locations for notched specimens after the initial cut to $x_{2}=-6.4 \mathrm{~mm}$. The difference in readings between damaged and undamaged states is shown in Figure 33. Near the notch tip there is an appreciable change in $\Delta B_{2}$ at the center of the gage section $\left(x_{1}=0\right)$. Moving away from the notch tip, $\Delta\left(\Delta B_{2}\right)$ gradually decreases to zero. In addition, the magnetic flux $\Delta B_{2}$ near the notch tip was larger after the notch was introduced.

\footnotetext{
* Figures $32-49$ are presented in sequence at the end of this chapter.
} 
Figure 34 shows the magnetic flux $\Delta B_{2}$ along the centerline of the gage section $\left(x_{1}=0\right)$ and in Figure 35 the difference between damaged and undamaged signals is shown. Again, there is a slight concentration in $\Delta\left(\Delta B_{2}\right)$ approaching the notch tip.

Although the flux readings in the chain direction (see Figures 32 - 35) can be interpreted to yield information about the damaged state of the material, the overall sensitivity to damage appears to be quite small. However, flux readings $\Delta B_{3}$ (magnetic flux in the thickness direction) were found to be extremely sensitive to damage. In Figure 36 the difference in magnetic flux $\Delta\left(\Delta B_{3}\right)$ between damaged and undamaged states is plotted along the gage length at several cross-sectional locations. The data along the line $\left(x_{1}=-6.4 \mathrm{~mm}\right)$ correspond to the notch tip and a very large concentration (about 0.5 gauss) occurs right at the notch tip $\left(x_{1}=0\right)$. In Figure 37 the difference in magnetic flux $\Delta\left(\Delta B_{3}\right)$ along the centerline of the gage section $\left(x_{1}=0\right)$ is plotted. Again, a very strong correlation in signal is shown at the notch tip.

After completing magnetic flux measurements on specimens notched to one-sixth of their width, the notch was extended to one-third of the specimen width. Measurements were repeated to obtain $\Delta B_{2}$ and $\Delta B_{3}$ at grid positions across the surface of the specimens.

Figure 38 shows the difference in $\Delta B_{2}$ between damaged and undamaged specimens as a function of gage length position. Near the notch tip the change in $\Delta B_{2}$ is largest, and then drops gradually moving away from the tip.

Figure 39 shows the difference in $\Delta B_{2}$ between damaged and undamaged specimens along the centerline of the gage section. Again, the largest signals are recorded near the tip of the notch (about 0.15 gauss).

Although the presence of a stress concentration is clearly demonstrated in Figure 39, once again the higher sensitivity is achieved by comparing $\Delta B_{3}$ between damaged and undamaged specimens. In Figure 40 the difference in $\Delta B_{3}$ readings is shown along the gage length at two different cross-sectional locations. Figure 41 shows the difference in $\Delta B_{3}$ along the cross-section at the center of the gage section.

These two figures clearly show a very strong correlation between $\Delta\left(\Delta B_{3}\right)$ and the position of the notch tip. Moving away from the notch tip, $\Delta B_{3}$ drops in magnitude quickly. 
By comparing the results of the one-sixth width notch specimens and one-third width notch specimens it is clear that the change of magnetic fluxes is largest for the one-third width notch specimens in both the $x_{2}$ direction and $x_{3}$ direction. The maximum $\Delta B_{2}$ was observed to be 0.05 gauss at the notch tip in the onesixth width notch specimens, and it reached 0.17 gauss in the one-third width notch specimens. The maximum $\Delta B_{3}$ was measured to be 0.46 gauss at the notch tip in the one-sixth width notch specimens, compared to 0.6 gauss in the one-third width notch specimens. Based on all test results for notched specimens it can be concluded that (1) a magnetic flux concentration is observed near the notch tips and (2) that the magnitude of this concentration increases as the notches are extended.

\section{Through-Hole Damage Results}

Figures 42 and 43 show the difference in $\Delta B_{3}$ along the centerline of the gage section and a gage length for $3.18 \mathrm{~mm}$ diameter through-hole specimens. There is a significant increase in $\Delta\left(\Delta B_{3}\right)$ approaching the edge of the hole (from zero at the specimen edge to 0.2 at the hole edge). One interesting phenomenon was the symmetric nature of the distributions along the gage length at $x_{2}= \pm 1.6 \mathrm{~mm}$. $\Delta\left(\Delta B_{3}\right)$ al ong the gage length at $x_{2}=-1.6 \mathrm{~mm}$ was a mirror image of the ones al ong the gage length at $x_{2}=+1.6 \mathrm{~mm}$.

Figures 44 and 45 show the difference in $\Delta B_{3}$ along the centerline of the gage section and a gage length for $3.18 \mathrm{~mm}$ diameter through-hole specimens. Near the hole edge there is an appreciable change in $\Delta\left(\Delta B_{3}\right)$. Moving away from the notch tip, $\Delta\left(\Delta B_{3}\right)$ gradually decreases to zero.

Figures 46 and 47 show similar results for specimens in which the through-hole was enlarged to $4.76 \mathrm{~mm}$. Now the concentration of $\Delta\left(\Delta B_{3}\right)$ is more obvious near the hole edge (zero at the specimen edge to nearly 0.4 gauss at the hole edge).

Figures 48 and 49 show the results after the through-hole was enlarged to its maximum diameter of $6.35 \mathrm{~mm}$. Again, the concentration in $\Delta\left(\Delta B_{3}\right)$ is accentuated (nearly 0.6 gauss at the edge).

The symmetric distribution in $\Delta\left(\Delta B_{3}\right)$ for $3.18 \mathrm{~mm}$ through-hole specimens was also observed in the $4.76 \mathrm{~mm}$ and $6.35 \mathrm{~mm}$ through-hole specimens. Thus, the difference in $\Delta B_{3}$ along one edge of the through-hole was always a mirror image of the distribution along the other edge. 
By comparing the results for all through-hole specimens it is clear that the difference in magnetic fluxes were largest for $6.35 \mathrm{~mm}$ through-hole specimens and smallest for $3.18 \mathrm{~mm}$ through-hole specimens. The maximum $\Delta\left(\Delta B_{3}\right)$ was measured to be 0.2 gauss at the hole edge in $3.18 \mathrm{~mm}$ through-hole specimens, and was 0.4 gauss in $6.35 \mathrm{~mm}$ through-hole specimens. Based on these results it can be concluded that for through-hole specimens a magnetic flux concentration occurs near the hole edge, and the magnitude of the flux increases as the hole is enlarged.

Square notches and through-holes are two very different forms of damage, but very similar results were obtained for both. In both types of damage a magnetic flux concentration was observed near the edge of the damage, and this concentration increased as the severity of the damage (notch length or hole size) increased.

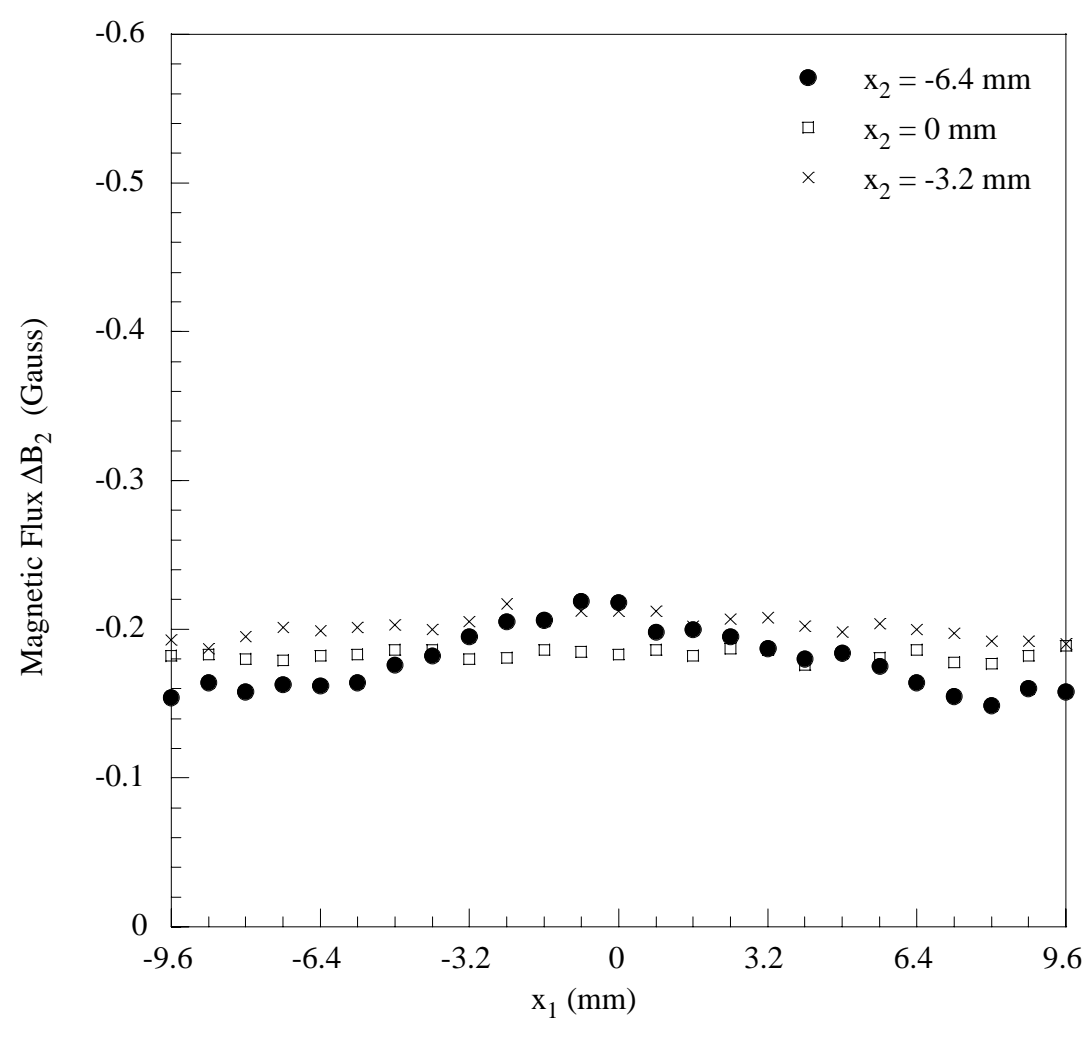

Figure 32. Change in magnetic flux in the chain direction at $\sigma_{1}=24 \mathrm{MPa}$ for notched terfenol-D FRP specimens with notch tip positioned at $x_{2}=-6.4 \mathrm{~mm}$. 


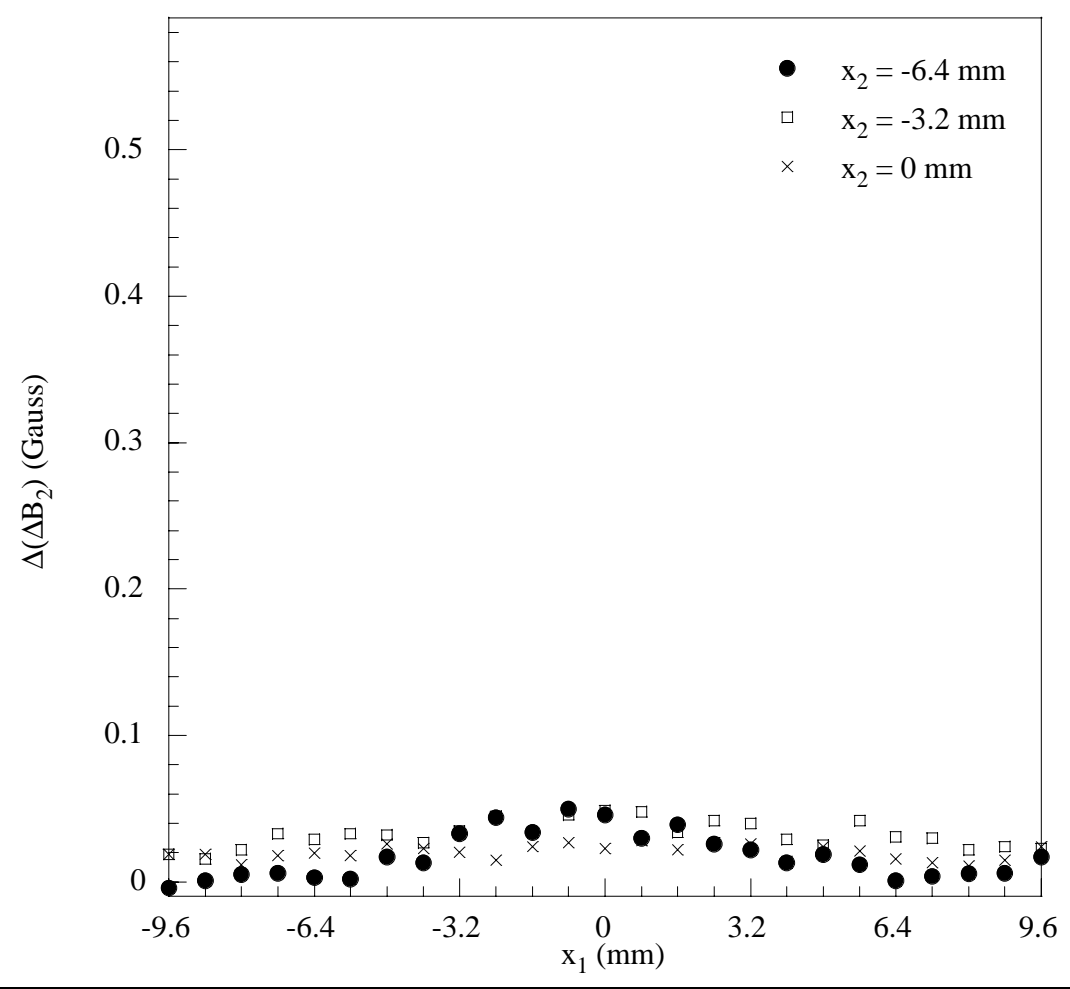

Figure 33. Difference between damaged (notched) and undamaged $\Delta B_{2}$ readings for terfenol-D FRP specimens at $\sigma_{1}=24 \mathrm{MPa}$ with notch tip positioned at $\mathrm{x}_{2}=-6.4 \mathrm{~mm}$.

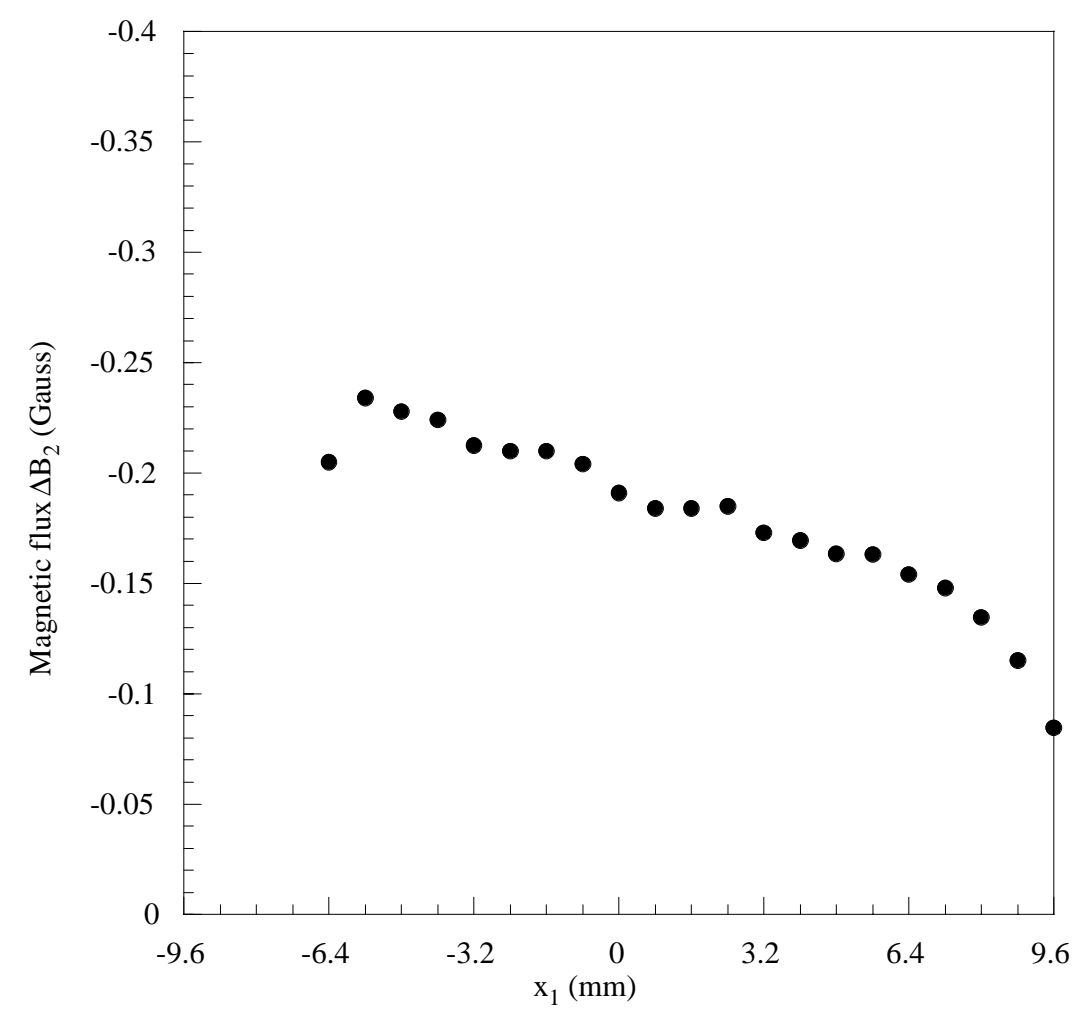

Figure 34. Change in magnetic flux $\Delta \mathrm{B}_{2} \sigma_{1}=24 \mathrm{MPa}$ for notched terfenol-D FRP specimens with notch tip positioned at $\mathrm{x}_{2}=-6.4 \mathrm{~mm}$. 


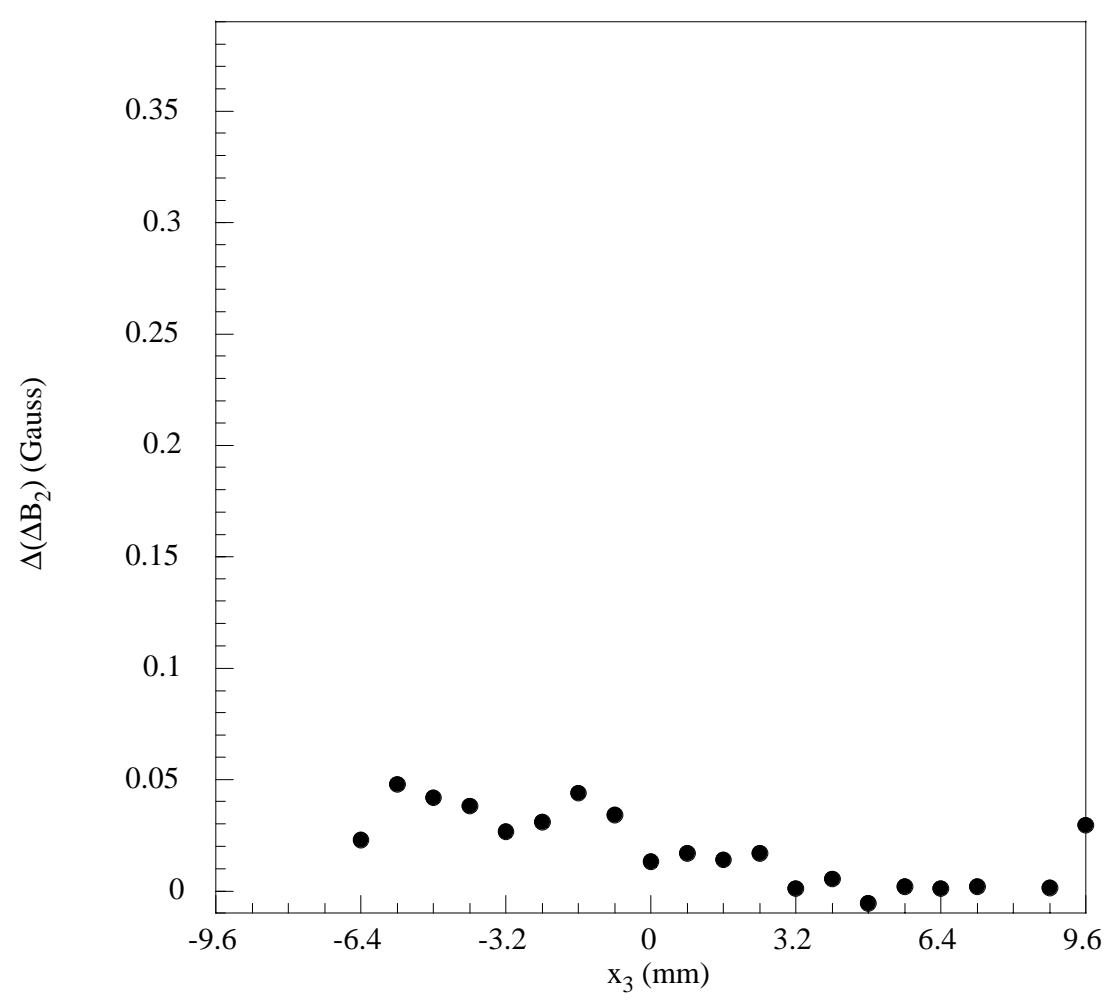

Figure 35. Difference between damaged (notched) and undamaged $\Delta B_{2}$ readings for terfenol-D FRP specimens at $\sigma_{1}=24 \mathrm{MPa}$ with notch tip positioned at $x_{2}=-6.4 \mathrm{~mm}$.

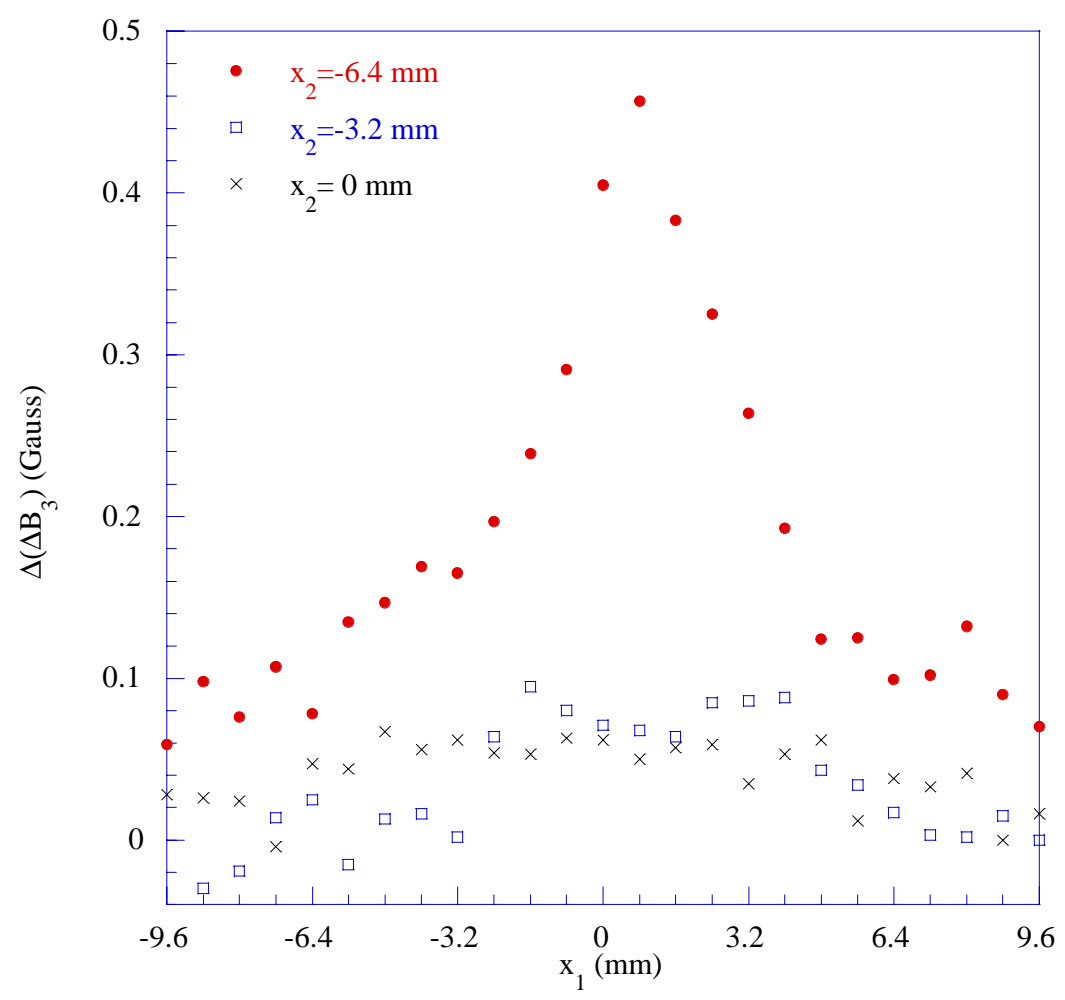

Figure 36. Difference between damaged (notched) and undamaged $\Delta B_{3}$ readings for terfenol-D FRP specimens at $\sigma_{1}=24 \mathrm{MPa}$ with notch tip positioned at $\mathrm{x}_{2}=-6.4 \mathrm{~mm}$. 


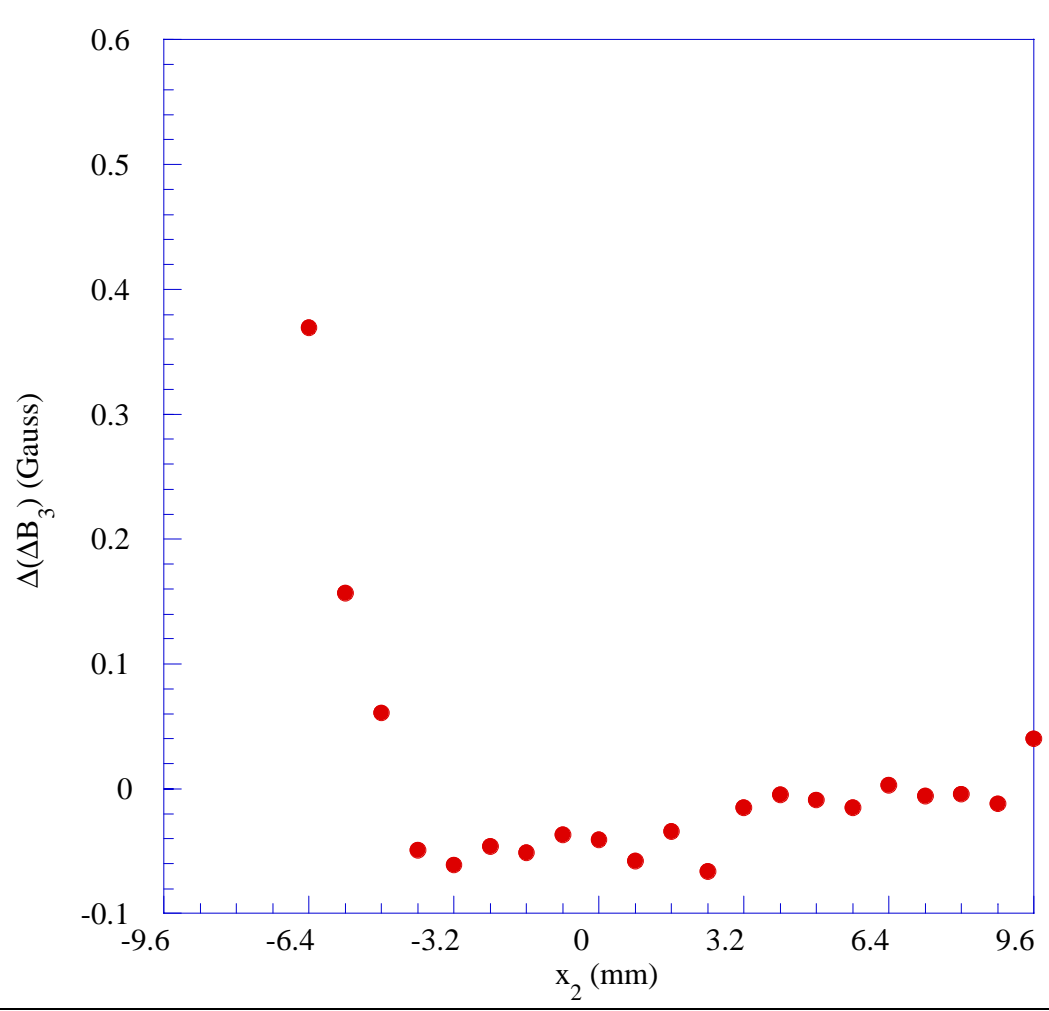

Figure 37. Difference between damaged (notched) and undamaged $\Delta B_{3}$ readings for terfenol-D FRP specimens at $\sigma_{1}=24 \mathrm{MPa}$ with notch tip positioned at $\mathrm{x}_{2}=-6.4 \mathrm{~mm}$.

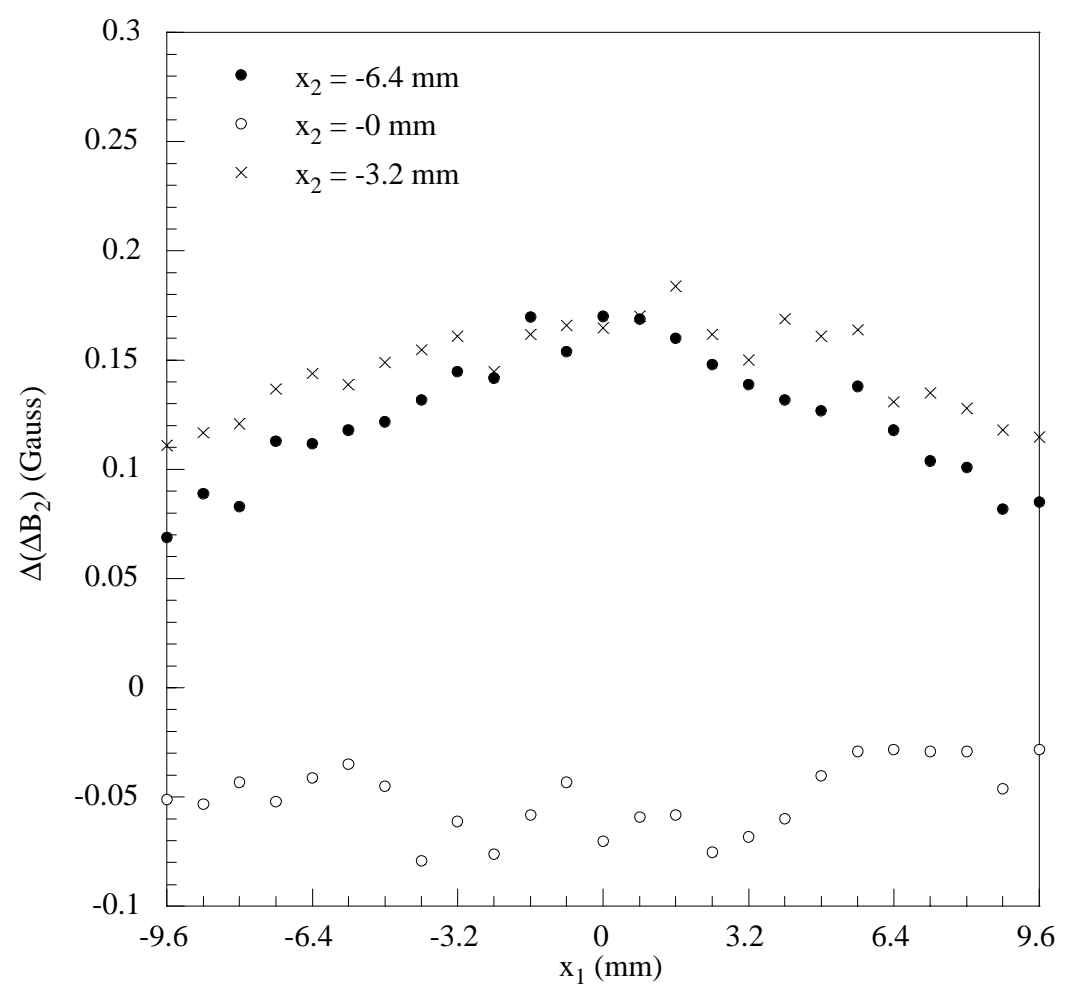

Figure 38. Difference between damaged (notched) and undamaged $\Delta B_{2}$ readings for terfenol-D FRP specimens at $\sigma_{1}=24 \mathrm{MPa}$ with notch tip positioned at $\mathrm{x}_{2}=-3.2 \mathrm{~mm}$. 


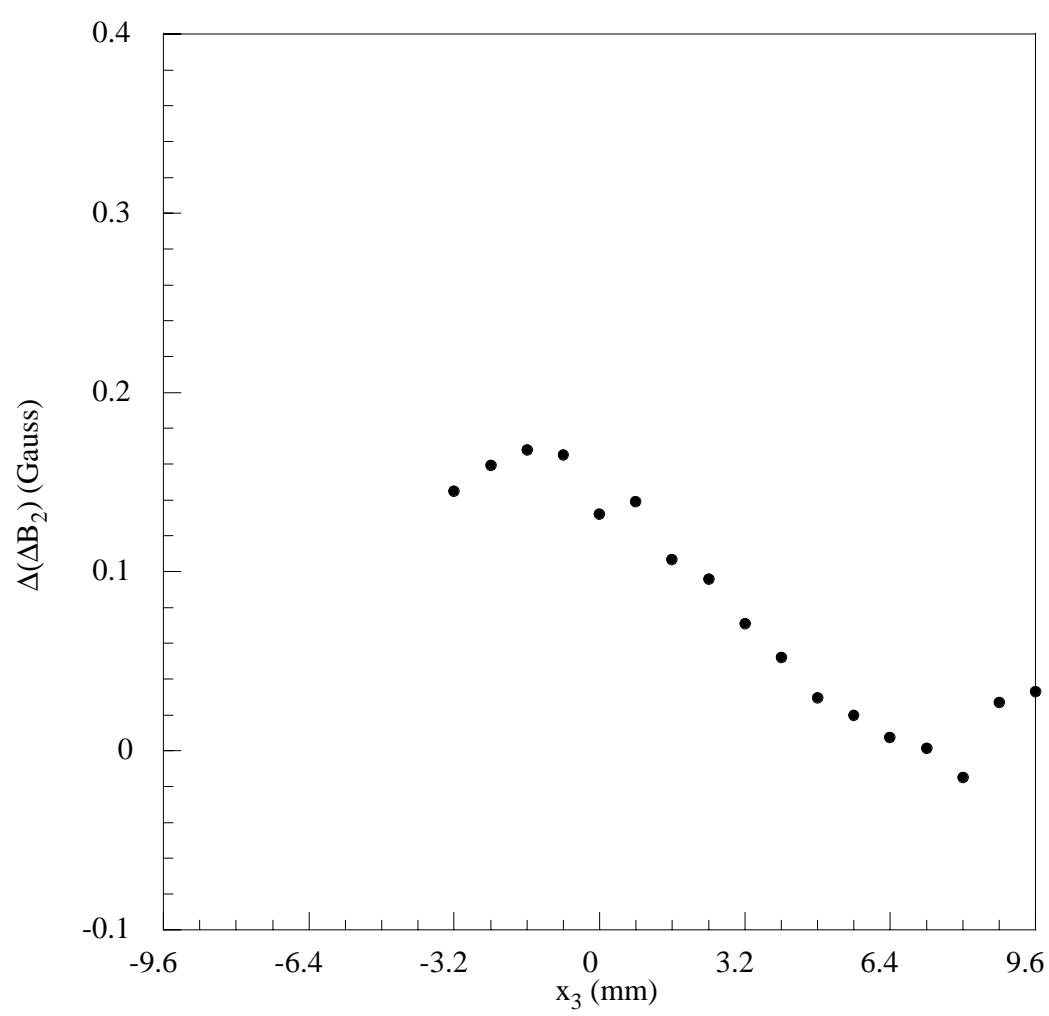

Figure 39. Difference between damaged (notched) and undamaged $\Delta B_{2}$ readings for terfenol-D FRP specimens at $\sigma_{1}=24 \mathrm{MPa}$ with notch tip positioned at $\mathrm{x}_{2}=-3.2 \mathrm{~mm}$.

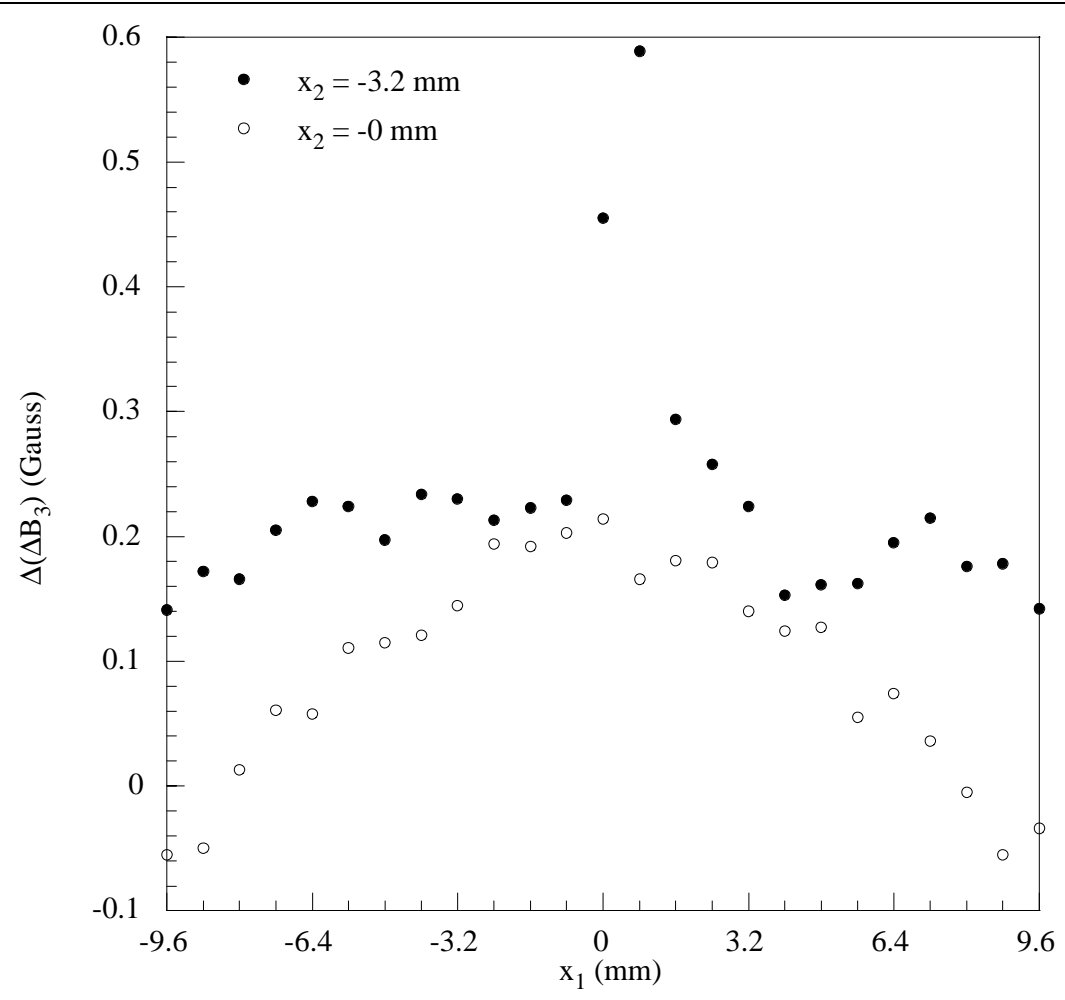

Figure 40. Difference between damaged (notched) and undamaged $\Delta B_{3}$ readings for terfenol-D FRP specimens at $\sigma_{1}=24 \mathrm{MPa}$ with notch tip positioned at $\mathrm{x}_{2}=-3.2 \mathrm{~mm}$. 


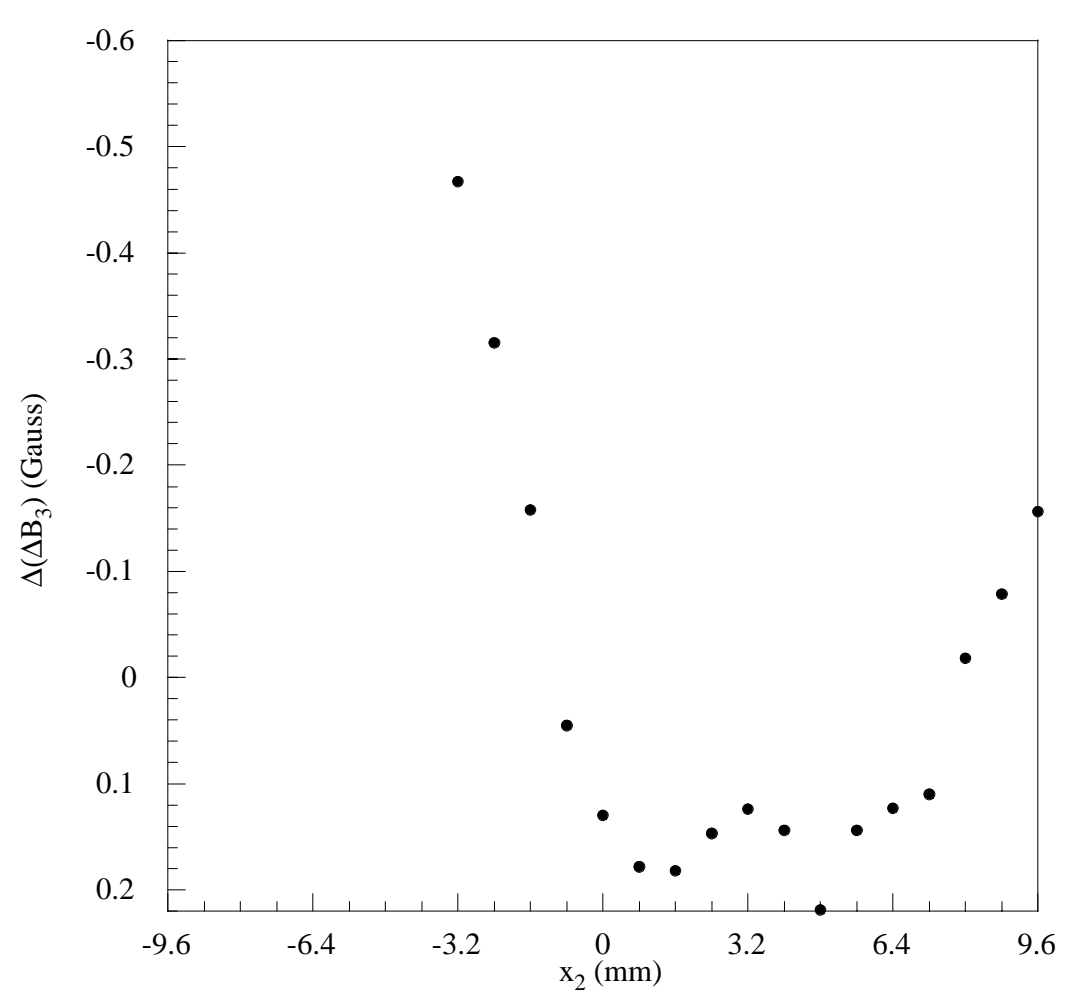

Figure 41. Difference between damaged (notched) and undamaged $\Delta B_{3}$ readings for terfenol-D FRP specimens at $\sigma_{1}=24 \mathrm{MPa}$ with notch tip positioned at $\mathrm{X}_{2}=-3.2 \mathrm{~mm}$.

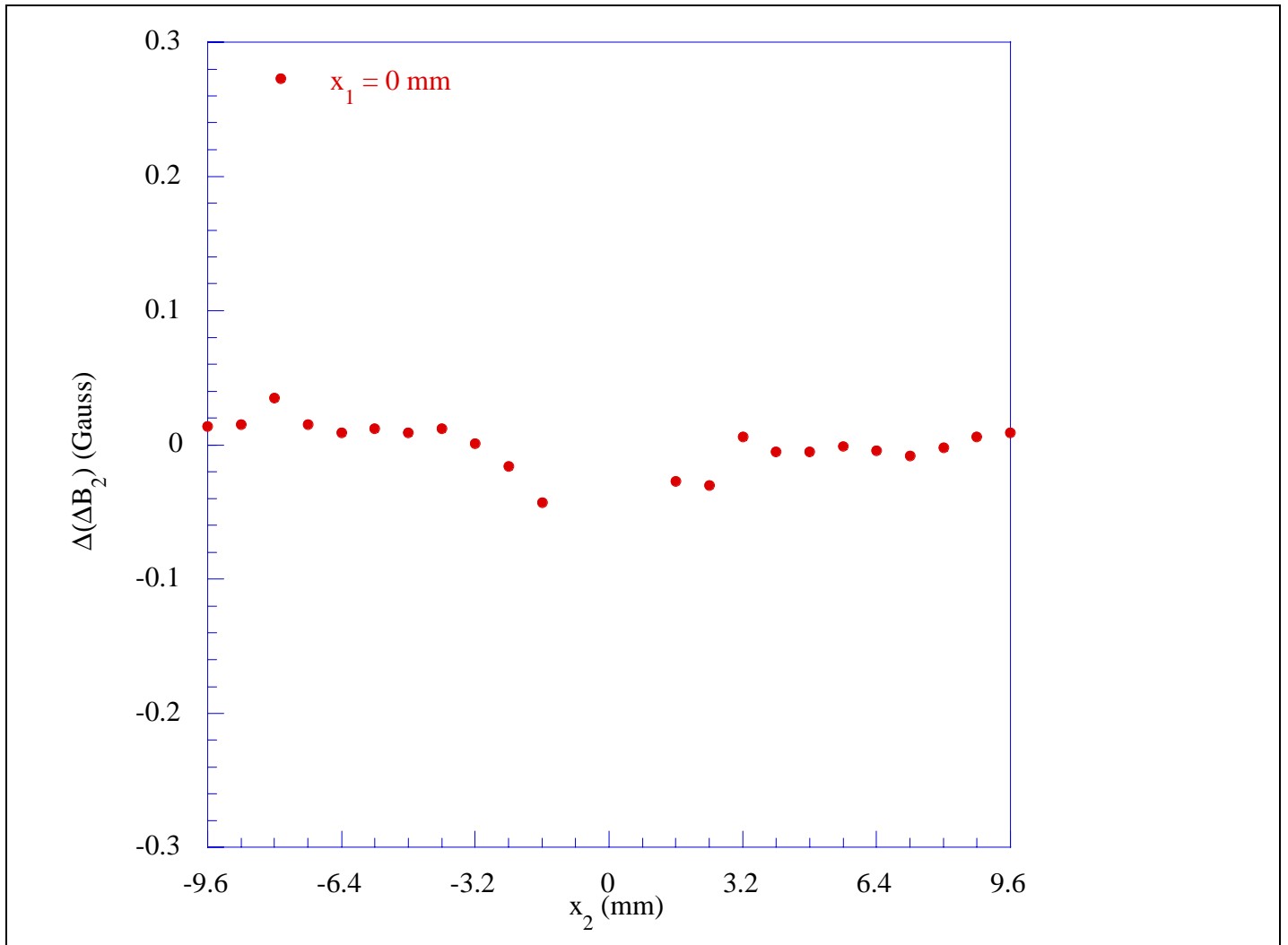

Figure 42. Difference between damaged (hole) and undamaged $\Delta B_{2}$ readings for terfenol-D FRP specimens at $\sigma_{1}=24 \mathrm{MPa}$ with hole edges positioned at $X_{2}=+1.6 \mathrm{~mm}$ and $-1.6 \mathrm{~mm}$. 


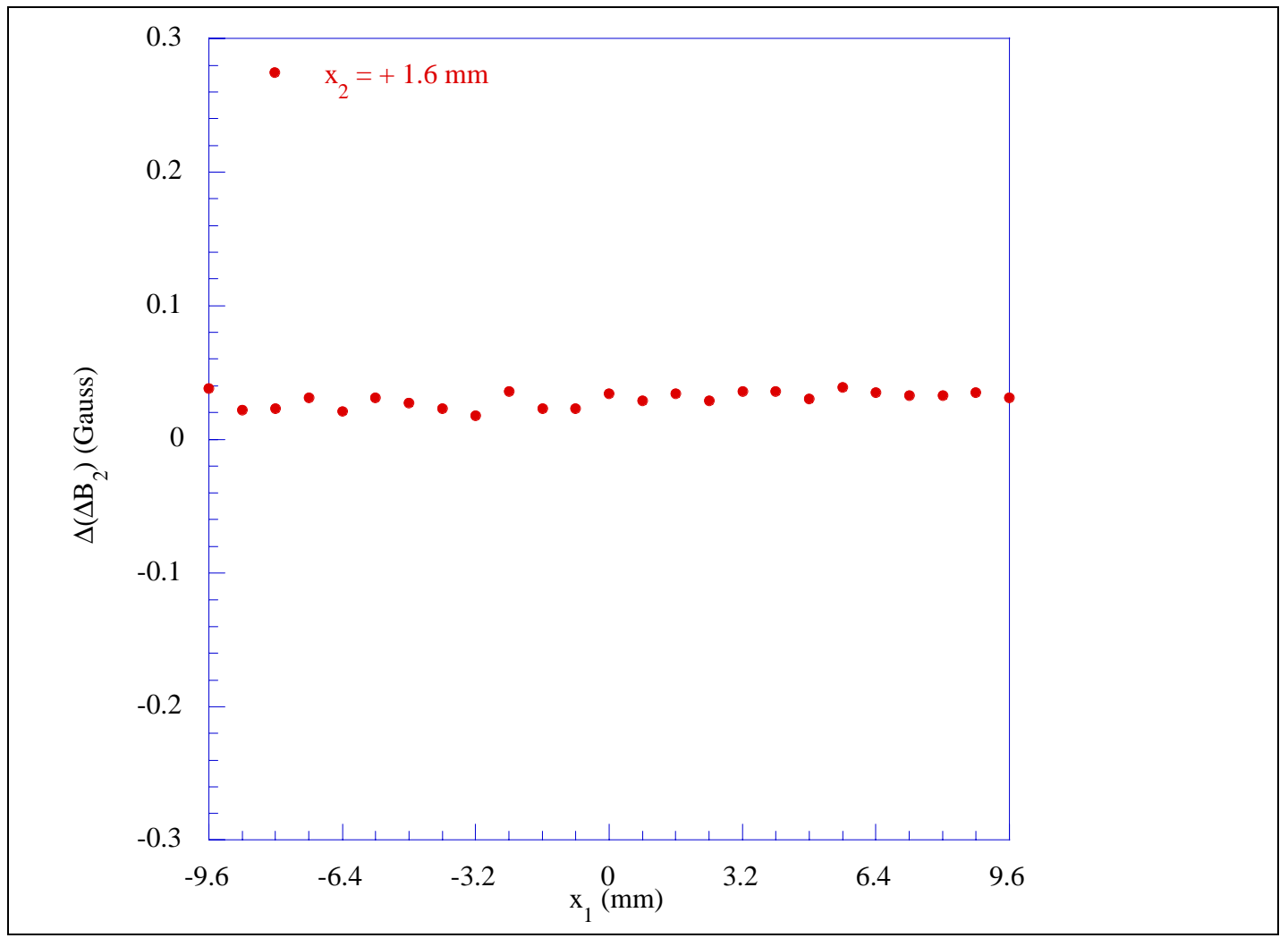

Figure 43. Difference between damaged (hole) and undamaged $\Delta B_{2}$ readings for terfenol-D FRP specimens at $\sigma_{1}=24 \mathrm{MPa}$ with hole edges positioned at $x_{2}=+1.6 \mathrm{~mm}$ and $-1.6 \mathrm{~mm}$.

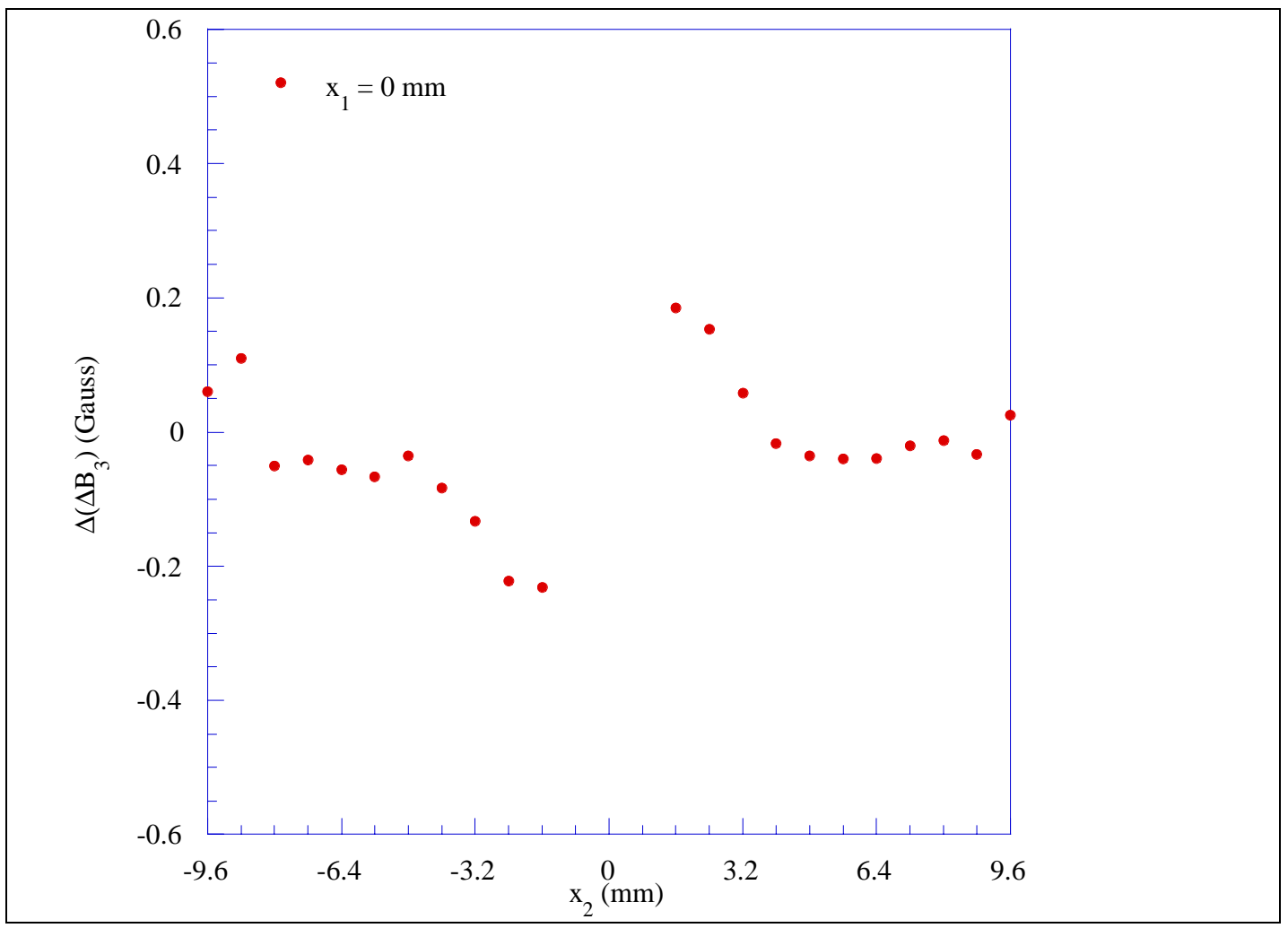

Figure 44. Difference between damaged (hole) and undamaged $\Delta B_{3}$ readings for terfenol-D FRP specimens at $\sigma_{1}=24 \mathrm{MPa}$ with hole edges positioned at $x_{2}=+1.6 \mathrm{~mm}$ and $-1.6 \mathrm{~mm}$. 


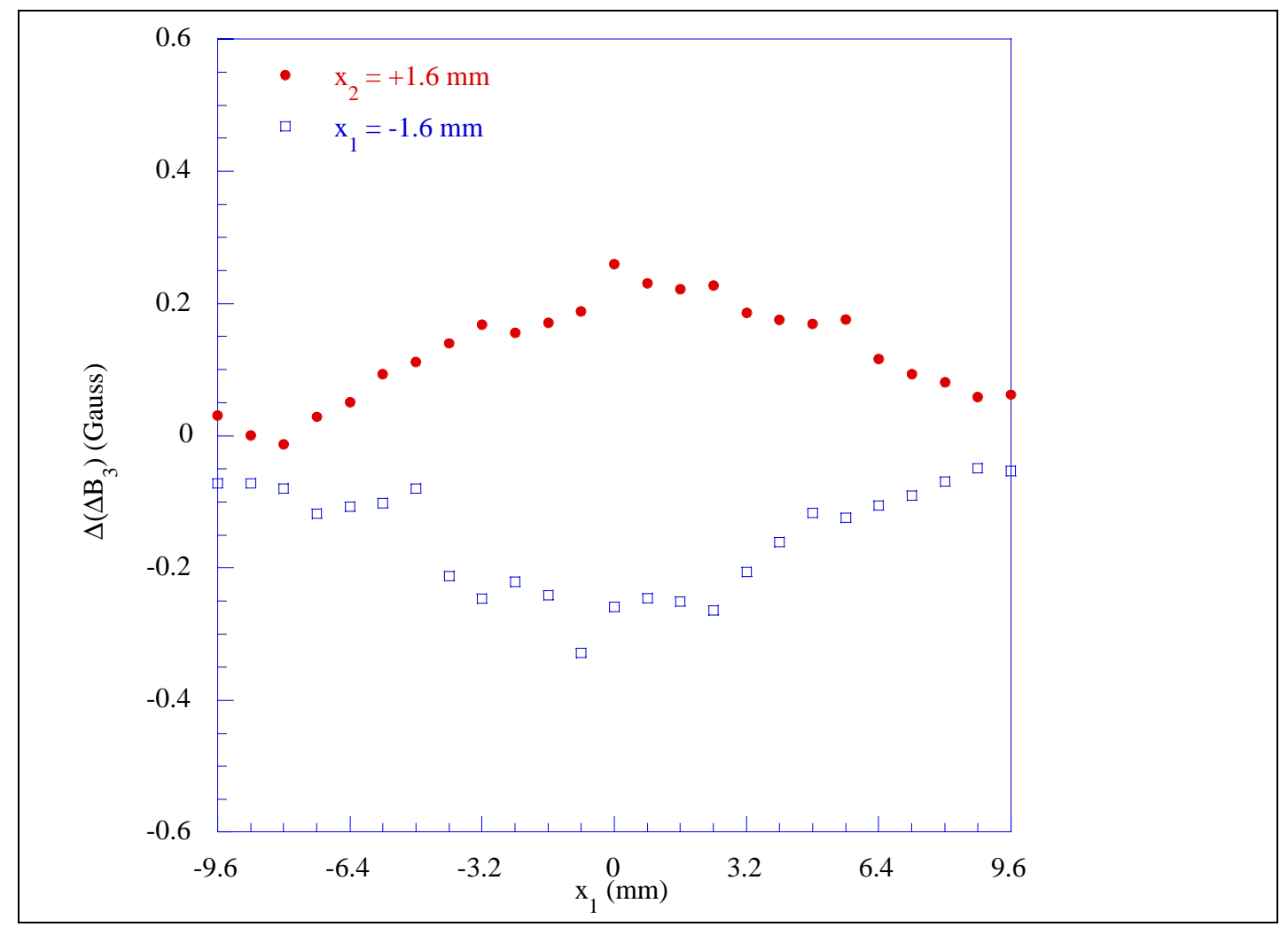

Figure 45. Difference between damaged (hole) and undamaged $\Delta B_{3}$ readings for terfenol-D FRP specimens at $\sigma_{1}=24 \mathrm{MPa}$ with hole edges positioned at $x_{2}=+1.6 \mathrm{~mm}$ and $-1.6 \mathrm{~mm}$.

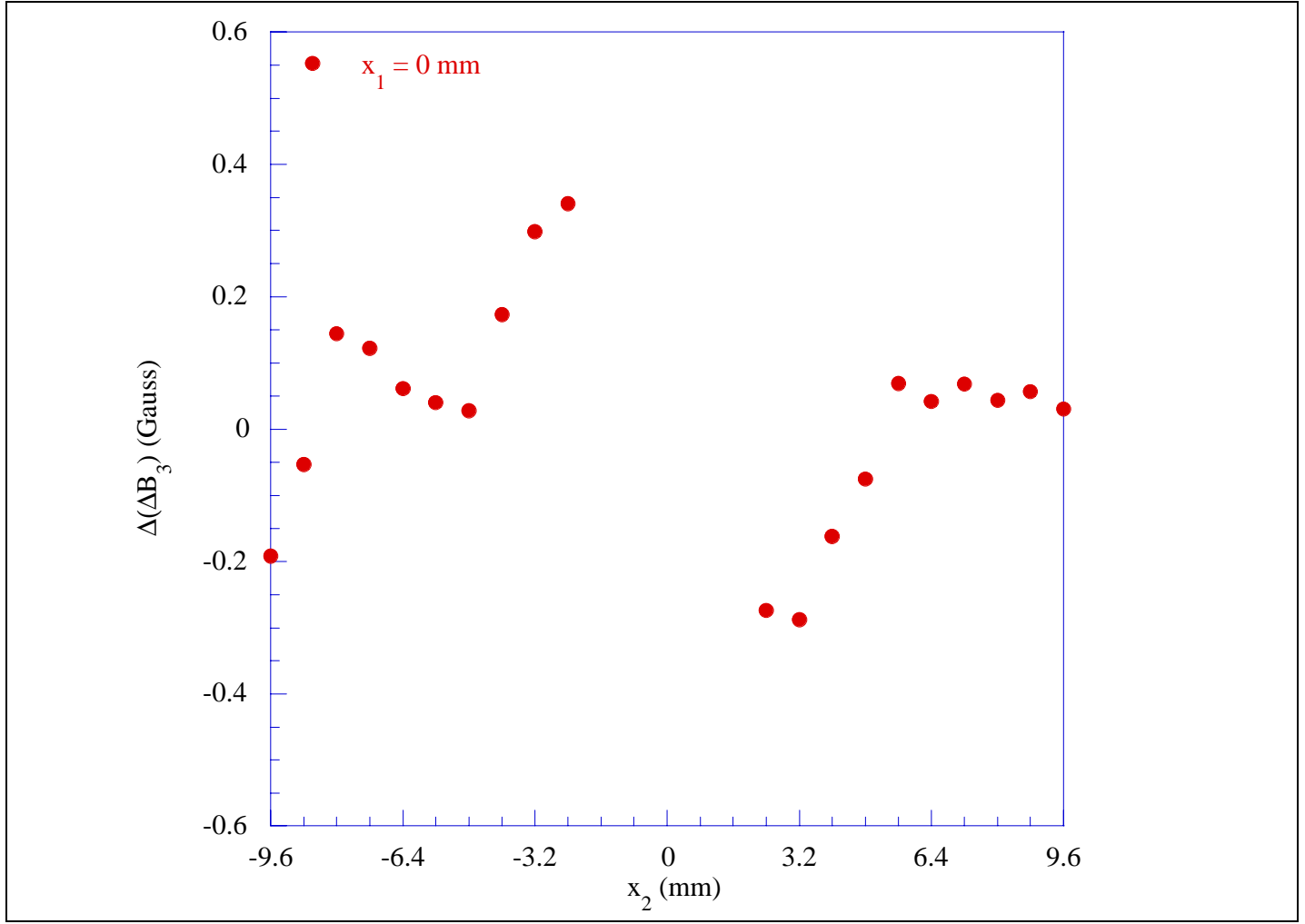

Figure 46. Difference between damaged (hole) and undamaged $\Delta B_{3}$ readings for terfenol-D FRP specimens at $\sigma_{1}=24 \mathrm{MPa}$ with hole edges positioned at $x_{2}=+2.4 \mathrm{~mm}$ and $-2.4 \mathrm{~mm}$. 


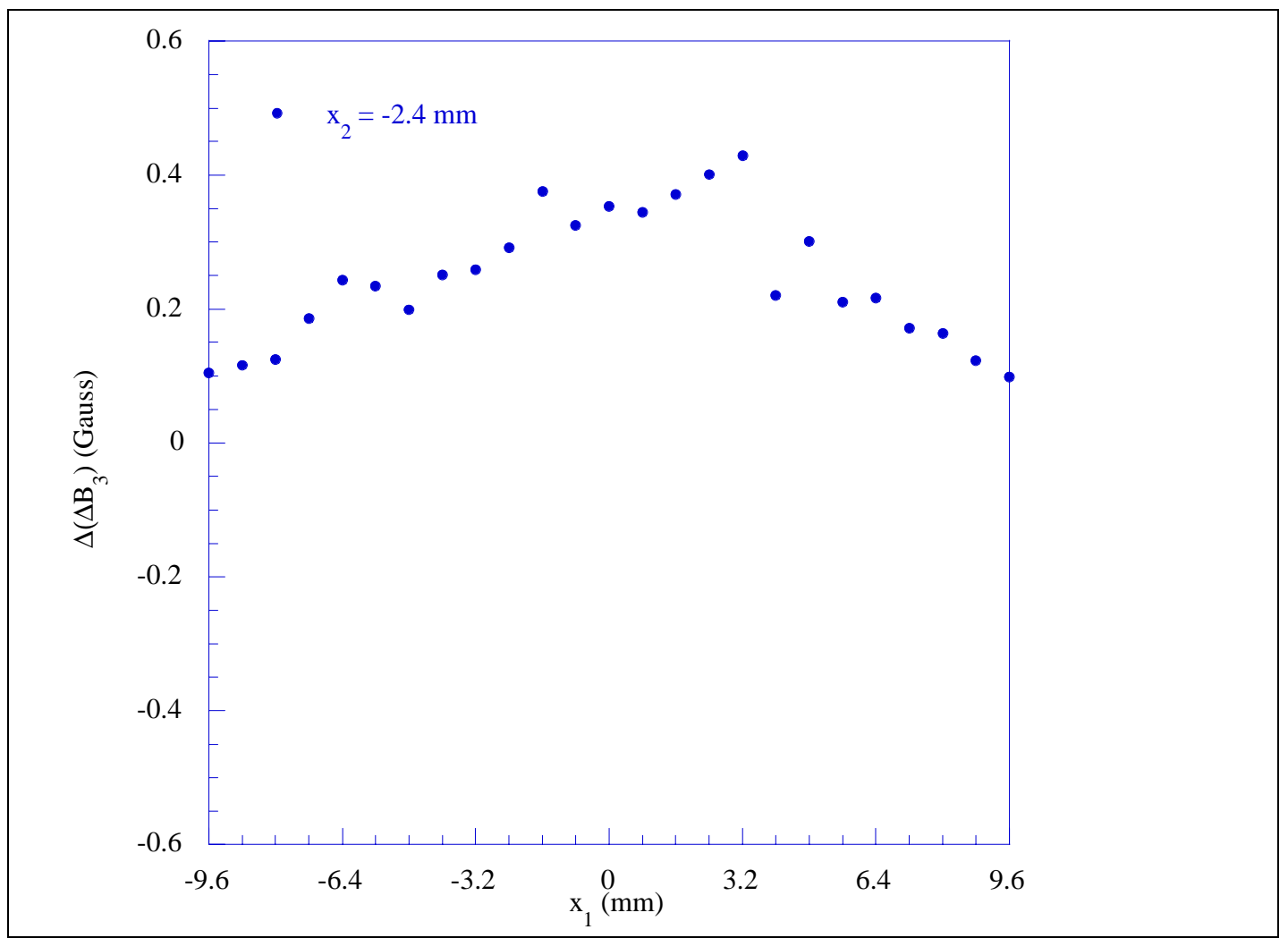

Figure 47. Difference between damaged (hole) and undamaged $\Delta B_{3}$ readings for terfenol-D FRP specimens at $\sigma_{1}=24 \mathrm{MPa}$ with hole edges positioned at $x_{2}=+2.4 \mathrm{~mm}$ and $-2.4 \mathrm{~mm}$.

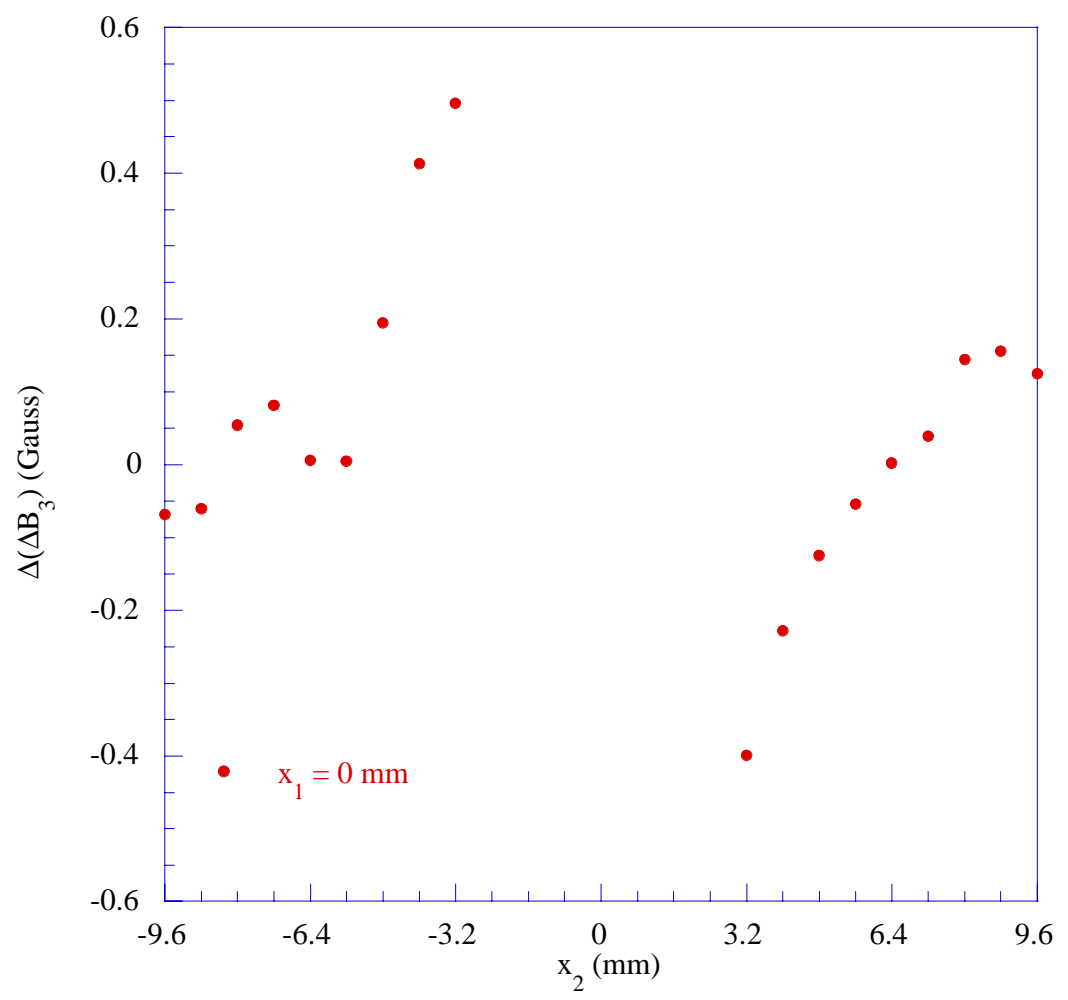

Figure 48. Difference between damaged (hole) and undamaged $\Delta B_{3}$ readings for terfenol-D FRP specimens at $\sigma_{1}=24 \mathrm{MPa}$ with hole edges positioned at $\mathrm{x}_{2}=+3.2 \mathrm{~mm}$ and $-3.2 \mathrm{~mm}$. 


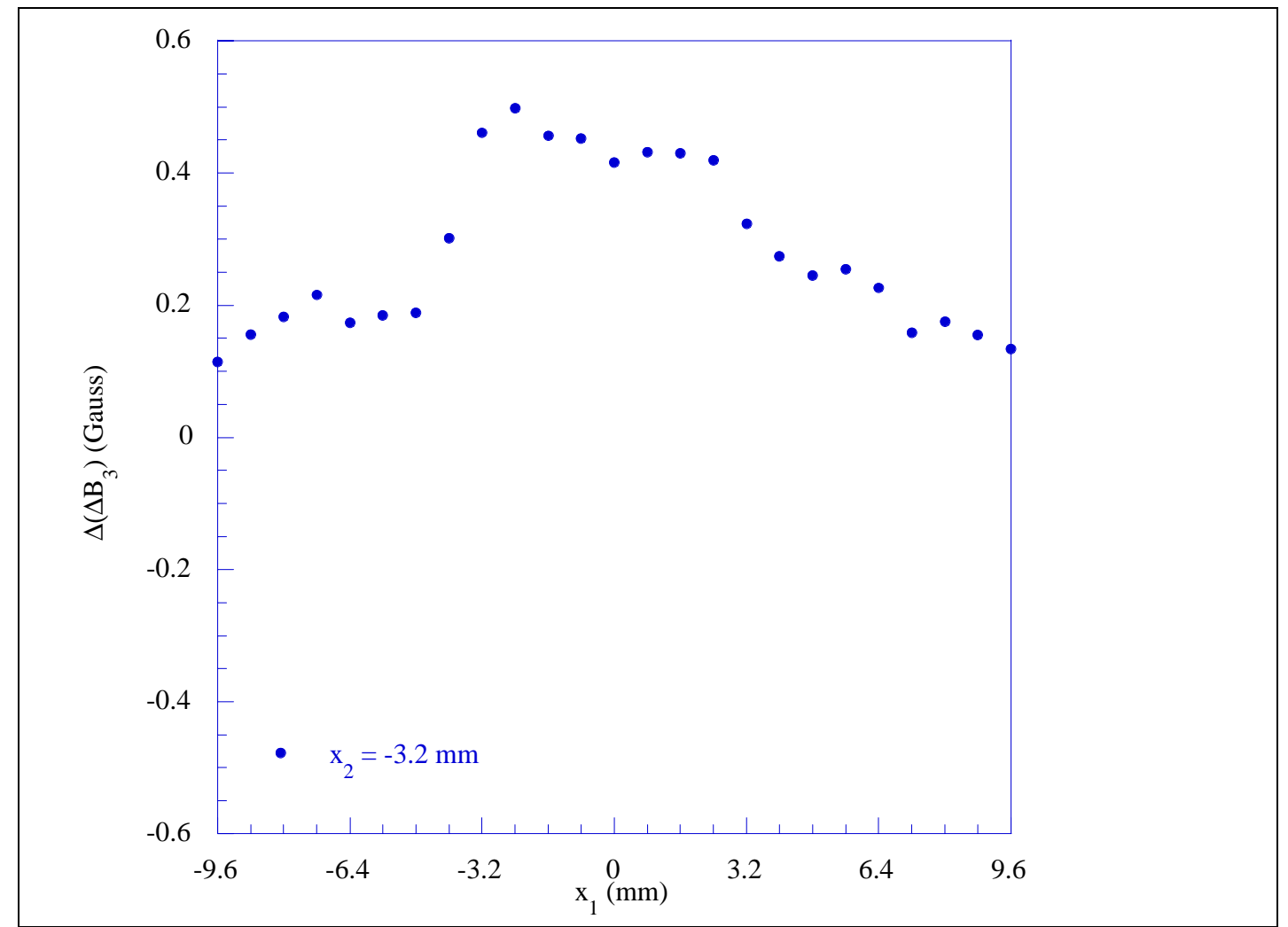

Figure 49. Difference between damaged (hole) and undamaged $\Delta B_{2}$ readings for terfenol-D FRP specimens at $\sigma_{1}=24 \mathrm{MPa}$ with hole edges positioned at $x_{2}=+3.2 \mathrm{~mm}$ and $-3.2 \mathrm{~mm}$. 


\section{Fabrication and Testing of Sputtered Composite Samples}

\section{Description of the Sputtering Process}

The high surface energy and specific gravity of terfenol-D particles (ball-milled to $-45 \mu \mathrm{m}$ ) cause the rare earth compound to coalesce into conglomerations of physically bonded particles and settle in the liquid resin before the matrix has cured. This tendency can inhibit uniform distribution of the particles onto the composite's reinforcement fibers. In an experiment that attempted to achieve more uniform dispersion of the magnetostrictive tagging particles, a process called cold sputtering was used to apply terfenol-D from a precut ingot disc (Etrema Products, Inc., Ames, IA) directly to two different types of glass-fiber fabrics.

In the process of sputtering, an energetic ion or neutral atom strikes the surface of a target and imparts momentum to the atoms over a range of a few nanometers (Goldstein et al. 1992). Some atoms receive enough energy to break bonds with neighboring atoms and separate from the target. The incident ions are produced using argon gas to obtain a clean, efficient, repeatable sputter medium. Permanent magnets are used to create a sputtering plasma ring and to deflect negative ions and electrons away from the substrate. The deposition rate is affected by several factors. Impurities in the bombarding gas, evolution of oxygen or water vapor, increases in specimen temperature, or distance between target and substrate can each be detrimental to the sputter coating process.

The sputtering apparatus was a DV-502A High-Vacuum Evaporator (Denton Vacuum, I nc., Moorestown, NJ ). The thermal evaporator contained a DSM-300A sputtering module attachment to allow for cold sputtering. The sputtering target was $2 \mathrm{in}$. in diameter and 1/16 in. in thickness. Prior to coating, a presputter pressure of approximately $3 \times 10^{-6}$ torr was obtained. In order to avoid contamination, the specimen and chamber were cleaned by glow discharge at $30 \mu$ torr Argon for 5 - 10 minutes, and the target surface was cleaned by bombardment at $7 \mu$ torr Argon and $280 \mathrm{~V}$. During the target cleaning, the flow of particles to the 
substrate was blocked by a shutter. Each side of the glass fiber substrate was coated for 60 minutes at $7 \mu$ torr Argon, $320-330 \mathrm{~V}$, and $0.75-0.85 \mathrm{~A}$.

The two fiber geometries used as the substrate for sputtering were an eight-satin harness weave and a unidirectional fiber. Sections of each sputtered sample were used to reinforce composite tensile specimens. Sections of the satin-weave fibers were analyzed using scanning electron microscopy (SEM) and energy dispersive spectroscopy, as outlined in the next section. Two sections were cut from the center of each sputtered sample to fit a $7 \times 0.75 \times 0.25 \mathrm{in}$. silicone tensile specimen mold. Two FRP specimens - one with each fiber geometry - were fabricated using an epoxy resin system comprising Shell EPON 828 and EpiCure 3140 (Miller-Stephenson, Inc., CT). Because the unidirectional fabric was thicker than the satin weave, four layers of the unidirectional and ten layers of the satin weave were used to manufacture composite samples of the same respective thickness.

\section{SEM Analysis of Sputtered Composite Samples}

The sputtered samples were manufactured in order to improve upon the distribution of terfenol-D particles in a tagged composite member. Figure 50 clearly illustrates some of the chief concerns about the introduction of powder directly into the resin. Some regions have little to no coverage because the particles tend to coalesce locally. The sputtered samples show promise for achieving a more uniform dispersion of the coating particles. The dark fibers in Figure 51 display a uniform coating with good evidence that the sputtered terfenol-D is able to penetrate the fibers thoroughly. 


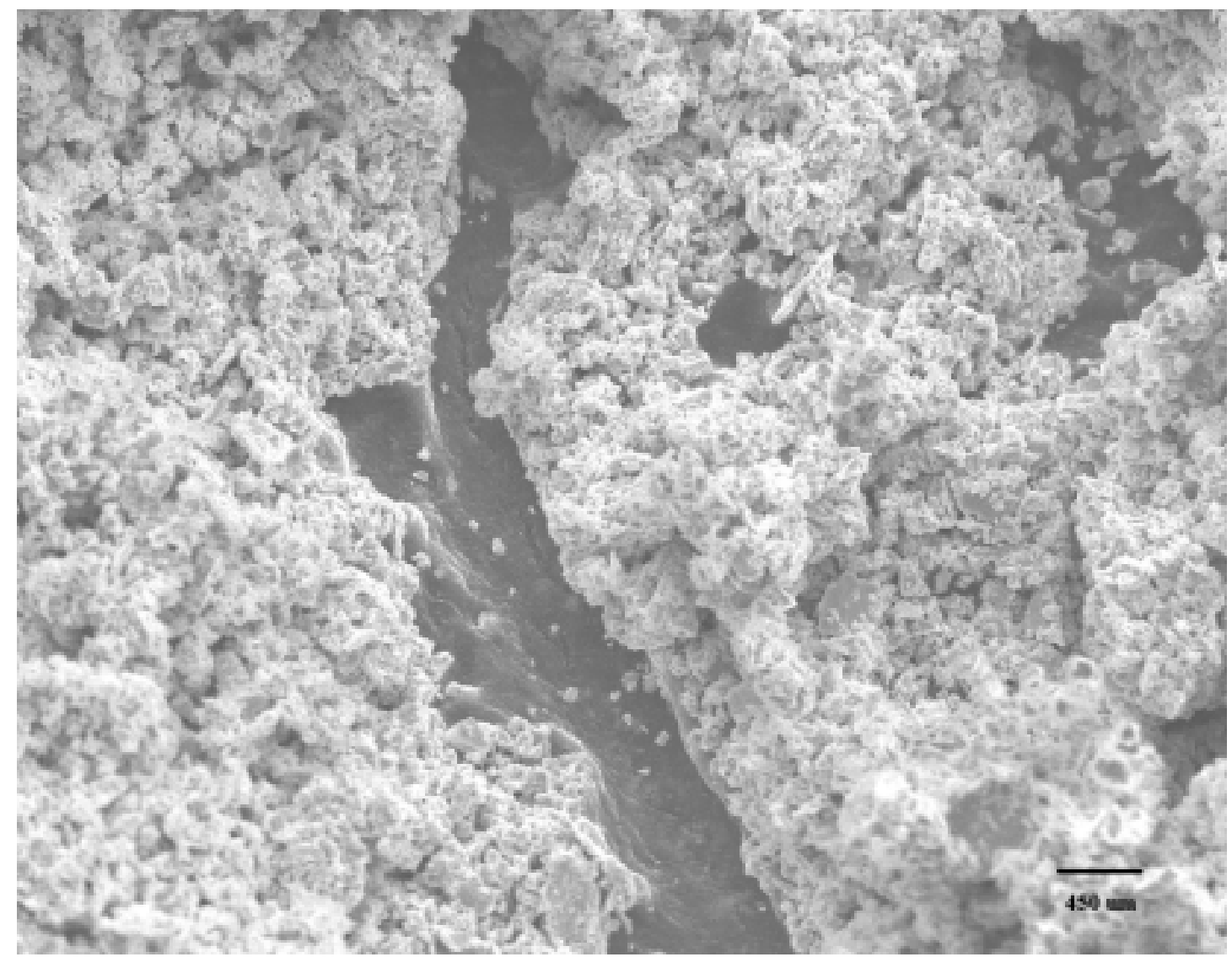

Figure 50. SEM image of terfenol-D powder particles, showing coalescence of individual particles and non-uniform distribution (magnification: 256X). 


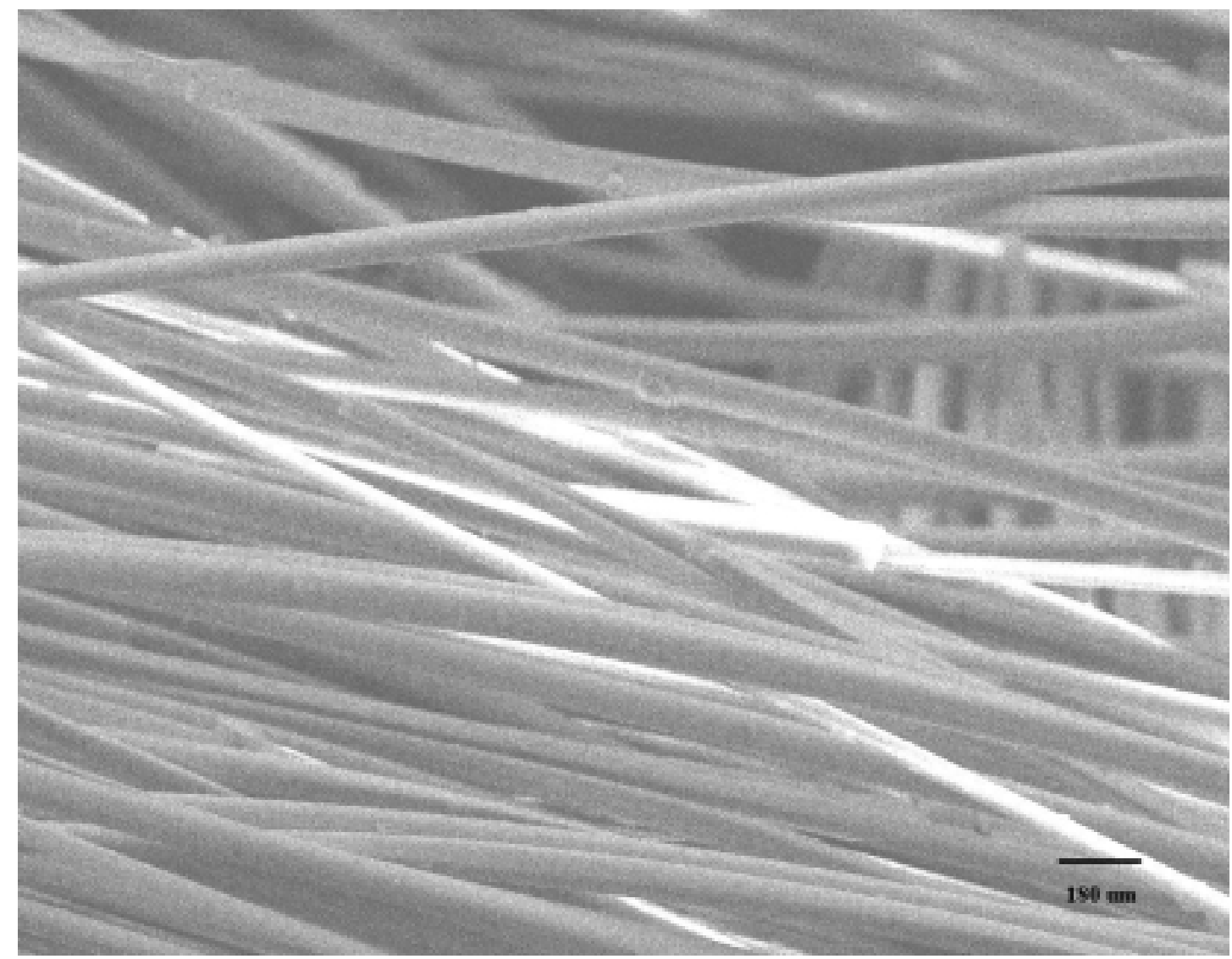

Figure 51. SEM image of sputtered terfenol-D on satin fiber weave, showing uniform distribution on the individual glass fibers throughout the thickness (magnification: $378 \mathrm{X}$ ).

However, there are several questions about the sputtering process that must be answered to determine its feasibility. In order to consider scaling the process to design scale it is necessary to verify the integrity of the sputtered layers of reinforcement fabric. Verification must include measurement as well as compositional analysis of the layer. The thickness $\mathrm{T}$ of the layer, in nanometers, can be estimated by the equation:

$$
T=\frac{C V t k}{10}
$$

where $\mathrm{C}$ is the plasma discharge in milliamperes, $\mathrm{V}$ the accelerating voltage in kilovolts, $\mathrm{t}$ the time in minutes, and $\mathrm{k}$ a constant depending on the bombarding gas and the target material. Special in situ devices can be used to calculate the coating thickness during coating. The most accurate methods include direct calculation using transmission electron microscopy, x-ray absorption and emission, or gravimetric analysis after coating. 


\section{Energy Dispersive Spectroscopy Analysis}

Compositional analysis of the terfenol-D coating is necessary to determine the effect of different densities and thermal behavior on deposition rate. Energy dispersive spectroscopy (EDS) was performed on a section of a terfenol-D ingot, the hammer-milled powder, and the sputtered fiber weave to ensure that the sputter coating was of the same chemical composition as the other forms. Although quantitative x-ray analysis is difficult with the EDS technique due to the introduction of spectral artifacts, the spectra of the three forms were very similar. The results indicate that the sputtering method is feasible for production of uniformly coated impregnated fibers under laboratory conditions.

These specimens were tested according to the Control Protocol with annealing in the transverse direction. The results are shown for the unidirectional and satin weave composite samples in Figures 52 and 53, respectively. Figure 52 indicates a lower signal, probably because the unidirectional reinforcement contained less terfenol-D than the satin weave reinforcement. Note that the loading/unloading response shown in Figure 53 is flipped in comparison to all other zorro curves shown in this report.

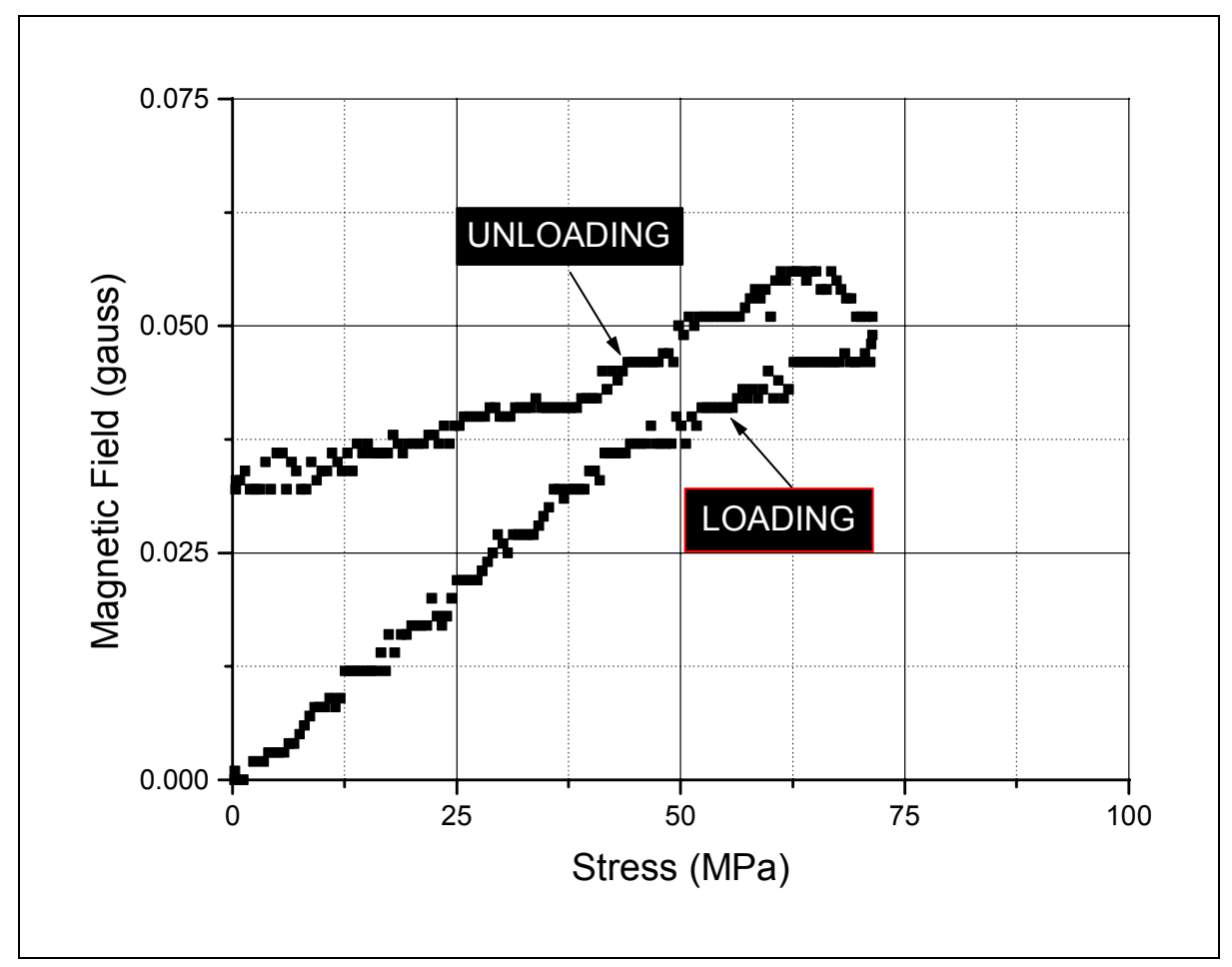

Figure 52. Loading and unloading of annealed sputtered unidirectional sample. 


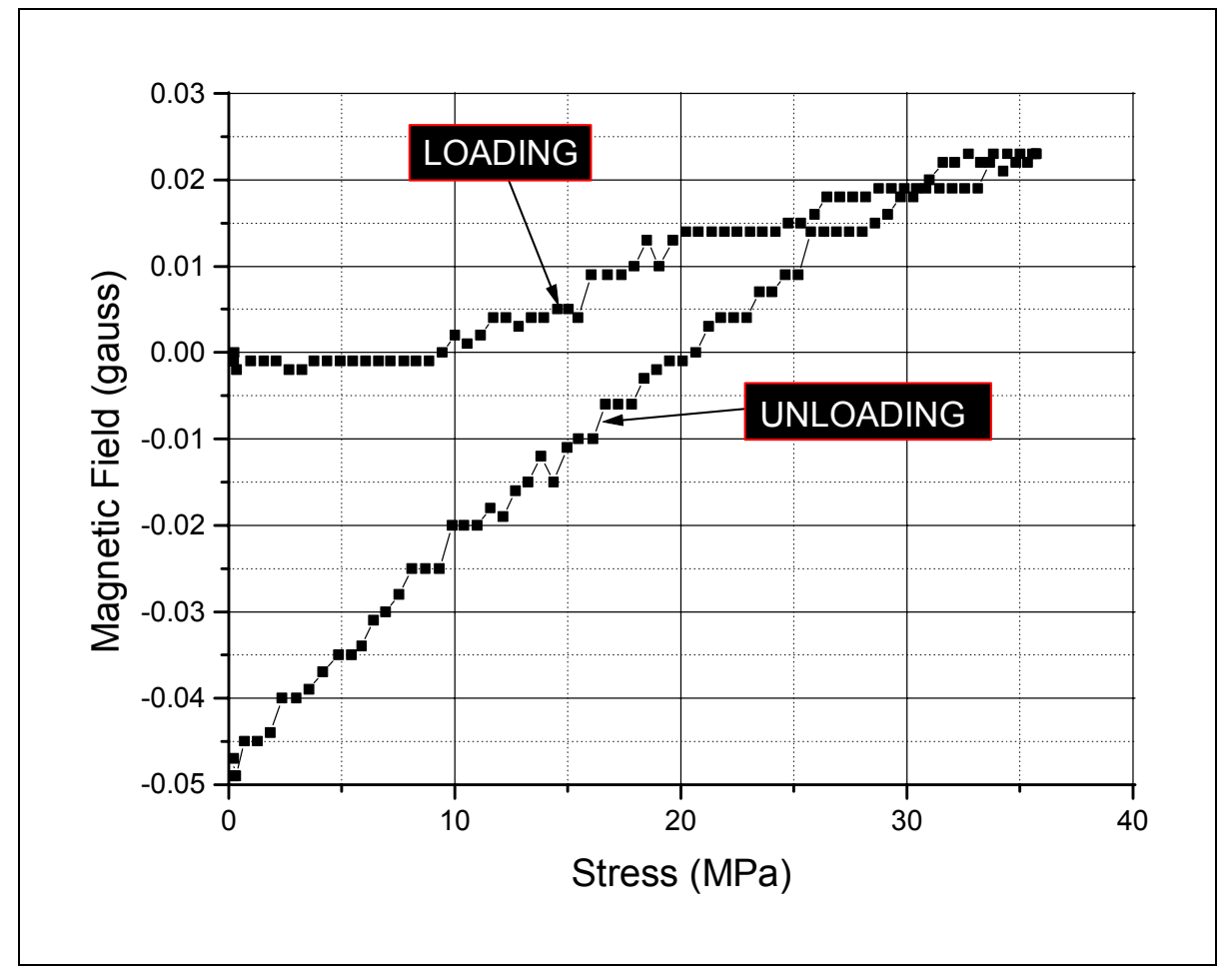

Figure 53. Loading and unloading of annealed sputtered weave sample. 


\section{Study of Magnetic Domain Structure in Terfenol-D Particles}

The domain structure of terfenol-D particles has a significant effect on the magnetomechanical response of the tagged composite member. To learn more about the domain structure of the terfenol-D particles used in this research a magnetic force microscope (MFM) was used.

The effect of magnetic annealing and particle size on the domain structure is of particular interest but they have not been fully characterized. However, some initial images of the domain structure of both large and small terfenol-D particles have been recorded. Figure 54 shows an MFM image of a polished section of an unreinforced (i.e., neat resin) 2.24 volume percent large terfenol-D specimen that was annealed during cure. The left image shows the topography of the specimen and the right image shows the magnetic force gradient data. Both images are of an area on the specimen measuring $50 \times 50 \mu \mathrm{m}$. Several particles of varying size can be seen in this image and there is a clear difference between particles and matrix in both the topography and gradient images. The domain structure of a particle does not seem to vary significantly with particle size. Also it is important to note that the domains are much smaller than the particles themselves. Figure 55 shows a close-up view of the boxed particle in Figure 54. In this close-up image it can be seen that the particle contains about 70 - 80 domains. Assuming spherical geometries for the particle and the domains, the average domain size in this case is roughly $0.3 \mu \mathrm{m}$. 


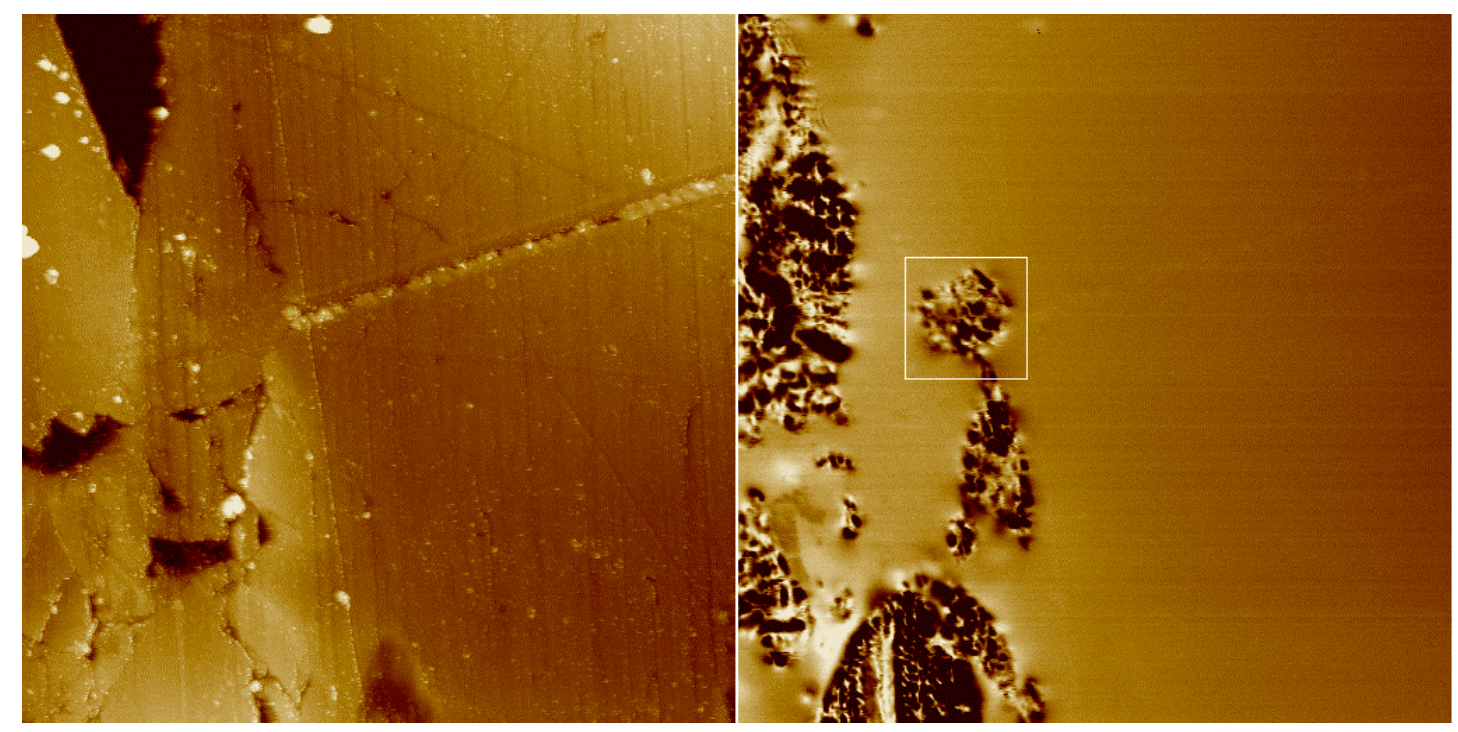

Height range: $500 \mathrm{~nm}$

Phase range: 4 degrees

50 by 50 microns

Figure 54. MFM topography and phase images of embedded terfenol-D particles from hammermilled powder.

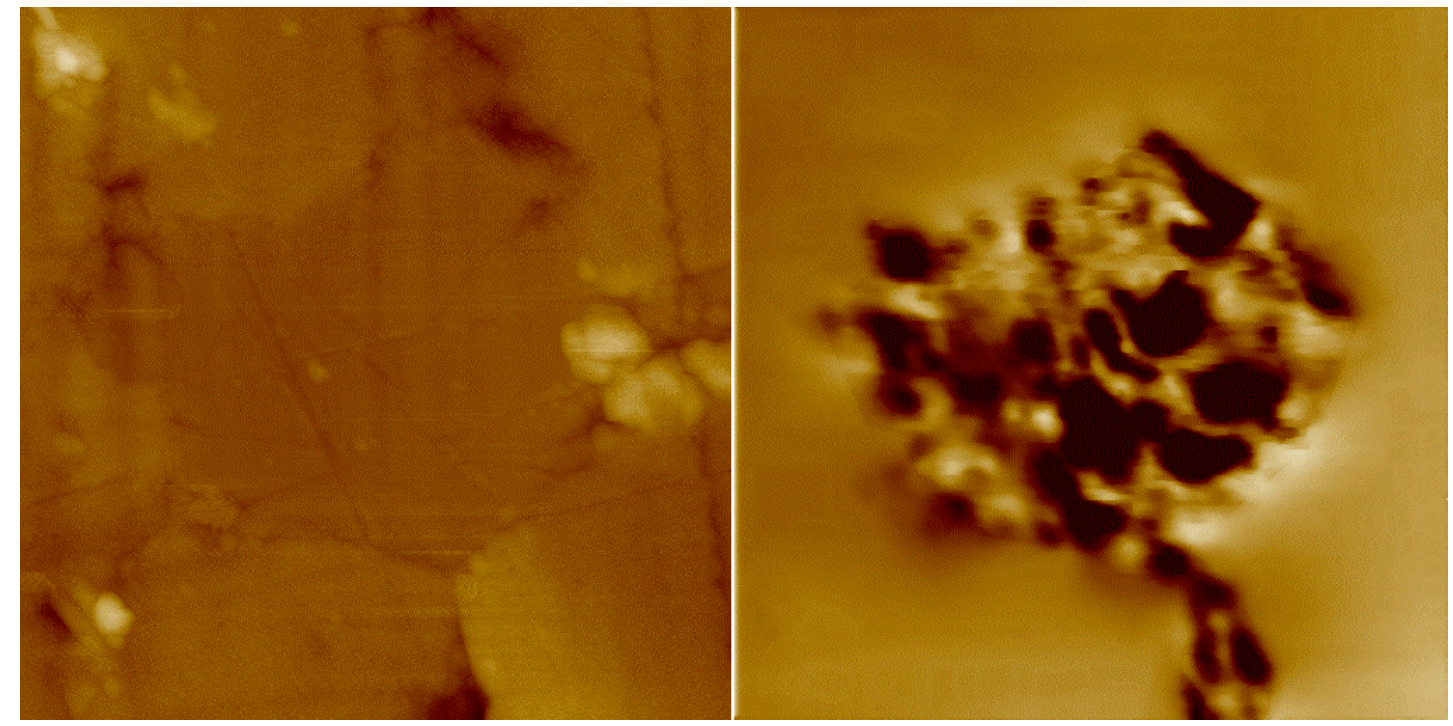

Height range: $500 \mathrm{~nm}$

Phase range: 4 degrees

10 by 10 microns

Figure 55. Close-up MFM topography and phase images of the 7 micron terfenol-D particle boxed in white in Figure 54. 
An MFM image of an unreinforced specimen with 2.24 volume percent terfenol-D from the ball-milled $(<10 \mu \mathrm{m})$ powder is shown in Figure 56. As before, the topography is on the left and the magnetic gradient data are on the right, and both images measure $50 \times 50 \mu \mathrm{m}$. In comparing the images of large $(<45 \mu \mathrm{m})$ and small ( $<10 \mu \mathrm{m}$ ball-milled) terfenol-D particles, a definite difference in domain structure can be seen. The new particles are magnetically different from the matrix but have little or no interior domain structure. This lack of interior structure could be an indication that oxidation occurred during the ball-milling of the small partides. However, more investigation is needed before validated conclusions can be drawn.

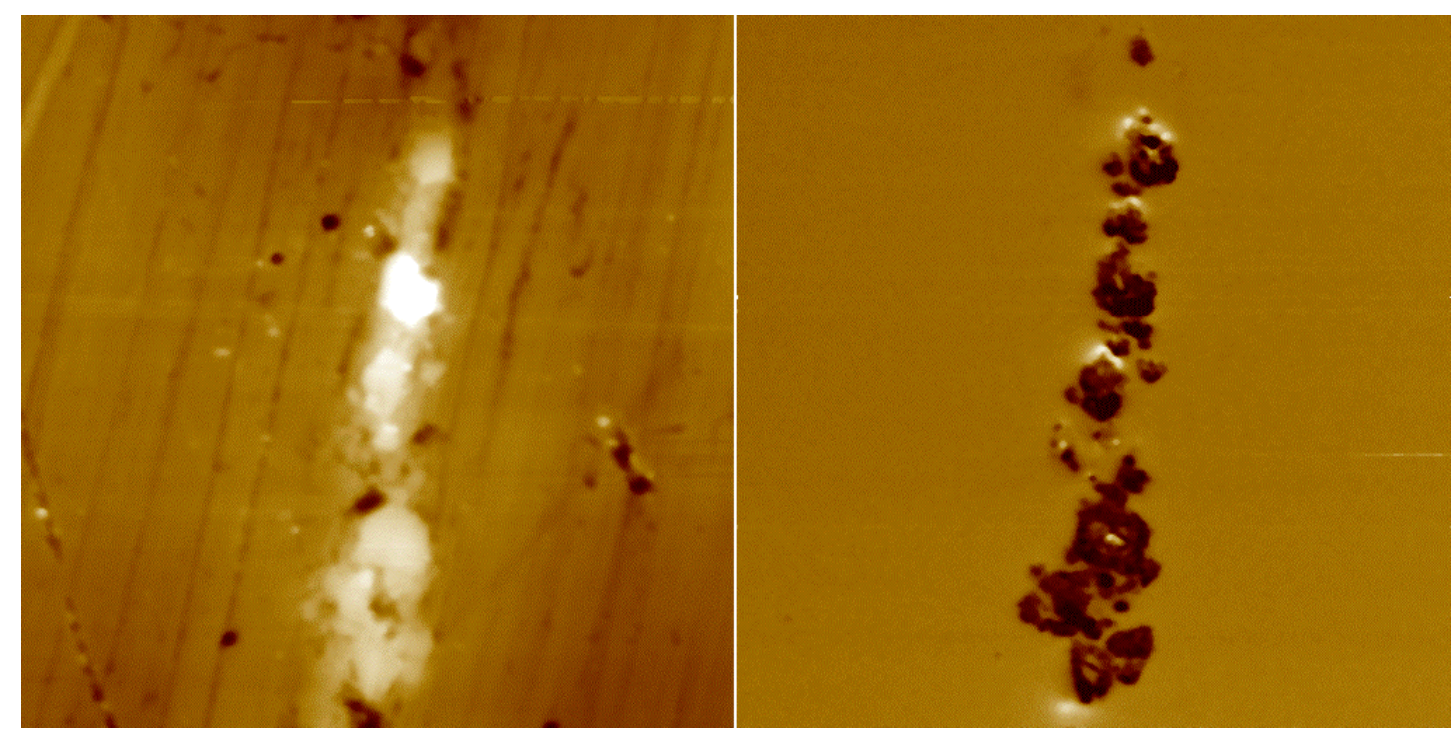

Height range: $600 \mathrm{~nm}$

Phase range: 4 degrees

50 by 50 microns

Figure 56. MFM topography and phase images of embedded terfenol-D particles from ball-milled powder. 


\section{Conclusions and Recommendations}

The studies documented in this report address theoretical and applied aspects of tagging FRP composites with magnetostrictive particles to enable the monitoring of loads, strains, and damage in structural members using portable, readily available nondestructive monitoring equipment. After theoretical models were developed, various types of magnetostrictive-tagged resin matrices - both unreinforced and glass-fiber reinforced - were designed, fabricated, tested, and analyzed in laboratory experiments.

\section{Conclusions}

It is concluded that magnetostrictive-tagged FRP composites behave in general accordance with a CERL-developed semi-quantitative, mesoscale phenomenological model, based on modified Heisenberg-Weiss theory, that relates the macroscopic behavior of a magnetostrictive material with nanoscale magnetic domain theory. Based on the results of a series of laboratory experiments and tests, the following specific conclusions are offered:

1. When subjected to various stresses, tagged samples of both unreinforced resin and glass-fiber reinforced resin produced magnetic flux signals that could be re corded with a standard Hall-effect probe. Good repeatability in the data was observed in test series where the magnetic domains of the tagged material were realigned (or annealed) between stress cycles. Samples that were not magnetically anneal ed between stressing progressively lost signal strength, reaching a steady state after about 32 stress cycles. This signal reduction is attributed to irreversible domain wall motion, but reannealing the sample returned the signal to its first-cycle response.

2. The application of a low-strength magnetic field during cure of the tagged matrix material aids in the suspension of terfenol-D particles in the resin, aligning them into 'chains' al ong the direction of the applied field. This 'chaining' process improves the signal strength of a cured specimen by a factor of 18 over an unchained specimen of the same composition.

3. In tension tests of tagged samples, flux readings taken in the transverse direction were consistently much stronger than readings taken in the axial direction. This 
difference was so significant and consistent that, in the majority of the stress tests, magnetic flux readings were made only in the transverse direction.

4. Comparison of the current flexural test results with previously published results for axial loading (Trovillion et al. 1999) indicates that the coefficient for the annealed bending specimen was 28.4 percent higher than that for the unannealed axial specimen. Comparison of data for the annealed bending specimen and the axial tension specimen showed that the bending specimen coefficient was about one-third that of the axial specimen. This difference may be due to the nonuniform stress distribution through the thickness of the bending specimen or to differences between the two specimens in terms of fiber geometry and architecture.

5. The results of damage detection experiments show that the magnetic flux signatures of a preloaded magnetostrictive-tagged composite specimen can be used to detect damage introduced to that specimen.

6. An initial demonstration of sputtering technology for application of terfenol-D partides directly to the reinforcement substrate, as opposed to mixing them into the resin matrix, indicates that sputtering may improve the uniformity of partide distribution, and this benefit could in theory improve the magnetostrictive properties of the tagged composite. SEM analysis provides some evidence of better uniformity of partide distribution using the sputtering technique.

7. Initial microstructural analysis of tagged composite specimens indicates that domain structure does not seem to vary with particle size; domain size is very small in comparison to particlesize; and some factor - possibly oxidation during particle milling - can impede the formation or retention of interior magnetic domains.

Taken collectively, the results of these studies indicate that magnetostrictivetagged composites have great potential for application in self-monitoring structural technologies in the field.

\section{Recommendations}

This research to date has focused on laboratory-based testing, observation, and analysis. The work reported here produced several results that require study before large-scale fabrication and field demonstration can be accomplished. To provide key data needed before developing Army-critical applications, support of the following research activities is recommended: 
1. A follow-on study is needed to determine why magnetic flux signals are so much stronger when read transverse to the axis of tension loading compared to the axial direction.

2. Further investigation is required to determine the feasibility of fabricating sputtered magnetostrictive composite members economically at a design and production scale, as well as to determine the performance benefits sputter-application may offer.

3. More detailed microstructural studies are needed to develop an adequate technical understanding of the magnetostrictive properties of terfenol-D, especially with respect to the effects of corrosion products, contamination, and other factors on magnetic domain behavior. 


\section{References}

Adly, A.A., I .D. Mayergoyz, and A. Bergqvist, "Preisach Modeling of Magnetostrictive Hysteresis," J ournal of Applied Physics, Vol 69, No. 8, 1991, pp 5777-5779.

Bagdassaryan, G.E., and E.A. Danoyan, "Mathematical Modeling of the Vibrations of Two-Layer Magnetostrictive Plates," Izv. AN SSSR. Mekhanika Tverdogo Tela, Vol 27, No. 3, 1992, pp 8794.

Benbouzid, M., Reyne, G., and Meunier, G., “N onlinear Finite Element Modeling of Giant Magnetostriction," IEEE Transactions on Magnetics, Vol Mag-29, No. 6, 1993, pp 2467-2469.

Bergqvist, A., and G. Engdahl, "A Stress-Dependent Magnetic Preisach Hysteresis M odel," IEEE Transactions on Magnetics, Vol Mag-27, No. 6, 1991, pp 4796-4798.

Berman, J ustin B., Robert F. Quattrone, Aaron Averbuch, F. Lalande, H. Cudney, V. Raju, and G.L. Cohen, "Piezoelectric Patch Sensors for Structural Integrity Monitoring of CompositeU pgraded Masonry and Concrete Structures," Construction Engineering Research Laboratory (CERL, Champaign, IL) Technical Report (TR) 99/072, ADA369164, August 1999.

Brouwers, F., "Magnetostrictive Tagging of Composite Materials for Structural Health Monitoring," Master's Thesis, University of Illinois (Urbana-Champaign), Department of Aeronautical and Astronautical Engineering, October 1998.

Chen, Chih-Wen, "Magnetism and Metallurgy of Soft Magnetic Materials," Dover Publications, Inc., New York, 1986.

Clark, A.E., J .D. Verhoven, O.D. McMasters, and E.D. Gibson, "Magnetostriction in Twinned [112] Crystals of Tb.27Dy.73Fe2," IEEE Transactions on Magnetics, Vol Mag-22, 1986, pp 973-975.

Clark, G.E., B.K. Tanner, and H.T. Savage, Philosophical Magazines B46, 331, (1982).

du Tremolet de Lacheisserie, Etienne "Magnetostriction: Theory and Applications of Magnetoelasticity," CRC Press, Boca Raton, FL, 1993.

Engdahl, G., and L. Kvarnsjö, “A Time Dependent Radially Resolved Simulation Model of 'Giant' Magnetostrictive Materials," Mechanical Modeling of New Electromagnetic Materials, Elsevier Science Publishers, ed. R.K.T. Hsieh, 1990, pp 131-138.

Engdahl, G., and L. Svensson, "Simulation of the Magnetostrictive Performance of Terfenol-D in Mechanical Devices,"J ournal of Applied Physics, Vol 63, No. 8, 1988, pp 3924-3926.

Engdahl, Goran (editor), "Handbook of Giant Magnetostrictive Materials," (Chapter 1), Academic Press, San Diego, CA 92101-4495 (1999). 
Etrema Products, Inc., 2500 North Loop Drive, Ames, IA 50010.

Feickert, C.A., J . Berman, J . Kamphaus, and J . Trovillion, "A semiquantitative analytic model for the magnetic response of a Terfenol-D impregnated composite specimen under tension,"J ournal of Applied Physics, 88:4265-4268 (2000).

Giurgiutiu, V., F. J ichi, and C.A. Rogers, "Design, Fabrication and Testing of a MagnetostrictiveTagged Fiber-Reinforced Composite Specimen," Report \#USC-ME-LAMSS-99-106, J une 30, 1999.

Goldstein, J oseph et al., "Scanning Electron Microscopy and X-ray Microanalysis." Plenum, New York, 1992.

Heisenberg, W., Zeitschrift fur Physik, 49, 619 (1928).

Hommema, J .A., "Magnetomechanical Behavior of Terfenol-D Particulate Composites," Master's Thesis, University of Illinois (Urbana-Champaign), Department of Theoretical and Applied Mechanics, October 1999.

Hudson, J ., S.C. Busbridge, and A.R. Piercy, "Magnetomechanical Coupling and Elastic Moduli of Polymer-Bonded TERFENOL Composites,"J ournal of Applied Physics, 83:7255-7257 (1998).

J iles, D.C., and J .B. Thoelke, "Modeling of the Combined Effects of Stress and Anisotropy on the Magnetostriction of Tb.3Dy.7Fe2," IEEE Transactions on Magnetics, Vol Mag-27, No. 6, 1991, pp 5352-5354.

J olly, M.R., J .D. Carlson, B.C. Muñoz, and T.A. Bullions, "The Magnetoviscoelastic Response of Elastomer Composites Consisting of Ferrous Particles Embedded in a Polymer Matrix,"J ournal of Intelligent Material Systems and Structures, 7:613-622 (1996).

Kittel, C., "I ntroduction to Solid State Physics," (5th edition), J ohn Wiley and Sons, Inc., New York (1976).

Koon, N., and C.M. Williams, J . Phys., Paris, 40, C5, 194, (1978).

Kvarnsjö, L., and G. Engdahl, "Nonlinear 2-D Transient Modeling of Terfenol-D Rods," IEEE Transactions on Magnetics, Vol Mag-27, No. 6, 1991, pp 5349-5351.

$\mathrm{Li}$, J in, "Magnetostrictive Tagging of Composite Materials and Their Magnetomachanical Behavior," U niversity of Illinois (Urbana-Champaign), Department of Aeronautical and Astronautical Engineering, 2000.

Mitrovic, M., M. Robert, and G. Carman, "Magnetostrictive Composite Material Systems Analytical/Experimental," Proceedings of the American Society for Composites Tenth Technical Conference, Technomic Publishing Co., Inc., Lancaster, PA, 1995, pp 85-94.

Pinkerton, F.E., T.W. Capehart, J .F. Herbst, E.G. Brewer, and C.B. Murphy, “Magnetostrictive SmFe/metal Composites," Applied Physics Letters, 70:2601-2603 (1997). 
Quattrone R., J .B. Berman, and S.R. White, "Self-Monitoring Structures Containing Magnetostrictive Composite," Proceedings, 21st Army Science Conference, J une 15-17,1998, Norfolk, VA, pp 21-26.

Quattrone, R.F., J .B. Berman, Z. Chaudhry, V. Giurgiutiu, C.A. Rogers, and S.R. White, "I nvestigation of Active Tagged Composites for Army Infrastructure Applications," CERL TR 98/022, ADA344919, April 1998.

Roberts, M., M. Mitrovic, and G. Carman, "Nonlinear Behavior of Coupled Magnetostrictive Material Systems Analytical/Experimental," Proceedings of the SPIE Smart Materials Conference, San Diego, CA, 1995.

Rogers, C.A., S.-W. Zhou, and Z. Chaudhry, "An Embedded Particle Tagging Technique for In-Field Non-Destructive Evaluation," CE RL TR, November 1994.

Rogers, C.A., S.-W. Zhou, and Z. Chaudhry, "Concepts for Active Tagging of Reinforced Composites for In-Process and In-Field Non-Destructive Evaluation," CE RL TR, March 1995.

Sandlund, L., M. Fahlander, T. Cedell, A.E. Clark, J .B. Restoff, and M. Wun-Fogle, "Magnetostriction, Elastic Moduli, and Coupling Factors of Composite TERFEN OL-D,"J ournal of Applied Physics, 75:5656-5658 (1994).

Teter, J .P., K.B. Hathaway, and A.E. Clark, J ournal of Applied Physics, 79 (8), 6213, 15 April 1996.

Trovillion J .C., J . Kamphaus, R. Quattrone, and J .B. Berman, "Structural Integrity Monitoring Using Smart Magnetostrictive Composites," Proceedings of I nternational Composite Exposition and Symposi um, May 1999, Cincinnati, OH.

Weiss, P., J our. Phys., 6, 667 (1907).

White S.R., and F. Brouwers, "Smart Materials for Infrastructure Applications", CERL TR, CE RL Contract \#DACA88-97-K-0001, J une 24, 1998.

White S.R., and R.G. Albers, "Magnetostrictive Tagging of Composite Materials for Structural Health Monitoring," CERL TR, February 1996.

White, S.R., "Magnetostrictive Tagging of Composites for Health Monitoring," Proceedings, 1999 International Composites Expo, Cincinnati, OH, May 10-12, 1999, pp 22E 1 - 22 E6.

White, S.R., R.G. Albers, and R.F. Quattrone, "Tagging of Composite Materials with Terfenol-D Magnetostrictive Particles for Structural Health Monitoring," ASME 1996 Mechanics and Materials Summer Meeting, J une 12-14, 1996, J ohns Hopkins University, Baltimore, MD. 


\section{CERL Distribution}

Chief of Engineers

ATTN: CEHEC-IM-LH (2)

ATTN: CEMP-ED

Engineer Research and Development Center (Libraries)

ATTN: ERDC, Vicksburg, MS

ATTN: Cold Regions Research, Hanover, NH

ATTN: Topographic Engineering Center, Alexandria, VA

Defense Tech Info Center 22304

ATTN: DTIC-O

7 


\section{REPORT DOCUMENTATION PAGE}

Form Approved

OMB No. 0704-0188

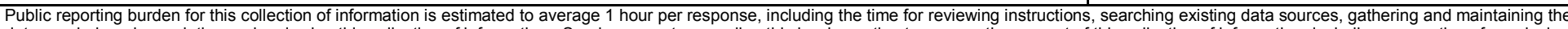

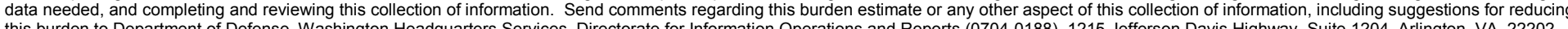

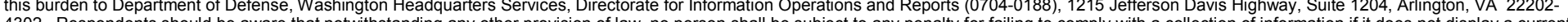

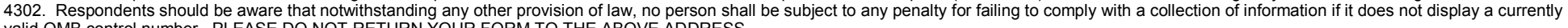
valid OMB control number. PLEASE DO NOT RETURN YOUR FORM TO THE ABOVE ADDRESS.

\begin{tabular}{|l|l|l}
\hline 1. REPORT DATE (DD-MM-YYYY) & 2. REPORT TYPE & 3. DATES COVERED (From - To)
\end{tabular} $12-2000$

Final

4. TITLE AND SUBTITLE

Investigation of Terfenol-D for Magnetostrictive Tagging of Fiber-Reinforced Polymer

Composites 5a. CONTRACT NUMBER

5b. GRANT NUMBER

5c. PROGRAM ELEMENT NUMBER

6. AUTHOR(S)

Robert F. Quattrone, Justin B. Berman, Jonathan C. Trovillion, Carl A. Feickert, Jason M.

Kamphaus, Scott R. White, Victor Giurgiutiu, and Gordon L. Cohen

5d. PROJECT NUMBER

611102AT23

5e. TASK NUMBER

5f. WORK UNIT NUMBER

FM-E00

8. PERFORMING ORGANIZATION REPORT

NUMBER

ERDC/CERL TR-00-46

U.S. Army Engineer Research and Development Center (ERDC)

Construction Engineering Research Laboratory (CERL)

P.O. Box 9005

Champaign, IL 61826-9005

9. SPONSORING / MONITORING AGENCY NAME(S) AND ADDRESS(ES)

HQUSACE

ATTN: CEMP-ED, Rm 3114

$441 \mathrm{G}$ Street, NW

Washington, DC 20314-1000

NUMBER(S)

\section{DISTRIBUTION / AVAILABILITY STATEMENT}

Approved for public release; distribution is unlimited.

\section{SUPPLEMENTARY NOTES}

Copies are available from the National Technical Information Service, 5285 Port Royal Road, Springfield, VA 22161.

\section{ABSTRACT}

The U.S. Army maintains an aging inventory of over 143,000 buildings representing $\$ 4.6$ billion in high priority, unfinanced maintenance and repair. Many of these structures fail to meet modern building codes and require structural upgrades. The objective of this research was to develop a cost-effective magnetostrictive fiber-reinforced polymer (FRP) composite material that can be fabricated into structural elements capable of generating data about their own structural condition, and which can be monitored and assessed by measuring its electromagnetic flux over time. The magnetrostrictive material selected for these tagging experiments was terfenol-D.

The studies documented in this report address theoretical and applied aspects of tagging FRP composites with magnetostrictive particles to enable the monitoring of loads, strains, and damage in structural members using portable, readily available nondestructive monitoring equipment. After theoretical models were developed, various types of magnetostrictive-tagged resin matrices - both unreinforced and glass-fiber reinforced - were designed, fabricated, tested, and analyzed in laboratory experiments. Taken collectively, the results of these studies indicate that magnetostrictive-tagged composites have great potential for application in self-monitoring structural technologies in the field.

\section{SUBJECT TERMS}

fiber-reinforced polymer (FRP)

composite materials

\begin{tabular}{|l|l|l|}
\hline \multicolumn{2}{|l|}{ 16. SECURITY CLASSIFICATION OF: } & 17. \\
\hline $\begin{array}{c}\text { a. REPORT } \\
\text { Unclassified }\end{array}$ & $\begin{array}{c}\text { b. ABSTRACT } \\
\text { Unclassified }\end{array}$ & $\begin{array}{c}\text { c. THIS PAGE } \\
\text { Unclassified }\end{array}$ \\
\end{tabular}

smart materials

magnetostriction
terfenol-D

\begin{tabular}{|c|c|c|}
\hline $\begin{array}{c}\text { 17. LIMITATION } \\
\text { OF ABSTRACT }\end{array}$ & $\begin{array}{c}\text { 18. NUMBER } \\
\text { OF PAGES }\end{array}$ & $\begin{array}{c}\text { 19a. NAME OF RESPONSIBLE PERSON } \\
\text { Robert F. Quattrone }\end{array}$ \\
\cline { 3 - 3 } SAR & 80 & $\begin{array}{c}\text { 19b. TELEPHONE NUMBER (in- } \\
\text { clude area code) }\end{array}$ \\
\hline
\end{tabular}

Standard Form 298 (Rev. 8-98) Prescribed by ANSI Std. 239.18 